\title{
TORIC TOPOLOGY OF THE COMPLEX GRASSMANN MANIFOLDS
}

\author{
VICTOR M. BUCHSTABER AND SVJETLANA TERZIĆ
}

\begin{abstract}
The family of the complex Grassmann manifolds $G_{n, k}$ with the canonical action of the torus $T^{n}=\mathbb{T}^{n}$ and the analogue of the moment map $\mu: G_{n, k} \rightarrow \Delta_{n, k}$ for the hypersimplex $\Delta_{n, k}$, is well known. In this paper we study the structure of the orbit space $G_{n, k} / T^{n}$ by developing the methods of toric geometry and toric topology. We use a subdivision of $G_{n, k}$ into the strata $W_{\sigma}$. Relying on this subdivision we determine all regular and singular points of the moment map $\mu$, introduce the notion of the admissible polytopes $P_{\sigma}$ such that $\mu\left(W_{\sigma}\right)=\stackrel{\circ}{P}_{\sigma}$ and the notion of the spaces of parameters $F_{\sigma}$, which together describe $W_{\sigma} / T^{n}$ as the product $\stackrel{\circ}{P}_{\sigma} \times F_{\sigma}$. To find the appropriate topology for the set $\cup_{\sigma} \stackrel{\circ}{P_{\sigma}} \times F_{\sigma}$ we introduce also the notions of the universal space of parameters $\tilde{\mathcal{F}}$ and the virtual spaces of parameters $\tilde{F}_{\sigma} \subset \tilde{\mathcal{F}}$ such that there exist the projections $\tilde{F}_{\sigma} \rightarrow F_{\sigma}$. Having this in mind, we propose a method for the description of the orbit space $G_{n, k} / T^{n}$. The existence of the action of the symmetric group $S_{n}$ on $G_{n, k}$ simplifies the application of this method. In our previous paper we proved that the orbit space $G_{4,2} / T^{4}$, which is defined by the canonical $T^{4}$-action of complexity 1 , is homeomorphic to $\partial \Delta_{4,2} * \mathbb{C} P^{1}$. We prove in this paper that the orbit space $G_{5,2} / T^{5}$, which is defined by the canonical $T^{5}$-action of complexity 2 , is homotopy equivalent to the space which is obtained by attaching the disc $D^{8}$ to the space $\Sigma^{4} \mathbb{R} P^{2}$ by the generator of the group $\pi_{7}\left(\Sigma^{4} \mathbb{R} P^{2}\right)=\mathbb{Z}_{4}$. In particular, $\left(G_{5,2} / G_{4,2}\right) / T^{5}$ is homotopy equivalent to $\partial \Delta_{5,2} * \mathbb{C} P^{2}$.

The methods and the results of this paper are very important for the construction of the theory of $(2 l, q)$-manifolds we have been recently developing, and which is concerned with manifolds $M^{2 l}$ with an effective action of the torus $T^{q}, q \leq l$ and an analogue of the moment map $\mu: M^{2 l} \rightarrow P^{q}$, where $P^{q}$ is a $q$-dimensional convex polytope.
\end{abstract}

\section{CONTENTS}

1. Introduction

2. The canonical action of $T^{n}$ on $G_{n, k}$

2.1. Plücker coordinates

2.2. Moment map

2.3. Hypersimplex

2.4. Admissible polytopes and strata

2.5. $\left(\mathbb{C}^{*}\right)^{n}$-action on $G_{n, k}$

2.6. The space of parameters of the main stratum

2.7. Action of the symmetric group $S_{n}$

2.8. The $T^{n}$-action on the Thom spaces

3. Orbit spaces of the strata

3.1. Orbits of $\left(\mathbb{C}^{*}\right)^{k}$-action on $\mathbb{C}^{n}$

3.2. On singular and regular values of the moment map

2000 Mathematics Subject Classification. 57S25, 57N65, 53D20, 14M25, 52B11, $14 \mathrm{~B} 05$.

Key words and phrases. Grassmann manifold, Thom spaces, torus action, orbit spaces, spaces of parameters. 
4. The canonical action of $\left(\mathbb{C}^{*}\right)^{5}$ on $G_{5,2} \quad 18$

4.1. $\left(\mathbb{C}^{*}\right)^{5}$-orbits and their admissible polytopes 18

4.2. Admissible polytopes in the chart $M_{12}$. 19

4.3. The admissible polytopes and the strata for $G_{5,2}$. 21

4.4. The combinatorial approach to admissible polytopes. 21

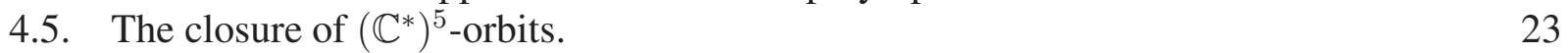

4.6. The strata in the chart $M_{12}$, their spaces of parameters and orbit spaces 24

4.7. The facets of the admissible polytopes $\quad 25$

5. The idea of the universal space of parameters $\quad 26$

6. The spaces $\bar{F}_{\sigma, 12}$ for the strata $W_{\sigma}$ in the chart $M_{12} \quad 27$

7. The universal space of parameters $\tilde{\mathcal{F}} \quad 28$

7.1. The closure $\mathcal{F}$ of $F$ in $\mathbb{C} P^{1} \times \mathbb{C} P^{1} \times \mathbb{C} P^{1} \quad 28$

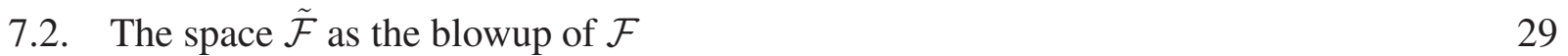

8. The transition automorphisms for the spaces of parameters of the strata 31

8.1. The transtion automorphisms for the space of parameters $F$ of the main stratum 32

8.2. The transition automorphisms for the spaces of parameters of other non one-orbit strata

9. The proof of Theorem 5.1

9.1. The virtual spaces of parameters in the chart $M_{12}$

9.2. The projection from $\tilde{F}_{\sigma, i j}$ to $F_{\sigma}$.

10. An algebraic manifold in $\left(\mathbb{C} P^{1}\right)^{5}$ which realizes $\tilde{\mathcal{F}} \quad 41$

11. The orbit space $G_{5,2} / T^{5}\left(\mathbb{C}^{1}\right)^{5}$ which realizes $\mathcal{F}_{4}$

11.1. Summary 44

11.2. Description of the orbit space $G_{5,2} / T^{5} \quad 44$

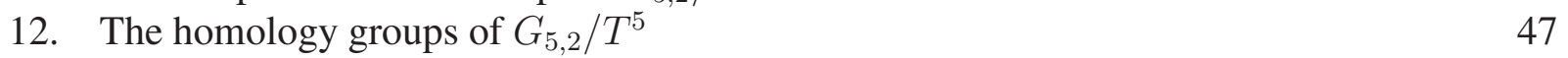

12.1. $V_{1}$ and its homology groups $\quad 47$

12.2. $V_{2}$ and its homology groups 4

12.3. The homology groups for $V_{3}=G_{5,2} / T^{5}$.

References

\section{INTRODUCTION}

There is a canonical action of the algebraic torus $\left(\mathbb{C}^{*}\right)^{n}$ on the algebraic manifold $G_{n, k}$ of all $k$-dimensional complex vector subspaces $L$ in the $n$-dimensional complex vector space $\mathbb{C}^{n}$ and, consequently, an action of the compact torus $T^{n}=\mathbb{T}^{n} \subset\left(\mathbb{C}^{*}\right)^{n}$. The problems of the description of these actions are well known and the interest in these problems is motivated by the classic and modern problems of algebraic geometry, algebraic and equivariant topology, symplectic geometry and enumerative combinatorics, see [11], [12], [13], [20], [19]. In the focus of our study is an action of the torus $T^{n}$ on $G_{n, k}$. The manifolds $G_{n, k}$ and $G_{n, n-k}$ are equivariantly diffeomorphic, so we can assume that $k \leq\left[\frac{n}{2}\right]$. There is an analogue of the moment map [12] for $G_{n, k}$ whose image is the hypersimplex $\Delta_{n, k}$. In the case when $k=1$ we obtain the complex projective space $\mathbb{C} P^{n-1}$, which is a fundamental example of a toric manifold and $\Delta_{n, 1}$ is a simplex. For $k \geq 2$ the combinatorics of the polytope $\Delta_{n, k}$ does not determine the structure of the orbit space $G_{n, k} / T^{n}$. 
Using the classical Plücker coordinates for $k$-dimensional subspace $L$ in $\mathbb{C}^{n}$, we introduce $\left(\mathbb{C}^{*}\right)^{n}$ equivariant subdivision of the manifold $G_{n, k}$ by the strata $W_{\sigma}$, where $\sigma$ runs through the set of the so-called admissible subsets of the set $\{1, \ldots, m\}$, where $m=\left(\begin{array}{l}n \\ k\end{array}\right)$. The disjoint union of all $W_{\sigma} / T^{n}$ gives a subdivision of the orbit space $G_{n, k} / T^{n}$. We want to emphasize that the strata we introduce coincide with the known strata introduced, although by different methods, in the paper of Gel'fand-Serganova [12]. The image of the moment map, when restricted to the stratum $W_{\sigma}$ is the interior of the admissible polytope $P_{\sigma}$, which is the convex hull of some subset of vertices for $\Delta_{n, k}$. We prove that all points from $W_{\sigma}$ have the same stabilizer $\mathbb{C}_{\sigma}^{*}$ and describe the singular and regular points for the moment map $\mu: G_{n, k} \rightarrow \Delta_{n, k}$. The orbit space $F_{\sigma}=W_{\sigma} /\left(\mathbb{C}^{*}\right)^{n}$ is an algebraic manifold which we call the space of parameters for $W_{\sigma}$. We consider the set $E$ which is defined as a disjoint union of the spaces $\stackrel{\circ}{P_{\sigma}} \times F_{\sigma}$, where $\sigma$ runs through all admissible sets. The canonical projections $W_{\sigma} \rightarrow \stackrel{\circ}{P_{\sigma}}$ and $W_{\sigma} \rightarrow F_{\sigma}$ define the homeomorphism $W_{\sigma} / T^{n} \rightarrow \stackrel{\circ}{P}_{\sigma} \times F_{\sigma}$ and we obtain the canonical bijection of the sets $E \rightarrow G_{n, k} / T^{n}$.

In our approach the set $E$ is the key object for the description of the structure of the orbit space $G_{n, k} / T^{n}$ and the key problem here is to introduce the topology on $E$ in terms of $P_{\sigma}$ and $F_{\sigma}$ such that the bijection $E \rightarrow G_{n, k} / T^{n}$ becomes a homeomorphism. In the realization of this approach we essentially had to develop the methods of toric topology [5], [6]. In the case of a quasitoric manifold $M^{2 n}$, the orbit space $M^{2 n} / T^{n}$ is homeomorphic to the polytope $P^{n}$, which is the image of the moment map. Thus, the orbit space $M^{2 n} / T^{n}$ is determined by the combinatorial structure of the polytope $P^{n}$. In the case of Grassmann manifolds, the points from the orbit space $G_{n, k} / T^{n}$ are determined by the two coordinates, one from $P_{\sigma}$ and the other from $F_{\sigma}$.

In order to equip the set $E$ with an appropriate topology we introduce the new notions: the universal space of parameters $\tilde{\mathcal{F}}$, which is an algebraic variety and, for any admissible set $\sigma$, the virtual space of parameters $\tilde{F}_{\sigma}$, such that there is the embedding $\tilde{F}_{\sigma} \subset \tilde{\mathcal{F}}$ and the projection $\tilde{F}_{\sigma} \rightarrow F_{\sigma}$. We also introduce the set $\mathcal{P}$ which is a formal union of the admissible polytopes $P_{\sigma}$ and define the topology on $\mathcal{P}$ using the fact that the moment map $G_{n, k} / T^{n} \rightarrow \Delta_{n, k}$ decomposes as $G_{n, k} / T^{n} \rightarrow \mathcal{P} \rightarrow \Delta_{n, k}$. The set $\mathcal{E}$ which is a union of all spaces $\stackrel{\circ}{P_{\sigma}} \times \tilde{F}_{\sigma}$ inherits the topology from the canonical embedding into the space $\mathcal{P} \times \tilde{\mathcal{F}}$. This yields a surjective mapping $H: \mathcal{E} \rightarrow E$, which defines a topology on $E$.

We want to note that Kapranov in [15] considered the Chow quotient $G_{n, k} / /\left(\mathbb{C}^{*}\right)^{n}$ which is an algebraic variety and proved that it can be identified with certain compactification of the space of parameters $F$ of the main stratum. Moreover, Kapranov proved that the Chow quotient $G_{n, k} / /\left(\mathbb{C}^{*}\right)^{n}$ is isomorphic to the Chow quotient $\left(\mathbb{C} P^{k-1}\right)^{n} / / P G L(k)$. In the case when $k=2$ and $n=5$, combining our results with the results of Kapranov [15] and Keel [18], we obtain here that the Chow quotient $\left(\mathbb{C} P^{1}\right)^{5} / / P G L(2, \mathbb{C})$ coincides with our universal space of parameters $\tilde{\mathcal{F}}$. Inspired by our results on description of $\tilde{\mathcal{F}}$ for $G_{5,2}$ presented in this paper, Klemyatin in [21] proved that the universal space of parameters for $G_{n, 2}$ coincides with the Chow quotient $\left(\mathbb{C} P^{1}\right)^{n} / / P G L(2, \mathbb{C})$. This result is worthy of attention, since it implies that the universal space of parameters $\tilde{\mathcal{F}}$ of the Grassmann manifolds $G_{n, 2}$ coincides with the well known moduli space $\overline{M_{0, n}}$ of stable $n$-points curves of genus zero which is a smooth compact algebraic variety, see [15].

Our approach gives a method to prove the following result:

Theorem 1.1. The orbit space $G_{n, 2} / T^{n}$ is a quotient space of the space $\mathcal{E}$ by an equivalence relation defined by the map $H$. 
The proof is technically complicated and to overcome difficulties we essentially use the fact that the symmetric group $S_{n}$ acts on the space $G_{n, 2} / T^{n}$ and that all our constructions are compatible with this action. In our previous paper [7] we proved this result for $n=4$ and reprove it here in Example 11.6. In this paper, we demonstrate the proof with all the details for $n=5$.

In [7] it was also proved that the space $G_{4,2} / T^{4}$ is homeomorphic to $\partial \Delta_{4,2} * \mathbb{C} P^{1}$.

The main consequences of the results of this paper are the following:

Theorem 1.2. There exists a continuous map $\left(G_{5,2} / T^{5}\right) /\left(G_{4,2} / T^{4}\right) \rightarrow \partial \Delta_{5,2} * \mathbb{C} P^{2}$ which induces an isomorphism in the integral homology groups and, thus, define a homotopy equivalence between these spaces.

Theorem 1.3. The orbit space $G_{5,2} / T^{5}$ is homotopy equivalent to the space obtained by attaching the disc $D^{8}$ to the space $\Sigma^{4} \mathbb{R} P^{2}$ by the generator of the group $\pi_{7}\left(\Sigma^{4} \mathbb{R} P^{2}\right)=\mathbb{Z}_{4}$.

Note that the following fact has been used for the proof of Theorem 1.3. Any of the coordinate embeddings $\mathbb{C}^{n-1} \rightarrow \mathbb{C}^{n}$ gives the $T^{n}$-equivariant embedding $G_{n-1, k} \rightarrow G_{n, k}$ such that the complement $G_{n, k} \backslash G_{n-1, k}$ can be identified with the space of the canonical vector bundle $\eta_{n-1, n-k}$ over the Grassmann manifold $G_{n-1, k-1}$. In this way we obtain the $T^{n}$-pair $\left(G_{n, k}, G_{n-1, k}\right)$ which defines $T^{n}$-action on the Thom space $T\left(\eta_{n-1, n-k}\right)$. Furthermore, the corresponding orbit space $T\left(\eta_{n-1, n-k}\right) / T^{n}$ is homeomorphic to the space obtained from $G_{n, k} / T^{n}$ by collapsing $G_{n-1, k} / T^{n-1}$ into the point. In particular, $\partial \Delta_{5,2} * \mathbb{C} P^{2} \cong T\left(\eta_{4,3}\right) / T^{5}$.

We show that there exists a $T^{n}$-equivariant smooth closed submanifold $S_{n, k}$ in $G_{n, k}$ of codimension 1 and $T^{n}$-equivariant projections $\pi_{0}: S_{n, k} \rightarrow G_{n-1, k}$ and $\pi_{1}: S_{n, k} \rightarrow G_{n-1, k-1}$ such that the following result holds.

Theorem 1.4. There exist projections $\hat{\pi}_{0}: S_{n, k} / T^{n} \rightarrow G_{n-1, k} / T^{n-1}$ and $\hat{\pi}_{1}: S_{n, k} / T^{n} \rightarrow$ $G_{n-1, k-1} / T^{n-1}$ such that

$$
G_{n, k} / T^{n}=\left(S_{n, k} / T^{n} \times[0,1]\right) \cup_{\hat{\pi}_{0}} G_{n-1, k} / T^{n-1} \cup_{\hat{\pi}_{1}} G_{n-1, k-1} / T^{n-1},
$$

where $[(s, 0)] \simeq \hat{\pi}_{0}[s]$ and $[(s, 1)] \simeq \hat{\pi}_{1}[s]$.

We want to emphasize that this paper is important for our work on developing the theory of $(2 l, q)$-manifolds, [4], [8]. These are $2 l$-dimensional manifolds with an effective action of the compact $q$-dimensional torus and an analogue of the moment map $M^{2 l} \rightarrow P^{q}$, where $P^{q}$ is a $q$ dimensional polytope. Our aim is to develop the methods of toric topology which enabled us to describe the action of the torus $T^{q}$ on $M^{2 l}$ in terms of the combinatorics of $P^{q}$ and the structure of the universal space of parameters $\tilde{\mathcal{F}}$ of the dimension $2(l-q)$.

An important invariant of $(2 l, q)$-manifolds is the complexity $d\left(M^{2 l}, q\right)=l-q$, which is equal to zero for quasitoric manifolds. This notion is used in symplectic geometry, where the Hamiltonian torus action of complexity 1 on a symplectic manifold is studied [16], [17], in algebraic geometry, where the complexity is defined as a codimension of the principal orbit for the action of algebraic torus [23], [26] and in equivariant topology [2], [3], [24].

The manifolds $G_{n, k}$ represent a key family of $(2 l, q)$-manifolds, where $l=k(n-k)$ and $q=$ $n-1$. The complexity for $G_{n, k}$ is $(k-1)(n-k-1)$ and, in this context, the spaces $G_{4,2}$ and $G_{5,2}$ are of special importance, since they provide nontrivial examples of the manifolds whose complexity is 1 and 2 , respectively.

In our theory of $(2 l, q)$-manifolds there are also other two important families. The first one is the family of the complex flag manifolds $F l(n)=U(n) / T^{n}$ with the canonical action of the torus $T^{n}$ and an analogue of the moment map $\mu: F l(n) \rightarrow P e^{n-1}$, where $P e^{n-1}$ is a permutahedron. 
For these manifolds $l=\left(\begin{array}{l}n \\ 2\end{array}\right), q=n-1$ and $d=\left(\begin{array}{c}n-1 \\ 2\end{array}\right)$. The second one consists of the manifolds $\mathbb{C} P^{N-1}, N=\left(\begin{array}{l}n \\ 2\end{array}\right)$ with the action of the torus $T^{n}$ which is given as a composition of the second exterior power representation $T^{n} \rightarrow T^{N}$ and the standard action of $T^{N}$ on $\mathbb{C} P^{N-1}$, and an analogue of the moment map $\mu: \mathbb{C} P^{N-1} \rightarrow \Delta_{n, 2}$. For these manifolds $q=N-1, l=n-1$ and $d=N-n$. We proved that for $(6,2)$-manifold $F l(3)$ with $d=1$ there is a homeomorphism $F l(3) / T^{3} \cong S^{4}$ and for $(10,3)$-manifold $\mathbb{C} P^{5}$ with $d=2$ there is a homeomorphism $\mathbb{C} P^{5} / T^{4} \cong \partial \Delta_{4,2} * \mathbb{C} P^{2}$, [7].

The canonical action of the algebraic torus $\left(\mathbb{C}^{*}\right)^{n}$ on the manifold $G_{n, k}$ enables us to assign to each point from $G_{n, k}$ a toric variety, that is the closure of $\left(\mathbb{C}^{*}\right)^{n}$-orbit of this point. It naturally arises the problem: classify, up to an algebraic equivalence, all such toric varieties. In [7] this problem was solved for $G_{4,2}$, while in the current paper it is solved for the manifold $G_{5,2}$. In [22] in the frame of this problem, all such smooth toric subvarieties in the Grassmann manifod $G_{n, k}$ are described for any $n$ and $k$. The next natural step in this direction would be the question: classify all such toric subvarieties in $G_{n, k}$ whose singularities are only the fixed points. An answer to this question for the case $G_{4,2}$ and $G_{5,2}$ is given in [7] and in current paper, respectively.

Acknowledgment. We are grateful to Anton Ayzenberg, Alexander Gaifullin, Nikita Klemyatin, Yael Karshon, Mikiya Masuda, Taras Panov and Hendrik Süss for useful and fruitful discussions on the results of this paper.

\section{The CANONICAL ACTION of $T^{n}$ ON $G_{n, k}$}

The complex Grassmann manifolds $G_{n, k}=G_{n, k}(\mathbb{C})$ consist of all $k$-dimensional complex subspaces in $\mathbb{C}^{n}$. The manifolds $G_{n, k}$ and $G_{n, n-k}$ are diffeomorphic. For the standard scalar product in $\mathbb{C}^{n}$ there is the canonical diffeomorphism $c_{n k}: G_{n, k} \rightarrow G_{n, n-k}$ which sends any $k$-dimensional subspace of $\mathbb{C}^{n}$ sends to its orthogonal complement. The coordinate-wise action of the compact torus $T^{n}$ on $\mathbb{C}^{n}$ induces the canonical action of $T^{n}$ on $G_{n, k}$. The canonical diffeomorphism $c_{n k}$ is equivariant for this action. One of the well known and important problems is the description of the combinatorial structure and algebraic topology of the orbit space $G_{n, k} / T^{n} \cong G_{n, n-k} / T^{n}$.

Example 2.1. The manifold $G_{n, 1}=\mathbb{C} P^{n-1}$ is a toric manifold and its orbit space $G_{n, 1} / T^{n}$ is homeomorphic to the standard simplex $\Delta^{n-1}$ in $\mathbb{R}^{n}$.

2.1. Plücker coordinates. Let us fix a basis in $\mathbb{C}^{n}$ and fix a basis in $L \in G_{n, k}$. In these base, $L$ can be be represented by the $(n \times k)$ - matrix $A_{L}$. Denote by $P^{I}\left(A_{L}\right)$ a $(k \times k)$ - minor of $A_{L}$, which is determined by the rows indexed by the elements of $I$, where $I \subset\{1, \ldots, n\}$ such that $|I|=k$. The complex numbers $\left(P^{I}\left(A_{L}\right)\right)$, where $I$ runs through all subsets of $\{1, \ldots n\}$ such that $|I|=k$, are known as the Plücker coordinates of the subspace $L \subset \mathbb{C}^{n}$. The Plücker coordinates $\left(P^{I}(L)\right)$ are defined uniquely, up to common scalar, and they give the Plücker embedding $G_{n, k} \rightarrow \mathbb{C} P^{N-1}$, $N=\left(\begin{array}{l}n \\ k\end{array}\right)$ which is defined by

$$
L \rightarrow P(L)=\left(P^{I}\left(A_{L}\right)\right), \quad I \subset\{1, \ldots n\},|I|=k
$$

The Plücker embedding provides $G_{n, k}$ with the structure of a complex $k(n-k)$-dimensional algebraic projective manifold.

Example 2.2. The image of the Plücker embedding of $G_{4,2}$ in $\mathbb{C} P^{5}$ is the hypersurface

$$
z_{12} z_{34}+z_{14} z_{23}=z_{13} z_{24} .
$$


Example 2.3. The image of the Plücker embedding of $G_{5,2}$ in $\mathbb{C} P^{9}$ is the intersection of the five hypersurfaces:

$$
\begin{gathered}
z_{12} z_{34}+z_{14} z_{23}=z_{13} z_{24}, \quad z_{12} z_{35}+z_{15} z_{23}=z_{13} z_{25}, \\
z_{12} z_{45}+z_{15} z_{24}=z_{14} z_{25}, \quad z_{13} z_{45}+z_{15} z_{34}=z_{14} z_{35}, \quad z_{23} z_{45}+z_{25} z_{34}=z_{24} z_{35} .
\end{gathered}
$$

Example 2.4. The image of the Plücker embedding of $G_{n, 2}, n \geq 4$ in $\mathbb{C} P^{N-1}, N=\left(\begin{array}{l}n \\ 2\end{array}\right)$ is the intersection of the $\left(\begin{array}{l}n \\ 4\end{array}\right)$ quadratic hypersurfaces:

$$
z_{i j} z_{k l}+z_{j k} z_{i l}=z_{i k} z_{j l}, \quad 1 \leq i<j<k<l \leq n .
$$

Remark 2.5. Note that the normal bundle for $G_{n, 2}$ in $\mathbb{C} P^{N-1}$ is a complex vector bundle of the dimension $\left(\begin{array}{c}n-2 \\ 2\end{array}\right)$. Thus, for $n>4$ we have the algebraic manifold $G_{n, 2} \subset \mathbb{C} P^{N-1}$ without singularities, which is not a complete intersection.

Let $\rho_{n, k}: T^{n} \rightarrow \mathbb{T}^{N}, N=\left(\begin{array}{l}n \\ k\end{array}\right)$ be a representation given by the $k$-th exterior power

$$
\left(t_{1}, \ldots, t_{n}\right) \rightarrow\left(t_{1} \cdots t_{k}, \ldots, t_{n-k+1} \cdots t_{n}\right) .
$$

Consider the action of $T^{n}$ on $\mathbb{C} P^{N-1}, N=\left(\begin{array}{l}n \\ k\end{array}\right)$, which is given as a composition of the standard action of $T^{N}$ on $\mathbb{C} P^{N-1}$ and the representation $\rho_{n, k}$. It directly follows:

Lemma 2.6. The Plücker embedding

$$
T^{n} \curvearrowright G_{n, k} \stackrel{\rho_{n, k}}{\rightarrow} \mathbb{C} P^{N-1} \curvearrowleft T^{n}
$$

is equivariant for the canonical action of $T^{n}$ on $G_{n, k}$ and the $k$-th exterior power action of $T^{n}$ on $\mathbb{C} P^{N-1}$.

The standard moment map $\mu: \mathbb{C} P^{N-1} \rightarrow \Delta^{N-1} \subset \mathbb{R}^{N}$ for $T^{N}$-action on $\mathbb{C} P^{N}$ is given by

$$
\mu(\mathbf{z})=\frac{1}{|\mathbf{z}|^{2}}\left(\left|z_{1}\right|^{2}, \ldots,\left|z_{N}\right|^{2}\right)
$$

where $\mathbf{z}=\left(z_{1}: \ldots: z_{N}\right),|\mathbf{z}|^{2}=\sum_{i=1}^{N}\left|z_{i}\right|^{2}$ and $\Delta^{N-1}$ is the standard simplex. In this way it is defined the map

$$
\mu \circ \rho_{n, k}: G_{n, k} \rightarrow \Delta^{N-1}
$$

2.2. Moment map. Let $\mathbb{R}^{n}$ be $n$-dimensional real vector space with a fixed basis. The weight vectors of the representation $\rho_{n, k}$ are:

$$
\Lambda_{I} \in \mathbb{Z}^{n} \subset \mathbb{R}^{n}, \quad\left(\Lambda_{I}\right)_{j}=1 \text { for } j \in I, \quad\left(\Lambda_{I}\right)_{j}=0 \text { for } j \notin I,
$$

where $I \subset\{1, \ldots, n\},|I|=k$. In other words, $\Lambda_{I}$ has 1 at $k$ places and it has 0 at the other $(n-k)$ places.

The moment map $\mu: G_{n, k} \rightarrow \mathbb{R}^{n}$, see [20], [12] is defined by

$$
\mu(L)=\frac{1}{|P(L)|^{2}} \sum\left|P^{I}\left(A_{L}\right)\right|^{2} \Lambda_{I}, \quad|P(L)|^{2}=\sum\left|P^{I}\left(A_{L}\right)\right|^{2},
$$

where $\Lambda_{I} \in R^{n}, \quad\left(\Lambda_{I}\right)_{j}=1$ for $j \in I, \quad\left(\Lambda_{I}\right)_{j}=0$ for $j \notin I$ and the sum goes over all subsets $I \subset\{1, \ldots, n\},|I|=k$. 
2.3. Hypersimplex. The image of $\mu$ is the polytope which is obtained as the convex hull of the vectors $\Lambda_{I}$. This polytope is known as the hypersimplex and it is denoted by $\Delta_{n, k}$. More precisely,

$$
\Delta_{n, k}=I^{n} \cap\left\{\left(x_{1}, \ldots, x_{n}\right) \in \mathbb{R}^{n}, \sum_{i=1}^{n} x_{i}=k\right\} .
$$

It follows that $\Delta_{n, k}$ belongs to the hyperplane $x_{1}+\cdots+x_{n}=k$ in $\mathbb{R}^{n}$ which implies that $\Delta_{n, k}$ is a $(n-1)$-dimensional polytope.

Example 2.7. $\Delta_{n, 1}$ is the standard simplex $\Delta^{n-1}$, while $\Delta_{4,2}$ is the octahedron.

The hypersimplex $\Delta_{n, k}$ has $\left(\begin{array}{l}n \\ k\end{array}\right)$ vertices, $2 n$ facets and $k(n-k)$ edges meet at every vertex. The set of facets for $\Delta_{n, k}$ can be expressed as a union of $n$ copies of $\Delta_{n-1, k}$ and $n$ copies of $\Delta_{n-1, k-1}$, [27]. We shortly describe how these facets arise from the Grassmannian $G_{n, k}$.

The embeddings $i_{q}: \mathbb{C}^{n-1} \rightarrow \mathbb{C}^{n}, i_{q}\left(z_{1}, \ldots, z_{n-1}\right)=\left(z_{1}, \ldots z_{q-1}, 0, z_{q}, \ldots, z_{n-1}\right), 1 \leq q \leq$ $n-1$ are equivariant for the coordinate-wise torus action and they induce the embeddings $\tilde{i_{g}}$ : $G_{n-1, k} \rightarrow G_{n, k}$ and $\hat{i_{q}}: G_{n-1, k-1} \rightarrow G_{n, k}$ for any $n \in \mathbb{N}$, whose images we denote by $G_{n-1, k}(q)$ and $G_{n-1, k-1}(q)$, respectively. These embeddings are equivariant for the corresponding embedding of the standard tori which implies that they are compatible with the moment map. Therefore they induce the embeddings

$$
\Delta_{n, k-1}(q) \rightarrow \Delta_{n+1, k} \text { and } \Delta_{n, k}(q) \rightarrow \Delta_{n+1, k}, \quad 1 \leqslant q \leqslant n+1 .
$$

It is straightforward to see that $\Delta_{n-1, k}(q) \subset I_{x_{q}=0}^{n}, \Delta_{n-1, k-1}(q) \subset I_{x_{q}=1}^{n}$, and $\Delta_{n-1, k}(q), \Delta_{n-1, k-1}(q)$ belong to the boundary of $\Delta_{n, k}$ for all $1 \leq q \leq n$.

Remark 2.8. We note that the set of vertices for $\Delta_{n, k}$ decomposes into two subsets according to the formula $\left(\begin{array}{l}n \\ k\end{array}\right)=\left(\begin{array}{c}n-1 \\ k\end{array}\right)+\left(\begin{array}{l}n-1 \\ k-1\end{array}\right)$. There are $n$ such decompositions given by the pairs $\left(\Delta_{n-1, k}(q), \Delta_{n-1, k-1}(q)\right)$, $1 \leqslant q \leqslant n$. In this way we obtain the combinatorial structure on the sphere $S^{n-2}$. The symmetric group $S_{n}$ is a symmetry group for this structure. More precisely this combinatorial structure is given by the orbit of the pair $\left(\Delta_{n-1, k}(1), \Delta_{n-1, k-1}(1)\right)$ by an action of the group $S_{n}$.

2.4. Admissible polytopes and strata. Using Plücker coordinates it can be defined a smooth atlas on $G_{n, k}$. The charts are given by $M_{I}=\left\{L \in G_{n, k}: P^{I}(L) \neq 0\right\}, I \subset\{1, \ldots, n\},|I|=k$ and the homeomorphisms $u_{I}: M_{I} \rightarrow \mathbb{C}^{k(n-k))}$ are constructed as follows. Any $L \in M_{I}$ can be uniquely represented by the $(n \times k)$-matrix $A_{L}$ whose submatrix, determined by the rows indexed by $I$ is an identity matrix. In this way, the matrix $A_{L}$ has $k(n-k)$-variables $a_{i j}(L)$ and the homeomorphism $u_{I}: M_{I} \rightarrow \mathbb{C}^{k(n-k)}$ is given by $u_{I}(L)=\left(a_{i j}(L)\right)$, where $i \notin I$. It can be easily seen that any chart $M_{I}$ contains exactly one fixed point given by the element $L$ such that $u_{i}(L)=0 \in \mathbb{C}^{k(n-k)}$. The number of charts is $m=\left(\begin{array}{l}n \\ k\end{array}\right)$ and it coincides with the number of fixed points for the canonical $T^{n}$-action on $G_{n, k}$. The charts $M_{I}$ are open, $T^{n}$ invariant and dense sets in $G_{n, k}$. It follows that the sets $Y_{I}=G_{n, k} \backslash M_{I}$ are closed and $T^{n}$-invariant.

Let us enumerate the charts as $\left(M_{I_{1}}, u_{I_{1}}\right), \ldots,\left(M_{I_{m}}, u_{I_{m}}\right)$. Define the spaces $W_{\sigma}$, where $\sigma=$ $\left\{I_{1}, \ldots, I_{l}\right\}$ and $I_{i} \subset\{1, \ldots, n\}$ such that $\left|I_{i}\right|=k, 1 \leq i \leq l$ and $1 \leq l \leq m$, by

$$
W_{\sigma}=M_{I_{1}} \cap \cdots M_{I_{l}} \cap Y_{I_{l+1}} \cap \cdots Y_{I_{m}},
$$

where $\left\{I_{l+1}, \ldots, I_{m}\right\}=\left\{I_{1}, \ldots, I_{m}\right\} \backslash\left\{I_{1}, \ldots, I_{l}\right\}$. More precisely,

$$
W_{\sigma}=\left\{L \in G_{n, k}: P^{I}(L) \neq 0 \text { for } I \in \sigma \text { and } P^{I}(L)=0 \text { for } I \notin \sigma\right\} \text {, }
$$


Definition 2.9. A non-empty space $W_{\sigma}$ is said to be the stratum . The index set $\sigma$ of a stratum $W_{\sigma}$ is said to be an admissible set.

Definition 2.10. The stratum $W_{\sigma}=M_{I_{1}} \cap \cdots \cap M_{I_{m}}$, where $\sigma=\left\{I_{1}, \ldots, I_{m}\right\}$ we call the main stratum and denote by $W$.

We denote by $\mathcal{A}$ the set of all admissible sets.

Lemma 2.11. For $\sigma=\{I\}, W_{\sigma}$ is a fixed point.

Proof. In this case, any element $L \in W_{\sigma}$ can be represented by the matrix $A$ such that $A_{I}=I_{d}$ and any $k \times k$-minor of $A$ different from that one given by $A_{I}$ is zero. This implies that all elements of $A$ apart from $A_{I}$ are zero, which implies that $W_{\sigma}$ consists of one element that is a fixed point.

Lemma 2.12. The strata $W_{\sigma}$ for $G_{n, k}$ are disjoint subspaces which are $T^{n}$-invariant and give the equivariant subdivision $G_{n, k}=\cup_{\sigma} W_{\sigma}$.

From the definition of the moment map it follows that $\mu\left(W_{\sigma}\right)=\stackrel{\circ}{P_{\sigma}}$, where $P_{\sigma}$ is the convex hull of the points $\Lambda_{I_{1}}, \ldots, \Lambda_{I_{l}}$ and $\sigma=\left\{I_{1}, \ldots, I_{l}\right\}$. In particular for $l=1$ it holds $\mu\left(W_{\sigma}\right)=\Lambda_{I}$.

Definition 2.13. A polytope $P_{\sigma}$ in $\Delta_{n, k}$, whose interior is the image of the stratum $W_{\sigma}$ by the moment map that is $\mu\left(W_{\sigma}\right)=\stackrel{\circ}{P}_{\sigma}$ is called the admissible polytope.

2.5. $\left(\mathbb{C}^{*}\right)^{n}$-action on $G_{n, k}$. The canonical action of $T^{n}$ on $G_{n, k}$ extends to a canonical action of the algebraic torus $\left(\mathbb{C}^{*}\right)^{n}$ on $G_{n, k}$. The strata we $W_{\sigma}$ are invariant for the action of the algebraic torus $\left(\mathbb{C}^{*}\right)^{n}$ as well.

Definition 2.14. The space $F_{\sigma}=W_{\sigma} /\left(\mathbb{C}^{*}\right)^{n}$ is called the space of parameters of the stratum $W_{\sigma}$.

The space of parameters of the main stratum $W$ is denoted by $F=F_{n, k}$. We will omit the indices $n, k$ when it is clear from the context that the word is about the main stratum.

The moment map $\mu: G_{n, k} \rightarrow \Delta_{n, k}$ relates the $\left(\mathbb{C}^{*}\right)^{n}$-orbits on $G_{n, k}$ and some polytopes in $\Delta_{n, k}$. More precisely, the classical convexity theorem of [1], [10] states:

Theorem 2.15. Let $\mathcal{O}_{\mathbb{C}}(L)$ be an orbit of an element $L \in G_{n, k}$ under the canonical action of $\left(\mathbb{C}^{*}\right)^{n}$. Then $\mu\left(\overline{\mathcal{O}_{\mathbb{C}}(L)}\right)$ is a convex polytope in $\mathbb{R}^{n}$ whose vertex set is given by $\left\{\Lambda_{I} \mid P^{I}(L) \neq 0\right\}$. The mapping $\mu$ gives a bijection between p-dimensional orbits of the group $\left(\mathbb{C}^{*}\right)^{n}$ in $\overline{\mathcal{O}_{\mathbb{C}}(L)}$ and p-dimensional open faces of the polytope $\mu\left(\overline{\mathcal{O}_{\mathbb{C}}(L)}\right)$.

The closure $\overline{\mathcal{O}_{\mathbb{C}}(L)}$ is a toric variety for any $L \in G_{n, k}$. This toric variety is a smooth manifold depending if the corresponding admissible polytope is a simple polytope or not. A natural question is to describe the singularities of these toric varieties. It is done in [7] for the case of $G_{4,2}$, while in this paper we do it for $G_{5,2}$ by Theorem 4.9 .

Remark 2.16. A notion of the stratum in $G_{n, k}$ is defined in [12], [13] by different methods in three equivalent ways. By the one of these definitions two points $L_{1}, L_{2} \in G_{n, k}$ are said to belong to the same stratum in $G_{n, k}$ if

$$
\mu\left(\overline{\mathcal{O}_{\mathbb{C}}\left(L_{1}\right)}\right)=\mu\left(\overline{\mathcal{O}_{\mathbb{C}}\left(L_{2}\right)}\right) .
$$

In other words, by [12] the stratum in $G_{n, k}$ consists of all $\left(\mathbb{C}^{*}\right)^{n}$-orbits which are mapped by the moment map to the same polytope in $\Delta_{n, k}$. It is straightforward to verify that our definition of strata is equivalent to this definition. 
Remark 2.17. We want to note that our approach to the notion of strata given by (3) is more general since it does not use the existence of $\left(\mathbb{C}^{*}\right)^{n}$-action on $G_{n, k}$, which extends $T^{n}$-action. This approach is fundamental for developing the theory of $(2 l, q)$-manifolds.

2.6. The space of parameters of the main stratum. Let us consider the main stratum $W \subset G_{n, k}$ and a point $L \in W$. The subspace $L$ can be described by a matrix and this matrix can be considered as $n$ - tuple of $k$-dimensional vectors. Any $k$ vectors from this $n$-tuple define the Plücker coordinate, which is non-zero, and, therefore, these vectors are linearly independent. In this way, we obtain the map $W \rightarrow\left(\mathbb{C}^{k} \backslash \mathbf{0}\right)^{n} / G L(k, \mathbb{C})$. This map is invariant for $\left(\mathbb{C}^{*}\right)^{n}$-action and it induces an embedding

$$
F=W /\left(\mathbb{C}^{*}\right)^{n} \rightarrow\left(\mathbb{C} P^{k-1}\right)^{n} / P G L(k, \mathbb{C}) .
$$

Note that this map plays an important role in [15].

Let $k=2$ and $\mathcal{W}_{n, 2}=\left\{\left(\left(z_{1}: z_{1}^{\prime}\right), \ldots,\left(z_{n}: z_{n}^{\prime}\right)\right) \in\left(\mathbb{C} P^{1}\right)^{n} \mid\left(z_{i}: z_{i}^{\prime}\right) \neq\left(z_{j}: z_{j}^{\prime}\right)\right\}$.

Lemma 2.18. The projective group $P G L(2, \mathbb{C})$ acts freely on $\mathcal{W}_{n, 2}$ and $F \cong \mathcal{W}_{n, 2} / P G L(2, \mathbb{C})$.

Proof. The matrices which represent the elements from the main stratum in $G_{n, 2}$ are characterized by the condition that all their $(2 \times 2)$-minors are non-zero. This condition is equivalent to the condition that the image of the map $W \rightarrow\left(\mathbb{C}^{2} \backslash \mathbf{0}\right)^{n} / G L(2, \mathbb{C})$ consists of $n$-tuples of pairs, which are pairwise non-collinear. It implies that $F \cong \mathcal{W}_{n, 2} / P G L(2, \mathbb{C})$.

It is a classical result that $\mathcal{W}_{3,2} / P G L(2, \mathbb{C})$ is a point. For arbitrary $n$ we prove:

Proposition 2.19. The space of parameters $F$ is homeomorphic to

$$
\left(\mathbb{C} P^{1}\right)^{n-3} \backslash G_{n}, \quad G_{n}=\left(\cup_{j=1}^{n}\left(A \times_{j}\left(\mathbb{C} P^{1}\right)^{n-1}\right)\right) \cup\left(\cup_{1 \leq i<j \leq n} \Delta_{i, j}\left(\mathbb{C} P^{1}\right)\right),
$$

where $A=\{(1: 0),(0: 1),(1: 1)\}, A \times{ }_{j}\left(\mathbb{C} P^{1}\right)^{n-1}=\mathbb{C} P^{1} \times \cdots \times \mathbb{C} P^{1} \times \stackrel{j}{A} \times \mathbb{C} P^{1} \times \cdots \times \mathbb{C} P^{1}$ and $\Delta_{i, j}\left(\mathbb{C} P^{1}\right) \subset\left(\mathbb{C} P^{1}\right)^{n-3}$ is given by the diagonal on $i$-th and $j$-th factor.

Proof. It follows from Lemma 2.18 that $F \cong \mathcal{W}_{n, 2} / P G L(2, \mathbb{C})$. Let $\left(z_{1}, \ldots, z_{n}\right) \in \mathcal{W}_{n, 2}$. Since any three different points $z_{1}, z_{2}, z_{3}$ in $\mathbb{C} P^{1}$ produce a projective basis for $\mathbb{C} P^{1}$, there is a unique homography defined by $z_{1} \rightarrow(1: 0), z_{2} \rightarrow(0: 1)$ and $z_{3} \rightarrow(1: 1)$. Then the mapping $\mathcal{W}_{n, 2} \rightarrow\left(\mathbb{C} P^{1}\right)^{n-3}$ is given by the cross-ratio $z_{i} \rightarrow\left[z_{1}, z_{2}, z_{3}, z_{i}\right]$ of the points $z_{1}, z_{2}, z_{3}, z_{i}$. It implies that $\mathcal{W}_{n, 2} / P G L(2, \mathbb{C}) \cong\left\{\left(z_{4}, \ldots, z_{n}\right) \in\left(\mathbb{C} P^{1}\right)^{n-3}, z_{i} \notin A, z_{i} \neq z_{j}\right\}$, which proves the statement.

Corollary 2.20. $F_{4,2}$ is homeomorphic to $\mathbb{C} P^{1} \backslash A$ and $F_{5,2}$ is homeomorphic to $\left(\mathbb{C} P^{1} \times \mathbb{C} P^{1}\right) \backslash G_{5}$, where $G_{5}=\left(A \times \mathbb{C} P^{1}\right) \cup\left(\mathbb{C} P^{1} \times A\right) \cup \Delta_{1,2}\left(\mathbb{C} P^{1}\right)$.

Remark 2.21. The space $F_{5,2}$ is a two-dimensional open algebraic manifold. In order to describe the orbit space $G_{5,2} / T^{5}$ it is necessary to find a compactification of the space $F_{5,2}$ that corresponds to the compactification of the orbit space of the main stratum $W / T^{5}$ in $G_{5,2} / T^{5}$.

Proposition 2.22. The non-point space of parameters $F_{\sigma}$ of a stratum $W_{\sigma}$ in $G_{n, 2}$, which is different from the main stratum, can be embedded in $\left(\mathbb{C} P^{1}\right)^{l}$ for $1 \leq l \leq n-4$.

Proof. Since $W_{\sigma}$ is not the main stratum its points have at least one common zero Plücker coordinate. It implies that at least two rows in the matrix representing a point of this stratum are collinear. Note that the condition that $F_{\sigma}$ is not a point implies that the matrices which represent $W_{\sigma}$ must have at least four rows such that any two of them are non-collinear. Therefore, $W_{\sigma} /\left(\mathbb{C}^{*}\right)^{n}$ can be 
embedded in $\left(\mathbb{C} P^{1}\right)^{s} / P G L(2, \mathbb{C}), 4 \leq s \leq n-1$, which implies that $F_{\sigma}$ can be embedded in $\left(\mathbb{C} P^{1}\right)^{s-3}$.

2.7. Action of the symmetric group $S_{n}$. The symmetric group $S_{n}$ acts on $\mathbb{C}^{n}$ by permuting coordinates. This action induces the action of $S_{n}$ on $G_{n, k}$.

The $S_{n}$-action on $G_{n, k}$ can be interpreted as a $S_{n}$-action on the corresponding $(n \times k)$ - matrices $A_{L}$, which is given by the permutation of the rows. It implies that $L_{1}, L_{2} \in G_{n, k}$ has the same nonzero Plücker coordinates if and only if $s\left(L_{1}\right), s\left(L_{2}\right)$ has the same non-zero Plücker coordinates for any $s \in S_{n}$. In other words $L_{1}, L_{2}$ belong to the same stratum if and only if $s\left(L_{1}\right), s\left(L_{2}\right)$ belong to the same stratum, for any $s \in S_{5}$. Therefore the group $S_{n}$ acts on the set of all strata for $G_{n, k}$. Since this action is equivariant relative to the action of the torus $\left(\mathbb{C}^{*}\right)^{n}$, we deduce:

Lemma 2.23. The canonical action of $S_{n}$ on $G_{n, k}$ induces an $S_{n^{-}}$action on the set of admissible polytopes and the set of spaces of parameters of the strata by $s\left(P_{\sigma}\right)=\mu\left(s\left(W_{\sigma}\right)\right)$ and $s\left(F_{\sigma}\right)=$ $s\left(W_{\sigma}\right) /\left(\mathbb{C}^{*}\right)^{n}$.

Lemma 2.24. The strata $W_{\sigma}$ and $s\left(W_{\sigma}\right)$ belongs to the same number of charts for any stratum $W_{\sigma}$ and any $s \in S_{n}$. Consequently, the admissible polytopes $P_{\sigma}$ and $s\left(P_{\sigma}\right)$ have the same number of vertices.

Proof. The statement directly follows from the fact that the strata $W_{\sigma}$ and $s\left(W_{\sigma}\right)$ have the same number of non-zero Plücker coordinates and that the vertices of an admissible polytope are determined by the non-zero Plücker coordinates of the corresponding stratum.

We elaborate this action in more detail. Let $S[n]$ be the power set of $\{1, \ldots, n\}$. Then $S_{n}$ acts on $S[n]$ and $|\sigma|=|s(\sigma)|$ for $s \in S_{n}, \sigma \in S[n]$. It implies that $S_{n}$ acts on the set $W(p)=\left\{W_{\sigma},|\sigma|=\right.$ $p\}$ for any fixed $p, 1 \leq p \leq N$. Put $m_{p}=|W(p)|$ and let $S_{\sigma}(p)$ be a subgroup of $S_{n}$, which is a stabilizer for $W_{\sigma}$, where $W_{\sigma} \in W(p)$.

If the orbit $S_{n}\left(W_{\sigma}\right)$ coincides with $W(p)$, then $m_{p}=\frac{n !}{\left|S_{\sigma}(p)\right|}$ and there is one $S_{n}$-generator $W_{\sigma_{1_{p}}}$ for $W(p)$. Otherwise, $S_{n}$ acts on $W(p) \backslash S_{n}\left(W_{\sigma_{1}}\right)$, we repeat the argument and obtain the next generator $W_{\sigma_{2_{p}}}$. In this way we obtain the set of generators $W_{\sigma_{1_{p}}}, \ldots, W_{\sigma_{q_{p}}}$.

Definition 2.25. The strata $W_{\sigma_{i_{p}}}, 1 \leq i \leq q$ are said to be the fundamental strata for a given $p$, where $q=q_{p}$.

From the description of the fundamental strata we obtain:

$$
m_{p}=\sum_{i=1}^{q} \frac{n !}{\left|S_{\sigma_{i_{p}}}(p)\right|},
$$

Corollary 2.26. There exist integer functions $m_{p}, q_{p}:[1, N] \rightarrow \mathbb{Z}, N=\left(\begin{array}{l}n \\ k\end{array}\right)$ which are invariants for $T^{n}$-action on $G_{n, k}$.

Definition 2.27. Admissible polytopes which correspond to the fundamental strata are called the fundamental polytopes.

Thus, any admissible polytope can be obtained by the action of the group $S_{n}$ on some of the fundamental polytopes. 
2.8. The $T^{n}$-action on the Thom spaces. We show that the canonical torus action on the complex Grassmann manifolds induces a torus action on the corresponding Thom spaces. Denote by $\xi_{n, k}$ and $\eta_{n, n-k}$ the canonical complex vector bundles over $G_{n, k}$, where $\operatorname{dim}_{\mathbb{C}} \xi_{n, k}=k, \operatorname{dim}_{\mathbb{C}} \eta_{n, n-k}=$ $n-k$. Moreover, it holds $\xi_{n, k} \oplus \eta_{n, n-k}=n(1)$.

Put $E_{1, q}=G_{n, k} \backslash G_{n-1, k-1}(q)$ and $E_{2, q}=G_{n, k} \backslash G_{n-1, k}(q)$. An $T^{n}$-equivariant projection $\pi_{1, q}$ : $E_{1, q} \rightarrow G_{n-1, k}$ is defined using the following observation. If $L \in E_{1, q}$ then $L$ does not contain the coordinate vector $e_{q}$ which implies that the retraction $r_{q}: \mathbb{C}^{n} \rightarrow \mathbb{C}_{z_{q}=0}^{n-1}$ maps $L$ to a $k$-dimensional subspace in $\mathbb{C}^{n-1}$. Thus, $\pi_{1, q}(L) \in G_{n, k}$ is defined by $r_{q}(L)$.

We define a $T^{n}$-equivariant projection $\pi_{2, q}: E_{2, q} \rightarrow G_{n-1, k-1}$ as the following composition:

$$
E_{2, q} \stackrel{c_{n, k}}{\longrightarrow} G_{n, n-k} \backslash G_{n-1, n-k-1} \stackrel{\pi_{1, q}}{\longrightarrow} G_{n-1, n-k} \stackrel{c_{n-1, n-k}}{\longrightarrow} G_{n-1, k-1} .
$$

We obtain the following, ( cf [14]):

Proposition 2.28. The equivariant projection $\pi_{1, q}: E_{1, q} \rightarrow G_{n-1, k}$ can be identified with the $T^{n}$ vector bundle $\xi_{n-1, k} \rightarrow G_{n-1, k}$, while the equivariant projection $\pi_{2, q}: E_{2, q} \rightarrow G_{n-1, k-1}$ can be identified with the $T^{n}$-vector bundle $\eta_{n-1, n-k} \rightarrow G_{n-1, k-1}$.

Proof. The homeomorphism $\xi_{n-1, k} \rightarrow E_{1, q}$ is defined by

$$
(x \in L, L) \rightarrow A_{q}(x, L)=\left(\begin{array}{c}
A_{x} \\
A_{L}
\end{array}\right)
$$

where $A_{L}$ is an $((n-1) \times k)$-matrix of the subspace $L$ in a fixed basis and $A_{x}$ is an $k$-vector which represents the vector $x$ in this basis. The notation $A_{q}(x, L)$ means that the vector $A_{x}$ is inserted as an $q$-th row of the matrix consisting of the matrices $A_{L}$ and $A_{x}$. Then the subspace $L \subset \mathbb{C}^{n}$ determined by the matrix $A_{q}(x, L)$ does not contain the coordinate vector $e_{q}$.

Corollary 2.29. For any $q, 1 \leqslant q \leqslant n$ there are two $T^{n}$-pairs $\left(G_{n, k}, G_{n-1, k-1}(q)\right)$ and $\left(G_{n, k}, G_{n-1, k}(q)\right)$ which define $T^{n}$-spaces $X_{n, k}(q)=T\left(\xi_{n-1, k}\right)$ and $Y_{n, k}(q)=T\left(\eta_{n-1, n-k}\right)$ that is the Thom spaces for $\xi_{n-1, k}$ and $\eta_{n-1, n-k}$.

Proof. Since $E_{1, q}=G_{n, k} \backslash G_{n-1, k-1}(q)$ is homeomorphic to $\xi_{n-1, k}$ it follows that $G_{n, k} / G_{n-1, k-1}(q)$ is homeomorphic to the Thom space $T\left(\xi_{n-1, k}\right)$. An action of $T^{n}$ on $T\left(\xi_{n-1, k}\right)$ is given by

$$
T^{n} \cdot(x, L)=T^{n} \cdot A_{q}(x, L)=A_{q}\left(T^{1} x, T^{n-1} L\right)=\left(\begin{array}{c}
T^{1} \cdot A_{x} \\
T^{n-1} \cdot A_{L}
\end{array}\right)
$$

where $T^{1}=T^{1}(q)$ is a subgroup of $T^{n}$ given by $z \rightarrow\left(z, \ldots, z, 1_{q}, z, \ldots, z\right)$ and $T^{n-1}=T^{n} / T^{1}$.

Note that a continuous action of a group $G$ on a topological space $X$ induces the $G$-action on the quotient space $X / Y$ for any $G$-invariant subspace $Y \subset X$. Moreover, $(X / Y) / G \cong$ $(X / G) /(Y / G)$. Therefore, the previous Corollary implies:

Corollary 2.30. The orbit spaces of the Thom spaces $X_{n, k}(q)=T\left(\xi_{n-1, k}\right)$ and $Y_{n, k}(q)=T\left(\eta_{n-1, n-k}\right)$ by the $T^{n}$-action are homeomorhic to the quotient spaces $\left(G_{n, k} / T^{n}\right) /\left(G_{n-1, k-1} / T^{n-1}\right)$ and $\left(G_{n, k} / T^{n}\right) /\left(G_{n-1, k} / T\right.$ respectively.

Since $G_{n-1,1}=\mathbb{C} P^{n-2}$ and $\mathbb{C} P^{n-2} / T^{n-2} \cong \Delta_{n-2}$, one has:

Corollary 2.31. The orbit space $X_{n, 2}(q) / T^{n}$ is homotopy equivalent to $G_{n, 2} / T^{n}$. 
In particular, since [7] gives that $G_{4,2} / T^{4} \cong S^{5}$, one has

Corollary 2.32. The orbit spaces $X_{4,2}(q) / T^{4}$ and $Y_{4,2}(q) / T^{4}$ are homotopy equivalent to $S^{5}$.

\section{ORBIT SPACES OF THE STRATA}

It is known that $G_{n, k} /\left(\mathbb{C}^{*}\right)^{n}$ is a non-Hausdorff topological space [11] for the topology induced from $G_{n, k}$. We prove that the spaces of parameters $F_{\sigma}$ as well as the orbit spaces $W_{\sigma} / T^{n}$ are much better behaved.

The charts $M_{I}$ are invariant under the action of $\left(\mathbb{C}^{*}\right)^{n}$ and this action via homeomorphism $u_{I}$ : $M_{I} \rightarrow \mathbb{C}^{k(n-k)}$ induces an action of $\left(\mathbb{C}^{*}\right)^{n}$ on $\mathbb{C}^{k(n-k)}$. For $I=\{1, \ldots, k\}$ this induced action is given as follows:

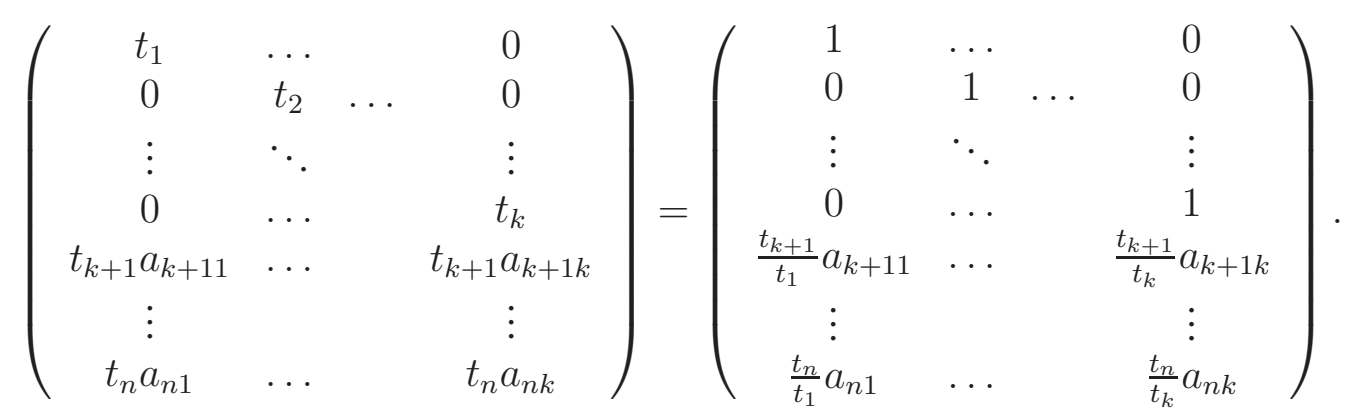

Let us consider a representation $\left(\mathbb{C}^{*}\right)^{n} \rightarrow\left(\mathbb{C}^{*}\right)^{k(n-k)}$ defined by

$$
\left(t_{1}, \ldots, t_{n}\right) \rightarrow\left(\frac{t_{k+1}}{t_{1}}, \ldots, \frac{t_{k+1}}{t_{k}}, \ldots, \frac{t_{n}}{t_{1}}, \ldots, \frac{t_{n}}{t_{k}}\right) .
$$

Put $\tau_{i}=\frac{t_{k+1}}{t_{i}}$ for $1 \leq i \leq k$ and $\tau_{k+i}=\frac{t_{k+i+1}}{t_{1}}$ for $1 \leq i \leq n-k-1$. It holds $\frac{t_{p}}{t_{s}}=\frac{\tau_{p-1} \tau_{s}}{\tau_{1}}$ for any $k+2 \leq p \leq n$ and $1 \leq s \leq k$. In this way, the representation of the torus $\left(\mathbb{C}^{*}\right)^{n}$ in $\left(\mathbb{C}^{*}\right)^{k(n-k)}$ is given by:

$$
\left(\tau_{1}, \ldots, \tau_{n}\right) \rightarrow\left(\tau_{1}, \ldots, \tau_{k}, \tau_{k+1}, \frac{\tau_{k+1} \tau_{2}}{\tau_{1}}, \ldots, \frac{\tau_{k+1} \tau_{k}}{\tau_{1}}, \tau_{k+2}, \frac{\tau_{k+2} \tau_{2}}{\tau_{1}}, \ldots \frac{\tau_{k+2} \tau_{k}}{\tau_{1}}, \ldots, \tau_{n}, \frac{\tau_{2} \tau_{n}}{\tau_{1}}, \ldots \frac{\tau_{n} \tau_{k}}{\tau_{1}}\right) .
$$

It follows that the action of the torus $\left(\mathbb{C}^{*}\right)^{n}$ on $\mathbb{C}^{k(n-k)}$ in a local chart for $G_{n, k}$ is given as a composition of the representation (6) and the standard action of the torus $\left(\mathbb{C}^{*}\right)^{k(n-k)}$ on $\mathbb{C}^{k(n-k)}$.

3.1. Orbits of $\left(\mathbb{C}^{*}\right)^{k}$-action on $\mathbb{C}^{n}$. In order to describe the action of the algebraic torus $\left(\mathbb{C}^{*}\right)^{n}$ locally in the charts for $G_{n, k}$ we consider more general situation.

Assume it is given an action of the torus $\left(\mathbb{C}^{*}\right)^{k}$ on $\mathbb{C}^{n}$ as a composition of a representation $\rho^{*}:\left(\mathbb{C}^{*}\right)^{k} \rightarrow\left(\mathbb{C}^{*}\right)^{n}$ and the standard action of $\left(\mathbb{C}^{*}\right)^{n}$ on $\mathbb{C}^{n}$. The representation $\rho^{*}$ induces the representation $\rho: T^{k} \rightarrow T^{n}$ which can be written as $\rho=\left(\rho_{1}, \ldots, \rho_{n}\right)$, where $\rho_{j}: T^{k} \rightarrow S^{1}$, $1 \leq j \leq n$ are homomorphisms. The characters $\rho_{j}$ can be further written as $\rho_{j}\left(t_{1}, \ldots, t_{k}\right)=$ $\rho_{j}\left(e^{2 \pi \sqrt{-1} x_{1}}, \ldots, e^{2 \pi \sqrt{-1} x_{k}}\right)=e^{2 \pi \sqrt{-1} \sum_{i=1}^{k} \alpha_{j}^{i} x_{i}}$ for some $\left(\alpha_{j}^{1}, \ldots, \alpha_{j}^{k}\right) \in \mathbb{Z}^{k}$. The vectors $\Lambda^{j}=$ $\left(\alpha_{j}^{1}, \ldots, \alpha_{j}^{k}\right), 1 \leq j \leq n$ are known to be the weight vectors of the representations $\rho$ and $\rho^{*}$.

Let us fix some subset $J=\left\{j_{1}, \ldots, j_{l}\right\} \subseteq\{1, \ldots, n\}$ and put

$$
\mathbb{C}^{J}=\left\{\left(z_{1}, \ldots, z_{n}\right) \in \mathbb{C}^{n} \mid z_{j} \neq 0, j \in J, z_{j}=0, j \notin J\right\} .
$$

The coordinate subspaces $\mathbb{C}^{J}$ are $\left(\mathbb{C}^{*}\right)^{k}$-invariant and $\mathbb{C}^{n}=\cup_{J} \mathbb{C}^{J}$. 
Denote by $V^{J}$ a matrix given by the weight vectors $\Lambda^{j}, j \in J$. This matrix defines a linear map $f_{J}: \mathbb{R}^{k} \rightarrow \mathbb{R}^{|J|}$ such that $f_{J}\left(\mathbb{Z}^{k}\right)$ is a direct summand in $\mathbb{Z}^{|J|}$. Let $q=\operatorname{rank} V^{J}$.

Lemma 3.1. The points from $\mathbb{C}^{J}$ have the same stabilizer $\left(\mathbb{C}^{*}\right)_{J}^{k} \subseteq\left(\mathbb{C}^{*}\right)^{k}$, where $\left(\mathbb{C}^{*}\right)_{J}^{k}=\left(\mathbb{C}^{*}\right)^{k-q}$, that is $\left(\mathbb{C}^{*}\right)^{q}$ acts freely on $\mathbb{C}^{J}$.

Proof. The stabilizer of a point $z \in \mathbb{C}^{J}$ is given by the intersection of the kernels of the characters $\rho_{j}, j \in J$. It is a subgroup $\left(\mathbb{C}^{*}\right)_{J}^{k} \subseteq\left(\mathbb{C}^{*}\right)^{k}$, and since we assume that $q=\operatorname{rank} V^{J}$, we obtain that $\left(\mathbb{C}^{*}\right)_{J}^{k}=\left(\mathbb{C}^{*}\right)^{k-q}$.

Corollary 3.2. If $l=|J|=q$, then $\mathbb{C}^{J}$ is an $\mathbb{C}^{k}$-orbit.

Let us consider now the case when $q<l=|J|$. We apply standard procedure for the description of the $\left(\mathbb{C}^{*}\right)^{k}$-orbit of a point from $\mathbb{C}^{J}$.

- Let $L$ be a lattice in $\mathbb{Z}^{l}$ spanned by $f_{J}\left(\Lambda^{j}\right), j \in J$.

- There exists a unique integral lattice $\hat{L}$ in $\mathbb{Z}^{l}$ which is orthogonal to $L$.

- By fixing a basis in $\hat{L}$ we obtain the matrix $\hat{V}^{J}$ of the dimension $l \times(l-q)$. Denote its elements by $\omega_{i}^{j} \in \mathbb{Z}, 1 \leq i \leq l, 1 \leq j \leq l-q$.

Let us consider an algebraic map $F_{J}:\left(\mathbb{C}^{*}\right)^{J} \rightarrow\left(\mathbb{C}^{*}\right)^{l-q}$ given by

$$
\left(z_{j_{1}}, \ldots, z_{j_{l}}\right) \rightarrow\left(z_{j_{1}}^{\omega_{1}^{1}} \ldots z_{j_{l}}^{\omega_{l}^{1}}, \ldots, z_{j_{1}}^{\omega_{1}^{l-q}} \cdots z_{j_{l}}^{\omega_{l}^{l-q}}\right) .
$$

Since the lattice $\hat{L}$ is orthogonal to $L$ we obtain:

Lemma 3.3. An algebraic map $F_{J}:\left(\mathbb{C}^{*}\right)^{J} \rightarrow\left(\mathbb{C}^{*}\right)^{l-q}$ is $\left(\mathbb{C}^{*}\right)^{k}$-invariant, where $\mathbb{C}^{l-q}$ is considered with trivial $\left(\mathbb{C}^{*}\right)^{k}$-action.

Corollary 3.4. The set of preimages $F_{J}^{-1}(c)$, where $c=\left(c_{1}, \ldots, c_{l-q}\right) \in\left(\mathbb{C}^{*}\right)^{l-q}$ gives the set of all $\left(\mathbb{C}^{*}\right)^{k}$-orbits in $\mathbb{C}^{J}$.

The characters of the representation $\rho: T^{n} \rightarrow T^{k(n-k)}$ which is given by (6), are:

$$
\begin{gathered}
\rho_{k+1, i}\left(\tau_{1}, \ldots, \tau_{n}\right)=\tau_{i}, \quad 1 \leq i \leq k, \\
\rho_{i, 1}\left(\tau_{1}, \ldots, \tau_{n}\right)=\tau_{i}, \quad k+1 \leq i \leq n,
\end{gathered}
$$

while for $\{p, s\} \neq\{k+1, i\},\{i, 1\}$, we obtain

$$
\rho_{p, s}=\frac{\tau_{p-1} \tau_{s}}{\tau_{1}}, 2 \leq s \leq k, k+2 \leq p \leq n .
$$

It follows that the weight vectors for the representation $\rho: T^{n} \rightarrow T^{k(n-k)}$ are given by

$$
\begin{gathered}
\Lambda_{k+1, i}=(0, \ldots 0, \underbrace{1}_{i}, 0, \ldots, 0), \quad 1 \leq i \leq k, \\
\Lambda_{i, 1}=(0, \ldots, 0, \underbrace{1}_{i}, 0, \ldots, 0), \quad k+1 \leq i \leq n, \\
\Lambda_{p, s}=(-1,0 \ldots, 0, \underbrace{1}_{p-1}, 0, \ldots, 0, \underbrace{1}_{s}, 0, \ldots, 0), \quad\{p, s\} \neq\{k+1, l\},\{l, 1\} .
\end{gathered}
$$

Let us consider a stratum $W_{\sigma}$ and assume that $W_{\sigma} \subset M_{I}$, where without loss of generality we can take that $I=\{1, \ldots, k\}$. We obtain the coordinate map $u_{I}: W_{\sigma} \rightarrow \mathbb{C}^{k(n-k)}$ given by $u_{I}(X)=\left(z_{1}, \ldots, z_{k(n-k)}\right)$. Note first the following: if $z_{i}=0$ for some $X_{0} \in W_{\sigma}$ then $z_{i}=0$ for all $X \in W_{\sigma}$. Namely, in the notation of Subsection 2.4, let $z_{i}=a_{p s}$ for some $k+1 \leq p \leq n$ and 
$1 \leq s \leq k$. Put $\hat{I}=(I-\{s\}) \cup\{p\}$. Then $P^{\hat{I}}\left(X_{0}\right)=0$, so the definition of $W_{\sigma}$ implies that $P^{\hat{I}}(X)=0$ for all $X \in W_{\sigma}$. This further implies that $z_{i}=0$ for all $X \in W_{\sigma}$.

Therefore, we may assume that

$$
u_{I}\left(W_{\sigma}\right) \subseteq \mathbb{C}^{J}=\left\{\left(z_{1}, \ldots, z_{k(n-k)}\right) \in \mathbb{C}^{k(n-k)}, z_{i} \neq 0, i \in J, z_{i}=0, i \notin J\right\} .
$$

Lemma 3.1 implies:

Proposition 3.5. All points of a stratum $W_{\sigma}$ in $G_{n, k}$ have the same stabilizer $\mathbb{C}_{\sigma}^{*} \subset\left(\mathbb{C}^{*}\right)^{n}$.

A stratum $W_{\sigma}$ is by definition invariant for the action of the algebraic torus $\left(\mathbb{C}^{*}\right)^{k}$. Denote by $I_{j_{1}}, \ldots, I_{j_{d}} \subset\{1, \ldots, k+1\},\left|I_{j_{i}}\right|=k$ such subsets that $P^{I_{j_{i}}}(X)=0$ for all $X \in W_{\sigma}$. Note that the conditions $P^{I_{j_{i}}}(X)=0$ impose relations on $\left(z_{1}, \ldots, z_{k(n-k)}\right) \in u_{I}\left(W_{\sigma}\right)$, which we denote by $P_{J}^{I_{i}}\left(z_{1}, \ldots, z_{k(n-k)}\right)=0$.

It follows that $u_{I}\left(W_{\sigma}\right)$ is:

- either the whole $\mathbb{C}^{J}$,

- or the intersection of the set of all $\left(\mathbb{C}^{*}\right)^{k}$-orbits in $\mathbb{C}^{J}$ with the surfaces which are defined by the equations imposed by those Plücker coordinates which are zero for the points of $W_{\sigma}$ :

$$
u_{I}\left(W_{\sigma}\right)=\left\{\begin{array}{l}
F_{J}^{-1}(c), c=\left(c_{1}, \ldots, c_{l-q}\right) \in\left(\mathbb{C}^{*}\right)^{l-q} \\
P_{J}^{I_{i}}\left(z_{1}, \ldots, z_{k(n-k)}\right)=0, i=1, \ldots, d .
\end{array}\right.
$$

Here $l$ and $q$ are as in the previous paragraph.

As a result we obtain a family of algebraic surfaces which are parametrized by $F_{\sigma, I} \subseteq\left(\mathbb{C}^{*}\right)^{l-q}$. Here $F_{\sigma, I}=\left\{c \in\left(\mathbb{C}^{*}\right)^{l-1} \mid F_{J}^{-1}(c) \cap\left\{\left(z_{1}, \ldots, z_{k(n-k)}\right) \mid P_{J}^{I_{i}}\left(z_{1}, \ldots, z_{k(n-k)}\right)=0, i=1, \ldots, d\right\} \neq\right.$ $\emptyset\}$ is an open algebraic manifold. Any of these surfaces is, by Lemma 3.1, an $\left(\mathbb{C}^{*}\right)^{q}$-orbit. According to [1], this orbit maps by the moment map to $\stackrel{\circ}{P}_{\sigma}$. In this way, we obtain that $W_{\sigma} /\left(\mathbb{C}^{*}\right)^{n}=F_{\sigma}$ is homeomorphic to $F_{\sigma, I}$ and that the map $\widehat{\mu}: W_{\sigma} / T^{n} \rightarrow \stackrel{\circ}{P_{\sigma}}$ is a fiber bundle with the fiber either a point either $F_{\sigma, I} \subseteq\left(\mathbb{C}^{*}\right)^{l-q}$.

Thus, we proved:

Theorem 3.6. The map $\hat{\mu}: W_{\sigma} / T^{n} \rightarrow \stackrel{\circ}{P}_{\sigma}$ is a locally trivial fiber bundle whose fiber is an open algebraic manifold $F_{\sigma}$. Thus, $W_{\sigma} / T^{n} \cong \stackrel{\circ}{P}_{\sigma} \times F_{\sigma}$.

We want to emphasize that the trivialization stated in Theorem 3.6 is canonical:

Proposition 3.7. There is the canonical trivialization $h_{\sigma}: W_{\sigma} / T^{n} \rightarrow \stackrel{\circ}{P_{\sigma}} \times F_{\sigma}$.

Proof. The space of parameters $F_{\sigma}$ is defined by $F_{\sigma}=W_{\sigma} /\left(\mathbb{C}^{*}\right)^{n}$, so denote by $p_{\sigma}: W_{\sigma} / T^{n} \rightarrow F_{\sigma}$ the canonical projection. It follows that the map $h_{\sigma}: W_{\sigma} / T^{n} \rightarrow \stackrel{\circ}{P_{\sigma}} \times F_{\sigma}$ defined by $h_{\sigma}=\left(\hat{\mu}, p_{\sigma}\right)$ is a canonical homeomorphism.

We use the notation $h: W / T^{n} \rightarrow \Delta_{n, k}^{\circ} \times F$ for the canonical trivialization of the main stratum.

3.2. On singular and regular values of the moment map. . Let us consider an effective action of the algebraic torus $\left(\mathbb{C}^{*}\right)^{n-1}$ on $G_{n, k}$. We want to prove that a regular point of the moment map $\mu: G_{n, k} \rightarrow \Delta_{n, k}$ is determined by the non-triviality of its stationary subgroup. In order to do that we consider more general situation. 
Let us consider the complex projective space $\mathbb{C} P^{N}$, where $N=\left(\begin{array}{l}n \\ k\end{array}\right)-1$ endowed with an action of the torus $T^{n}$ which is given by a composition of the $k$-th exterior power representation $\rho: T^{n} \rightarrow T^{N+1}$ and the canonical action of $T^{N+1}$ on $\mathbb{C} P^{N}$. This action extends to an action of the algebraic torus $\left(\mathbb{C}^{*}\right)^{n}$. Let $\Lambda_{I} \in \mathbb{Z}^{n}$ denote the weight vectors of the representation $\rho$, where $I \in\left(\begin{array}{l}n \\ k\end{array}\right)$. More precisely $\Lambda_{I}^{i}=1$ for $i \in I$, while $\Lambda_{I}^{i}=0$ for $i \notin I$. Then the moment map $\mu: \mathbb{C} P^{N} \rightarrow \mathbb{R}^{n}$ for the considered $T^{n}$ action on $\mathbb{C} P^{N}$ is given by

$$
\mu\left(\left[z_{I}, I \in\left(\begin{array}{l}
n \\
k
\end{array}\right)\right]\right)=\frac{1}{\sum_{I \in\left(\begin{array}{l}
n \\
k
\end{array}\right)}\left|z_{I}\right|^{2}} \sum_{I \in\left(\begin{array}{l}
n \\
k
\end{array}\right)}\left|z_{I}\right|^{2} \Lambda_{I}
$$

We want to determine the rank of the differential $d \mu$ at an arbitrary point $\mathbf{z}=\left[z_{I}\right] \in \mathbb{C} P^{N}$. Let $P_{\mathbf{z}}$ denote a polytope which is the convex span of those vectors $\Lambda_{I}$ such that $z_{I} \neq 0$.

Theorem 3.8. The rank of the differential of the moment map $\mu: \mathbb{C} P^{N} \rightarrow \mathbb{R}^{n}$ given by (9) is

$$
\operatorname{rk} d \mu(\mathbf{z})=\operatorname{dim} P_{\mathbf{z}}
$$

for an arbitrary point $\mathbf{z} \in \mathbb{C} P^{N}$.

Proof. We first note that the map $\mu$ is $T^{n}$-invariant and that the image of $\mu$ is the hypersimplex $\Delta_{n, k}$. This implies that $\mathrm{rk} d \mu \leq n-1$ for any $z \in \mathbb{C} P^{N}$. We also note that the map $\mu: \mathrm{O}(\mathbf{z}) \rightarrow \stackrel{\circ}{P}_{\mathbf{z}}$ is a smooth fiber bundle for the $\left(\mathbb{C}^{*}\right)^{n}$-orbit $\mathrm{O}(\mathbf{z})$ of a point $\mathbf{z}$, where $\stackrel{\circ}{P_{\mathbf{z}}}$ is an interior of the polytope $P_{\mathbf{z}}$. It implies that the rank of the differential $d \mu$ at a point $\mathbf{z}$ is $\geq \operatorname{dim} P_{\mathbf{z}}$. Therefore, we differentiate the following cases for the point $\mathrm{z}$ :

- If $z_{I} \neq 0$ for all $I \in\left(\begin{array}{l}n \\ k\end{array}\right)$ then $\stackrel{\circ}{P}_{\mathbf{z}}=\stackrel{\circ}{\Delta}_{n, k}$ and $\operatorname{rk} d \mu(\mathbf{z})=n-1$.

- Let $\left\{I_{1}, \ldots, I_{l}\right\}$ denote the set of those $I$ for which $z_{I_{j}}=0$, while $z_{I} \neq 0$ for $I \notin$ $\left\{I_{1}, \ldots I_{l}\right\}$. Fix a chart in $\mathbb{C} P^{N}$ determined by the condition that the coordinate indexed by a fixed $I_{p} \notin\left\{I_{1}, \ldots I_{l}\right\}$ is not zero. Then the point $\mathbf{z}$ belongs to this chart and the moment map $\mu$, in local coordinates of this chart, is given by

$$
\mu\left(\left[z_{I}, I \in\left(\begin{array}{l}
n \\
k
\end{array}\right) \backslash I_{p}\right]\right)=\frac{1}{1+\sum_{I \neq I_{p}}\left|z_{I}\right|^{2}}\left(\Lambda_{I_{p}}+\sum_{I \neq I_{p}}\left|z_{I}\right|^{2} \Lambda_{I}\right) .
$$

Let $z_{I}=x_{I}+i y_{I}$. It follows that

$$
\frac{\partial \mu}{\partial x_{I_{0}}}=\frac{1}{\left(1+\sum_{I \neq I_{p}}\left|z_{I}\right|^{2}\right)^{2}}\left(2 x_{I_{0}}\left(1+\sum_{I_{\neq} I_{p}}\left|z_{I}\right|^{2}\right) \Lambda_{I_{0}}-2 x_{I_{0}}\left(\Lambda_{I_{p}}+\sum_{I \neq I_{p}}\left|z_{I}\right|^{2} \Lambda_{I}\right)\right)
$$

and

$$
\frac{\partial \mu}{\partial y_{I_{0}}}=\frac{1}{\left(1+\sum_{I \neq I_{p}}\left|z_{I}\right|^{2}\right)^{2}}\left(2 y_{I_{0}}\left(1+\sum_{I_{\mp} I_{p}}\left|z_{I}\right|^{2}\right) \Lambda_{I_{0}}-2 y_{I_{0}}\left(\Lambda_{I_{p}}+\sum_{I \neq I_{p}}\left|z_{I}\right|^{2} \Lambda_{I}\right)\right)
$$

Therefore, $\frac{\partial \mu}{\partial x_{I_{0}}}=\frac{\partial \mu}{\partial I_{0}}=0$ at a point $z_{I_{0}}=0$. It implies that $\mathrm{rk} d \mu$ at a point $z_{I_{0}}=0$ is equal to the $\left.\operatorname{rk} d \mu\right|_{z_{I_{0}}=0}$, where $\left.\mu\right|_{z_{I_{0}}=0}$ is the restriction of $\mu$ to the coordinate subspace $z_{I_{0}}=0$, that is

$$
(r k d \mu)\left(z_{I_{0}}=0\right)=\left(\operatorname{rkd} d\left(\mu \mid z_{I_{0}}=0\right)\right)\left(z_{I, I \neq I_{0}}\right),
$$


Altogether we obtain that

$$
(\operatorname{rank} d \mu)(\mathbf{z})=\operatorname{rank} d\left(\left.\mu\right|_{z_{I_{1}}=\ldots=z_{I_{l}}=0}\right)\left(z_{I, I \neq I_{1}, \ldots I_{l}}\right) .
$$

Denote by $\mathbb{C} P_{I_{1}, \ldots I_{l}}^{N-l}$ the complex projective space defined by the coordinate subspace $\mathbb{C}_{I_{1}, \ldots \ldots I_{l}}^{N-l+1}$ in $\mathbb{C}^{N+1}$ whose axis are indexed by $I \notin\left\{I_{1}, \ldots I_{l}\right\}$ and denote by $P_{I_{1}, \ldots, I_{l}}$ the convex polytope which is obtained as the convex hull of the vectors $\Lambda_{I}, I \notin\left\{I_{1}, \ldots, I_{l}\right\}$. Let us consider the map

$$
\left.\mu\right|_{z_{I_{1}}=\ldots=z_{I_{l}}=0}: \mathbb{C} P_{I_{1}, \ldots, I_{l}}^{N-l} \rightarrow P_{I_{1}, \ldots I_{l}} .
$$

The point $\mathbf{z}$ belongs to $\mathbb{C} P_{I_{1}, \ldots I_{l}}^{N-l}$ as well as its $\left(\mathbb{C}^{*}\right)_{I_{1}, \ldots, I_{l}}^{N-l+1}$-orbit. Since all coordinates for $\mathbf{z} \in \mathbb{C} P_{I_{1}, \ldots I_{l}}^{N-l}$ are non zero, it follows that

$$
\left.\left.\mu\right|_{\mathbb{C} P_{I_{1}, \ldots I_{l}}^{N-l}}\left(\left(\mathbb{C}^{*}\right)_{I_{1}, \ldots, I_{l}}^{N-l+1} \cdot \mathbf{z}\right)\right)=\stackrel{\circ}{P}{ }_{I_{1}, \ldots I_{l}} .
$$

Thus, $P_{\mathbf{z}}=P_{I_{1}, \ldots I_{l}}$ and

$$
\operatorname{rk} d\left(\left.\mu\right|_{\mathbb{C} P_{I_{1}, \ldots I_{l}}^{N-l}}\right)(\mathbf{z})=\operatorname{dim} P_{I_{1}, \ldots, I_{l}} .
$$

Together with (11) this proves that $(\operatorname{rk} d \mu)(\mathbf{z})=\operatorname{dim} P_{\mathbf{z}}$.

By Lemma 2.6 the Plücker embedding $G_{n, k} \rightarrow \mathbb{C} P^{N}$ is $T^{n}$-equivariant. Therefore, by Theorem 3.8 for the moment map $\mu: G_{n, k} \rightarrow \mathbb{R}^{n}$ defined by (2) it holds:

Corollary 3.9. The rank of the differential of the moment map $\mu: G_{n, k} \rightarrow \mathbb{R}^{n}$ is given by

$$
\operatorname{rk} d \mu(L)=\operatorname{dim} P_{L}
$$

at an arbitrary point $L$, where $P_{L}$ is an admissible polytope for the point $L \in G_{n, k}$, that is $P_{L}=$ $\mu\left(\overline{\left(\mathbb{C}^{*}\right)^{n} \cdot L}\right)$.

Together with Theorem 2.15 this further gives:

Corollary 3.10. A point $L \in G_{n, k}$ is a regular point for the moment map $\mu: G_{n, k} \rightarrow \mathbb{R}^{n}$ if and only if its stationary subgroup $\mathbb{C}_{L}^{*} \subset\left(\mathbb{C}^{*}\right)^{n+1}$ is trivial.

It follows that the singular points in $G_{n, k}$ are given by those strata $W_{\sigma}$ whose stationary subgroups $\mathbb{C}_{\sigma}^{*}$ are non-trivial.

As for the regular values of the moment map we deduce:

Theorem 3.11. A point $x \in \stackrel{\circ}{\Delta}_{n, k}$ is a regular value for the moment map $\mu: G_{n, k} \rightarrow \Delta_{n, k}$ if and only if the preimage $\mu^{-1}(x)$ does not intersect any stratum which has non-trivial stationary subgroup.

Remark 3.12. According to classical results of mathematical analysis, $\mu^{-1}(x)$ is a closed smooth submanifold in $G_{n, k}$ of dimension $q+n-1$ for a regular value $x \in \stackrel{\circ}{\Delta}, k_{n}$. Here $q=2(k-1)(n-k-1)$ is the dimension of the space of parameters of the main stratum.

We define the defect of a point $L \in G_{n, k}$ to be an integer $q$ such that the $T^{n}$ - orbit of $L$ has the dimension $n-1-q$. Then Theorem 3.11 can be reformulated as:

Corollary 3.13. A point $x \in \stackrel{\circ}{\Delta}_{n, k}$ is a regular value for the moment map $\mu: G_{n, k} \rightarrow \Delta_{n, k}$ if and only if any point from the preimage $\mu^{-1}(x)$ has the defect equal to zero. 
We provide geometrical description of some submanifolds in $G_{n, k}$, which consist of singular points for the moment map $\mu$. Let us consider the decomposition $\mathbb{C}^{n}=\mathbb{C}^{n_{1}} \times \mathbb{C}^{n_{2}}, n=n_{1}+n_{2}$, and consider the submanifold $G_{n_{1}, k_{1}, n_{2}, k_{2}}, k=k_{1}+k_{2}$, in $G_{n, k}$, which consists of all $k$-dimensional subspaces $L \subset \mathbb{C}^{n}$ such that $L=\mathcal{L}\left(L_{1}, L_{2}\right)$, where $L_{1}$ is a $k_{1}$-dimensional subspace in $\mathbb{C}^{n_{1}}$ and $L_{2}$ is a $k_{2}$-dimensional subspace in $\mathbb{C}^{n_{2}}$. Then $G_{n_{1}, k_{1}, n_{2}, k_{1}} \cong G_{n_{1}, k_{1}} \times G_{n_{2}, k_{2}}$ and the action of $\left(\mathbb{C}^{*}\right)^{n}$ on $G_{n_{1}, k_{1}, n_{2}, k_{2}}$ is induced by the canonical action of $\left(\mathbb{C}^{*}\right)^{n_{1}}$ on $G_{n_{1}, k_{1}}$ and the canonical action of $\left(\mathbb{C}^{*}\right)^{n_{2}}$ on $G_{n_{2}, k_{2}}$. Therefore, the defect of a point $L \in G_{n_{1}, k_{1}, n_{2}, k_{2}}$ is $q \geq 1$. Then Corollary 3.13 implies:

Proposition 3.14. The points which belong to the submanifolds $G_{n_{1}, k_{1}, n_{2}, k_{2}} \cong G_{n_{1}, k_{1}} \times G_{n_{2}, k_{2}}$ in $G_{n, k}$ are singular points for the moment map $\mu: G_{n, k} \rightarrow \Delta_{n, k}$.

Consider a function $f_{j}: \Delta_{n, k} \rightarrow \mathbb{R}$ defined by the projection on the $j$-th coordinate, that is $f\left(x_{1}, \ldots, x_{n}\right)=x_{j}$, where $1 \leq j \leq n$ and the function $\mu_{j}: G_{n, k} \rightarrow \mathbb{R}, 1 \leq j \leq n$ defined by the composition $\mu_{j}=f_{j} \circ \mu$.

Lemma 3.15. The set of critical values of the function $\mu_{j}$ consists of two points 0 and 1 , and the set of its critical points is a disjoint union of the smooth manifolds $\mu_{j}^{-1}(0)=G_{n-1, k}$ and $\mu_{j}^{-1}(1)=G_{n-1, k-1}$.

Proof. Note that $\mu_{j}\left(G_{n, k}\right)=[0,1]$. By Corollary 3.9 we have that any fixed point $L$ for the considered $T^{n}$-action is a critical point for the map $\mu_{j}$. Since $\mu_{j}(L)$ is equal to 0 or 1 for any fixed point $L$, it follows that the points 0 and 1 are critical values for $\mu_{j}$. It is easy to deduce from the proof of Corollary 3.9 that no $t \in(0,1)$ can be a critical value for $\mu_{j}$. Moreover, $\mu_{j}^{-1}(0) \cong G_{n-1, k}$ while $\mu_{j}^{-1}(1) \cong G_{n-1, k-1}$.

Note that since the differential of the map $\mu_{j}: G_{n, k} \backslash\left(G_{n-1, k} \cup G_{n-1, k-1}\right) \rightarrow(0,1)$ is an epimorphism it follows that there exists a smooth, closed $T^{n}$ - invariant submanifold $S_{n, k}$ in $G_{n, k}$ such that $G_{n, k} \backslash\left(G_{n-1, k} \cup G_{n-1, k-1}\right) \cong S_{n, k} \times(0,1)$. Altogether the following result holds.

Proposition 3.16. There exist $T^{n}$-equivariant projections $\pi_{0}: S_{n, k} \rightarrow G_{n-1, k}$ and $\pi_{1}: S_{n, k} \rightarrow$ $G_{n-1, k-1}$ such that

$$
G_{n, k}=\left(S_{n, k} \times[0,1]\right) \cup_{\pi_{0}} G_{n-1, k} \cup_{\pi_{1}} G_{n-1, k-1},
$$

where $(s, 0) \simeq \pi_{0}(s)$ and $(s, 1) \simeq \pi_{1}(s)$.

It immediately follows:

Theorem 3.17. There exist projections $\hat{\pi}_{0}: S_{n, k} / T^{n} \rightarrow G_{n-1, k} / T^{n-1}$ and $\hat{\pi}_{1}: S_{n, k} / T^{n} \rightarrow$ $G_{n-1, k-1} / T^{n-1}$ such that

$$
G_{n, k} / T^{n}=\left(S_{n, k} / T^{n} \times[0,1]\right) \cup_{\hat{\pi}_{0}} G_{n-1, k} / T^{n-1} \cup_{\hat{\pi}_{1}} G_{n-1, k-1} / T^{n-1},
$$

where $[(s, 0)] \simeq \hat{\pi}_{0}[s]$ and $[(s, 1)] \simeq \hat{\pi}_{1}[s]$.

For $k=2$ this yields to the following result.

Corollary 3.18. The orbit space $G_{n, 2} / T^{n}$ is homotopy equivalent to the space $C\left(S_{n, 2} / T^{n}\right) \cup_{\hat{\pi}_{0}}$ $G_{n-1,2} / T^{n-1}$ that is obtained by attaching the conus $C\left(S_{n, 2} / T^{n}\right)$ to the space $G_{n-1,2} / T^{n-1}$ by the attaching map $\hat{\pi}_{0}: S_{n, 2} / T^{n} \cong \partial C\left(S_{n, 2} / T^{n}\right) \rightarrow G_{n-1,2} / T^{n-1}$.

In this way we obtain an inductive description of the orbit space $G_{n, 2} / T^{n}$ in terms of the orbit spaces $S_{l, 2} / T^{l}$, where $l \leq n$. 
Example 3.19. For $n=4$ and $k=2$ we obtain that $\Sigma\left(S_{4,2} / T^{4}\right)$ is homotopy equivalent to $G_{4,2} / T^{4} \cong S^{5}$. For $n=5$ and $k=2$ we obtain that $G_{5,2} / T^{5}$ is homotopy equivalent to $C\left(S_{5,2} / T^{5}\right) \cup_{\hat{\pi}_{0}} S^{5}$.

Remark 3.20. It arises a problem to describe the orbit space $S_{n, k} / T^{n}$. This will be a topic of our forthcoming paper.

Remark 3.21. Note that the manifolds $G_{n_{1}, k_{1}, n_{2}, k_{2}}$ are the closures of the strata in $G_{n, k}$ which are mapped, by the moment map, to the polytopes $\Delta_{n_{1}, k_{1}} \times \Delta_{n_{2}, k_{2}} \subset \Delta_{n, k}$. The polytopes $\Delta_{n_{1}, k_{1}} \times$ $\Delta_{n_{2}, k_{2}}$ can be perceived as the intersection of $\Delta_{n, k}$ with the planes $x_{1}+\cdots+x_{n_{1}}=k_{1}$ in $\mathbb{R}^{n}$ according to the decomposition $\mathbb{R}^{n}=\mathbb{R}^{n_{1}} \times \mathbb{R}^{n_{2}}$, where $1 \leq k_{1} \leq n_{1}, 1 \leq n_{1} \leq n$.

\section{The CANONiCAL ACTION OF $\left(\mathbb{C}^{*}\right)^{5}$ ON $G_{5,2}$}

We consider the canonical action of $T^{5}$ on the complex Grassmann manifold $G_{5,2}$. Note that the diagonal $\Delta$ in $T^{5}$ acts trivially on $G_{5,2}$, so the torus $T^{4} / \Delta$ acts effectively on $G_{5,2}$. In the sequel we equivalently consider both of these actions. The image of $G_{5,2}$ by the moment map (2) is the convex hull of the points $\Lambda_{i j}=\left(\delta_{i j}^{l}\right) \in \mathbb{R}^{5}, 1 \leq l \leq 5$ and $1 \leq i<j \leq 5$, such that $\delta_{i j}^{i}=\delta_{i j}^{j}=1$ and $\delta_{i j}^{l}=0$ for $l \neq i, j$. This polytope is known as the hypersimplex $\Delta_{5,2}$. The hypersimplex $\Delta_{5,2}$ is a four-dimensional polytope which belongs to the hyperplane $x_{1}+\ldots+x_{5}=2$. The atlas for $G_{5,2}$, defined by the Plücker coordinates, consists of ten charts $M_{I}=\left\{L \in G_{5,2} \mid P^{I}(L) \neq 0\right\}$, where $I=\left\{i_{1}, i_{2}\right\} \subset\{1, \ldots, 5\}$. We describe the strata on $G_{5,2}$ following the pattern given in [7].

4.1. $\left(\mathbb{C}^{*}\right)^{5}$-orbits and their admissible polytopes. We first explicitly describe the orbits of the $\left(\mathbb{C}^{*}\right)^{5}$-action on $\mathbb{C}^{6}$ in a fixed chart.

Proposition 4.1. The orbit of a point $\mathbf{a}=\left(a_{1}, \ldots, a_{6}\right) \in \mathbb{C}^{6}$ for the induced $\left(\mathbb{C}^{*}\right)^{5}$-action on $\mathbb{C}^{6}$ is one of the following:

(1) it is the 8-dimensional manifold given by the equations

$c_{1}^{\prime} z_{1} z_{5}=c_{1} z_{2} z_{4}, \quad c_{2}^{\prime} z_{1} z_{6}=c_{2} z_{3} z_{4}, \quad c_{3}^{\prime} z_{2} z_{6}=c_{3} z_{3} z_{5}$, for $c_{i}, c_{i}^{\prime} \neq 0$ and $c_{1} c_{2}^{\prime} c_{3}=c_{1}^{\prime} c_{2} c_{3}^{\prime}$,

if $a_{i} \neq 0,1 \leq i \leq 6$;

(2) it is the 8-dimensional manifold given by one of the following six equations:

(a) $c_{1}^{\prime} z_{1} z_{5}=c_{1} z_{2} z_{4}, z_{3}=0$ or $z_{6}=0$ textfor $c_{1}, c_{1}^{\prime} \neq 0$, if $a_{3}=0$ or $a_{6}=0$,

(b) $c_{2}^{\prime} z_{1} z_{6}=c_{2} z_{3} z_{4}, z_{2}=0$ or $z_{5}=0$ for $c_{2}, c_{2}^{\prime} \neq 0$, if $a_{2}=0$ or $a_{5}=0$,

(c) $c_{3}^{\prime} z_{2} z_{6}=c_{3} z_{3} z_{5}, z_{1}=0$ or $z_{4}=0$ for $c_{3}, c_{3}^{\prime} \neq 0$, if $a_{1}=0$ or $a_{4}=0$;

(3) it is the 6-dimensional manifold given by one of the following three equations:

(a) $c_{1}^{\prime} z_{1} z_{5}=c_{1} z_{2} z_{4}, z_{3}=z_{6}=0$ for $c, c^{\prime} \neq 0$, if $a_{3}=a_{6}=0$,

(b) $c_{2}^{\prime} z_{1} z_{6}=c_{2} z_{3} z_{4}, z_{2}=z_{5}=0$ for $c, c^{\prime} \neq 0$, if $a_{2}=a_{5}=0$,

(c) $c_{3} z_{2} z_{6}=c_{3}^{\prime} z_{3} z_{5}, z_{1}=z_{4}=0$ for $c, c^{\prime} \neq 0$, if $a_{1}=a_{4}=0$;

(4) it is $\mathbb{C}_{I}^{*} \subset\left(\mathbb{C}^{*}\right)^{6}$, where $I \subset\{1, \ldots, 6\},|I|=4$ and $I \neq\{2,3,5,6\},\{1,3,4,6\},\{1,2,4,5\}$,

if $a_{i}=a_{j}=0, \quad\{i, j\} \neq\{1,4\},\{2,5\},\{3,6\}$;

(5) it is $\mathbb{C}_{I}^{*} \subset\left(\mathbb{C}^{*}\right)^{6}$, where $I \subset\{1, \ldots, 6\},|I| \leq 3$, if three of more coordinates of a are zero.

Proof. Without loss of generality we consider the chart $M_{12}$. It follows from (6) that the $\left(\mathbb{C}^{*}\right)^{5}$ orbit of a point $L \in M_{12}$ is given by $\left(t_{1} a_{1}, t_{2} a_{2}, t_{3} a_{3}, t_{4} a_{4}, \frac{t_{2} t_{4}}{t_{1}} a_{5}, \frac{t_{3} t_{4}}{t_{1}} a_{6}\right)$. Thus, if $a_{i} \neq 0$ for all $1 \leq i \leq 6$ then an element $\left(z_{1}, \ldots, z_{6}\right)$ belongs to this orbit if and only if it satisfies the system of equations

$$
c_{1}^{\prime} z_{1} z_{5}=c_{1} z_{2} z_{4}, \quad c_{2}^{\prime} z_{1} z_{6}=c_{2} z_{3} z_{4}
$$


where $c_{1}=a_{1} a_{5}, c_{1}^{\prime}=a_{2} a_{4}, c_{2}=a_{1} a_{6}, c_{2}^{\prime}=a_{3} a_{4}, c_{3}=a_{2} a_{6}, c_{3}^{\prime}=a_{3} a_{5}$. Since $c_{i}, c_{i}^{\prime} \neq 0$ we obtain in this way the orbits of the type (1).

If exactly one of the $a_{i}$ 's is equal to zero we obtain the orbits of the type (2). For example if $a_{1}$ or $a_{4}$ is equal to zero we obtain the orbits $(c)$ in $(2)$. The orbits of the type $(3)$ are obtained by elements $\left(a_{1}, \ldots, a_{6}\right)$ such that two of the $a_{i}$ 's which belong to the same row are equal to zero. For all other choices of an element $\left(a_{1}, \ldots, a_{6}\right) \in \mathbb{C}^{6}$, its orbit will be a coordinate subspace as stated in the Proposition.

If two of the $a_{i}$ 's which do not belong to the same row are equal to zero, say $a_{1}=a_{5}=0$, the orbit of a such element will be $\left(0, t_{2} a_{2}, t_{3} a_{3}, t_{4} a_{4}, 0, \frac{t_{3} t_{4}}{t_{1}} a_{6}\right)$. This orbits writes as $\left(0, t_{2} a_{2}, t_{3} a_{3}, t_{4} a_{4}, 0, t_{1} a_{6}\right)$. It implies that the orbit of this element is coordinate subspace $\mathbb{C}_{2346}^{*}$. Similarly, the complex fourdimensional coordinate subspaces $\mathbb{C}_{i j k l}^{*},\{i, j, k, l\} \neq\{1,3,4,6\},\{2,3,5,6\},\{1,2,4,5\}$ are 8dimensional orbits of the algebraic torus $\left(\mathbb{C}^{*}\right)^{5}$.

If three or more $a_{i}$ 's are equal to zero then it can be directly checked that the orbit of a such element will be $\mathbb{C}_{I}^{*}$, where $I=\left\{i \mid a_{i} \neq 0\right\}$. In this way, we obtain all possible $\mathbb{C}_{I}^{*}$, where $I \subset$ $\{1, \ldots, 6\}$ and $|I| \leq 3$.

4.2. Admissible polytopes in the chart $M_{12}$. The image by the moment map of an arbitrary $\left(\mathbb{C}^{*}\right)^{5}$-orbit is the interior of a convex polytope spanned by some subset of vertices for $\Delta_{5,2}$. These polytopes, as we already said, are called the admissible polytopes. We describe here admissible polytopes for $\left(\mathbb{C}^{*}\right)^{5}$-orbits, which belong to the chart $M_{12}$, following their description given in Proposition 4.1 .

Proposition 4.2. Admissible polytopes for $\left(\mathbb{C}^{*}\right)^{5}$ - orbits in $G_{5,2}$ in the chart $M_{12}$ are, according to Proposition 4.1] as follows:

(1) For the orbits (1) - one of the following four-dimensional polytopes:

- the hypersimplex $\Delta_{5,2}$ if $c_{i}, c_{i}^{\prime} \neq 0, c_{i} \neq c_{i}^{\prime}$;

- spanned by 9 vertices different from $\Lambda_{45}$ if $c_{1} \neq c_{1}^{\prime}, c_{3}=c_{3}^{\prime}$;

- spanned by 9 vertices different from $\Lambda_{35}$ if $c_{1} \neq c_{1}^{\prime}, c_{2}=c_{2}^{\prime}$;

- spanned by 9 vertices different $\Lambda_{34}$ if $c_{1}=c_{1}^{\prime}, c_{2} \neq c_{2}^{\prime}$;

- spanned by 7 vertices different from $\Lambda_{34}, \Lambda_{35}, \Lambda_{45}$ if $c_{1}=c_{1}^{\prime} ; c_{2}=c_{2}^{\prime}, c_{3}=c_{3}^{\prime}$.

(2) For the orbits (2) - one of the following four-dimensional polytopes:

(a) spanned by 9 vertices different from:

- $\Lambda_{25}$ or $\Lambda_{15}$ for the orbits (a),

- $\Lambda_{24}$ or $\Lambda_{14}$ for the orbits (b),

- $\Lambda_{23}$ or $\Lambda_{13}$ for the orbits (c)

if $c_{i} \neq c_{i}^{\prime}$;

(b) spanned by 8 vertices different from:

- $\Lambda_{34}, \Lambda_{25}$ or $\Lambda_{34}, \Lambda_{15}$ for the orbits (a),

- $\Lambda_{35}, \Lambda_{24}$ or $\Lambda_{35}, \Lambda_{14}$ for the orbits $(b)$,

- $\Lambda_{45}, \Lambda_{23}$ or $\Lambda_{45}, \Lambda_{13}$ for the orbits (c)

if $c_{i}=c_{i}^{\prime}$.

(3) For the orbits (3) - one of the following three-dimensional polytopes:

(a) an octahedron spanned by the vertices:

$$
\begin{aligned}
& \Lambda_{12}, \Lambda_{13}, \Lambda_{14}, \Lambda_{23}, \Lambda_{24}, \Lambda_{34} ; \\
& \Lambda_{12}, \Lambda_{13}, \Lambda_{15}, \Lambda_{23}, \Lambda_{25}, \Lambda_{35}
\end{aligned}
$$




$$
\Lambda_{12}, \Lambda_{14}, \Lambda_{15}, \Lambda_{24}, \Lambda_{25}, \Lambda_{45}
$$

if $c_{i} \neq c_{i}^{\prime}$;

(b) spanned by the vertices:

- $\Lambda_{12}, \Lambda_{13}, \Lambda_{14}, \Lambda_{23}, \Lambda_{24}$ for the orbits (a),

- $\Lambda_{12}, \Lambda_{13}, \Lambda_{15}, \Lambda_{23}, \Lambda_{25}$ for the orbits $(b)$,

- $\Lambda_{12}, \Lambda_{14}, \Lambda_{15}, \Lambda_{24}, \Lambda_{25}$ for the orbits (c)

if $c_{i}=c_{i}^{\prime}$.

(4) For the orbits (4) - one of the following four-dimensional polytopes:

- spanned by 8 vertices, which do not contain the pairs $\Lambda_{1 i}, \Lambda_{2 j}$, where $i \neq j$ and $i, j \geq 3$

- spanned by 7 vertices, which do not contain the triples $\Lambda_{1 i}, \Lambda_{1 j}, \Lambda_{i j}$ or the pairs $\Lambda_{2 i}, \Lambda_{2 j}$ where $i \neq j$ and $i, j \geq 3$;

(5) For the orbits (5) - one of the following one, two or three-dimensional polytopes:

- spanned by $\Lambda_{12}, \Lambda_{1 i}$ or $\Lambda_{12}, \Lambda_{2 i}$, where $3 \leq i \leq 5$ for the one-dimensional polytopes;

- spanned by $\Lambda_{12}, \Lambda_{1 i}, \Lambda_{1 j}$ or $\Lambda_{12}, \Lambda_{2 i}, \Lambda_{2 j}$ or $\Lambda_{12}, \Lambda_{1 i}, \Lambda_{2 i}$ or $\Lambda_{12}, \Lambda_{1 i}, \Lambda_{2 j}, \Lambda_{i j}$, where $3 \leq i, j \leq 5$ and $i \neq j$ for the two-dimensional polytopes;

- spanned by $\Lambda_{12}, \Lambda_{13}, \Lambda_{14}, \Lambda_{15}$ or $\Lambda_{12}, \Lambda_{23}, \Lambda_{24} \Lambda_{25}$ or $\Lambda_{12}, \Lambda_{1 i}, \Lambda_{1 j}, \Lambda_{2 i}, \Lambda_{i j}$ or $\Lambda_{12}$, $\Lambda_{2 i}, \Lambda_{2 j}, \Lambda_{1 i}, \Lambda_{i j}$ or $\Lambda_{12}, \Lambda_{1 i}, \Lambda_{1 j}, \Lambda_{2 k}, \Lambda_{i k}, \Lambda_{j k}$ or $\Lambda_{12}, \Lambda_{2 i}, \Lambda_{2 j}, \Lambda_{1 k}, \Lambda_{i k}, \Lambda_{j k}$ where $i, j, k \geq 3, i \neq j, k$ and $k \neq j$, for the three-dimensional polytopes.

Proof. The proof is similar to the proof of Proposition 4.1. For the orbits of type (1) such that $c_{1} \neq c_{2}$ and $c_{1}, c_{2} \neq 1$, we see that all minors in the corresponding matrix $A_{L}$ of an element $L$ of a such orbit, are non-zero. Therefore, the image of this orbit by the moment map will be the convex hull over all vertices $\Lambda_{i j}, 1 \leq i<j \leq 6$, that is the hypersimplex $\Delta_{5,2}$. If $c_{1}=c_{2} \neq 1$, then $P^{45}\left(A_{L}\right)=0$, while all other minors are non-zero, which implies that the image of the orbit of a such element $L$ will be a four-dimensional polytope, described as the convex hull of the vertices $\Lambda_{i j}$ apart from $\Lambda_{45}$. The proof for the other cases is similar. We take into account the description of orbits given in the proof of Proposition 4.1 and from the set of all vertices remove those corresponding to the minors that are equal to zero.

More geometric description of the polytopes from Proposition 4.2 would be as follows.

Corollary 4.3. The admissible polytopes for $\left(\mathbb{C}^{*}\right)^{5}$ - orbits in the chart $M_{12}$ are:

(1) four-dimensional:

- the hypersimplex $\Delta_{5,2}$;

- any of 9 polytopes with 9 vertices that contains the vertex $\Lambda_{12}$;

- any of 12 polytopes with 8 vertices that contains the vertex $\Lambda_{12}$ and does not contain any of the pairs $\Lambda_{1 i}, \Lambda_{2 j}$ or $\Lambda_{1 i}, \Lambda_{j k}$ or $\Lambda_{2 i}, \Lambda_{j k}$ where $i, j, k \geq 3$ and $i \neq j, k, j \neq k$;

- any of 7 pyramids with 7 vertices whose top vertex is one of the following: $\Lambda_{12}, \Lambda_{13}$, $\Lambda_{14}, \Lambda_{15}, \Lambda_{23}, \Lambda_{24}$ or $\Lambda_{25}$

(2) three-dimensional:

- any of 3 octahedra that contains the pair of vertices $\Lambda_{12}, \Lambda_{34}$ or $\Lambda_{12}, \Lambda_{35}$ or $\Lambda_{12}, \Lambda_{45}$;

- any of 3 pyramids with 5 vertices whose apex is $\Lambda_{12}$;

- any of 6 prisms with vertices $\Lambda_{12}, \Lambda_{1 i}, \Lambda_{1 j}, \Lambda_{2 k}, \Lambda_{i k}, \Lambda_{j k}$ or $\Lambda_{12}, \Lambda_{2 i}, \Lambda_{2 j}, \Lambda_{1 k}, \Lambda_{i k}, \Lambda_{j k}$, where $i, j, k \geq 3, i, j \neq k, i \neq j$;

- any of 12 pyramids with 5 vertices that contains the vertex $\Lambda_{12}$ and whose apex is one of the following: $\Lambda_{13}, \Lambda_{14}, \Lambda_{15}, \Lambda_{23}, \Lambda_{24}$ or $\Lambda_{25}$. 
(3) two-dimensional:

- any of 2 tetrahedra that contain the vertex $\Lambda_{12}$;

- any of 9 triangles that contain the vertex $\Lambda_{12}$;

- any of 6 rectangles that contain the vertex $\Lambda_{12}$;

(4) one-dimensional: any of 6 edges that contains the vertex $\Lambda_{12}$;

(5) zero-dimensional: the vertex $\Lambda_{12}$.

4.3. The admissible polytopes and the strata for $G_{5,2}$. It follows from Lemma 2.24 that any chart contains the same number of admissible polytopes and, in addition, it contains the same number of admissible polytopes of the same type, as given by Proposition 4.3. Moreover, the number of vertices of an admissible polytope $P_{\sigma}$ coincides with the number of charts which contain $W_{\sigma}$. In addition, the number of all charts is equal to the Euler characteristic $\chi\left(G_{5,2}\right)$. Using this we determine the admissible polytopes for $G_{5,2}$ and their numbers.

Proposition 4.4. The admissible polytopes for $G_{5,2} / T^{5}$ are:

(1) four-dimensional:

- hypersimplex $\Delta_{5,2}$;

- 10 polytopes spanned by the 9 vertices;

- 15 polytopes spanned by 8 vertices;

- 10 pyramids spanned by 7 vertices;

(2) three-dimensional:

- 5 octahedra;

- 30 pyramids with 5 vertices;

- 10 prisms with 6 vertices;

- 5 tetrahedra;

(3) two-dimensional:

- 30 triangles;

- 15 squares;

(4) 30 edges;

(5) 10 vertices.

Notations. We set the following notations for some admissible polytopes. The admissible polytope with nine vertices which does not contain the vertex $\Lambda_{i j}$ we denote by $K_{i j}(9), 1 \leq i<$ $j \leq 10$. The admissible polytope with eight vertices which does not contain the vertices $\Lambda_{i j}$ and $\Lambda_{k l}$ we denote by $K_{i j, k l}, 1 \leq i<j \leq 10,1 \leq k<l \leq 10,\{i, j\} \neq\{k, l\}$. The admissible pyramid with seven vertices and with the apex $\Lambda_{i j}$ we denote by $K_{i j}(7), 1 \leq i<j \leq 10$. All polytopes $K_{i j}(9), K_{i j, k l}, K_{i j}(7)$ are four-dimensional. The prisms with six vertices we denote by $P_{i j}, 1 \leq i<j \leq 10$ and it is easy to see they represent base for the pyramids $K_{i j}(7)$. By $O_{i}$, $1 \leq i \leq 5$, we denote the admissible octahedron which does not contain the vertices $A_{i k}$ where $1 \leq k \leq 5, k \neq i$. By $T_{i}, 1 \leq i \leq 5$ we denote the admissible tetrahedron whose vertices are $\Lambda_{i k}, 1 \leq k \leq 5, k \neq i$. The 30 pyramids with five vertices belong to the 5 octahedra and give their polytopal decomposition. The triangles are the facets of the octahedra and tetrahedra, the 15 squares are the diagonal squares for the octahedra, three for each octahedron.

4.4. The combinatorial approach to admissible polytopes. We can approach the description of admissible polytopes in purely combinatorial way. An admissible polytope is the convex hull $\sum_{1 \leq i<j \leq 5} \alpha_{i j} \Lambda_{i j}$, where $0 \leq \alpha_{i j} \leq 1, \sum_{\substack{1 \leq i<j \leq 5 \\ 21}} \alpha_{i j}=1$ and $\Lambda_{i j}$ are the vertices for $\Delta_{5,2}$. The 
numbers $\alpha_{i j}$ can be calculated using the Plücker coordinates as follows:

$$
\alpha_{i j}=\frac{\left|P^{i j}\right|^{2}}{\sum_{1 \leq i<j \leq 5}\left|P^{i j}\right|^{2}}, \quad 1 \leq i<j \leq 5 .
$$

The Plücker coordinates satisfies the relations

$$
P^{i_{1} j_{1}} P^{i_{2} j_{2}}=P^{i_{2} j_{1}} P^{i_{1} j_{2}}-P^{i_{1} i_{2}} P^{j_{1} j_{2}},
$$

which impose the relations on $\alpha_{i j}$. Therefore the admissible polytopes can be described as all possible convex combinations of $\Lambda_{i j}$ where the coefficients of the combinations satisfy the relations imposed from (12). For example,

- if all $\alpha_{i j} \neq 0$ we obtain the admissible polytope $\Delta_{5,2}$;

- if $\alpha_{i_{1} j_{1}}=0$ and $\alpha_{i j} \neq 0$ for $\{i, j\} \neq\left\{i_{1}, j_{1}\right\}$ we obtain the admissible polytope $K_{i_{1} j_{1}}(9)$;

- if $\alpha_{i_{1} j_{1}}=\alpha_{i_{2} j_{2}}=0$ and $\left\{i_{1}, j_{1}\right\} \cap\left\{i_{2}, j_{2}\right\}=\emptyset$ and $\alpha_{i j} \neq 0$ for $\{i, j\} \neq\left\{i_{1}, j_{1}\right\},\left\{i_{2}, j_{2}\right\}$ we obtain the admissible polytope $K_{i_{1} j_{1}, i_{2} j_{2}}$.

- if $\alpha_{i_{1} j_{1}}=\alpha_{i_{1} j_{2}}=0$ and $\alpha_{i_{1} j_{3}} \neq 0$ for $j_{3} \neq j_{1}, j_{2}$ it follows from (12) that $\alpha_{j_{1} j_{2}}=0$. In this way, we obtain the admissible polytope $K_{i_{3} j_{3}}(7)$, where $i_{3}=\{1, \ldots, 5\} \backslash\left\{i_{1}, j_{1}, j_{2}, j_{3}\right\}$.

One can also in a purely combinatorial way verify if some point from $\Delta_{5,2}$ belongs to the interior of a fixed admissible polytope.

Lemma 4.5. A point $x \in \Delta_{5,2}$ belongs to the interior of an admissible polytope $K$ with the vertices $\Lambda_{i_{1} j_{1}}, \ldots, \Lambda_{i_{s} j_{s}}$ if and only of $x=\sum_{l=1}^{s} \alpha_{i_{l} j_{l}} \Lambda_{i_{l} j_{l}}$ where $\sum_{l=1}^{s} \alpha_{i_{1} j_{l}}=1$ and $\alpha_{i_{l} j_{l}}>0$ for all $1 \leq l \leq s$.

The symmetric group $S_{5}$ acts on the set of all admissible polytopes for $G_{5,2}$. We determine here the corresponding stationary subgroups and the fundamental strata.

Lemma 4.6. The stationary subgroups for $S_{5}$-action on the set of all admissible polytopes are isomorphic to:

$$
\begin{gathered}
\Delta_{5,2}: S_{5}, K_{i j}(9): S_{2} \times S_{3}, K_{i j, k l}: S_{2} \times S_{2} \times S_{2} ; K_{i j}(7): S_{2} \times S_{3}, P_{i j}: S_{2} \times S_{3}, \\
O_{i}: S_{4}, T_{i}: S_{4}, \text { five-vertex pyramids }: S_{2} \times S_{2}, \\
\text { squares }: S_{2} \times S_{2} \times S_{2}, \text { triangles }: S_{3} \text { or } S_{2} \times S_{3} \\
\text { edges }: S_{2} \times S_{2} \text {, vertices : } S_{2} \times S_{3} .
\end{gathered}
$$

Proof. We demonstrate the proof for the admissible triangles. The vertices of an admissible triangle are $\left\{\Lambda_{i j}, \Lambda_{i k}, \Lambda_{i l}\right\}$ or $\left\{\Lambda_{i j}, \Lambda_{i k}, \Lambda_{j k}\right\}$, where $i, j, k, l$ are pairwise distinct. The stationary subgroup for the triangles of the first form is $S_{3}$ and for the triangles of the second form it is $S_{2} \times S_{3}$.

Lemma 4.7. The fundamental polytopes for $G_{5,2}$ for admissible polytopes which:

- belong to the interior of $\Delta_{5,2}$ are: $\Delta_{5,2}$, one $K_{i j}(9)$, one $K_{i j, k l}$, one $K_{i j}(7)$ and one $P_{i j}$;

- belong to the boundary of $\Delta_{5,2}$ are: one $O_{i}$, one $T_{i}$, one square, two triangles, one edge and one vertex.

Proof. The stationary subgroup for $K_{i j}(9)$ is isomorphic to $S_{2} \times S_{3}$ which has the order 12 . Since the order of $S_{5}$ is 120 we obtain that the $S_{5}$-orbit of $K_{i j}(9)$ has 10 elements. This coincides with the number of the admissible polytopes with 9 vertices. The proof for the other admissible polytopes, apart from triangles and edges, is analogous. The orbit of a triangle, whose stationary subgroup 
is $S_{3}$ has 20 elements, while the orbit of a triangle, whose stationary subgroup is $S_{2} \times S_{3}$ has 10 elements. Altogether this gives 30 triangles.

Corollary 4.8. The number of the strata for $T^{5}$-action on $G_{5,2}$ is $125+46=171$. The number of the fundamental strata is 13 , where $q_{3}=q_{4}=q_{6}=2$ and $q_{p}=1$ for $p \neq 3,4,6$.

4.5. The closure of $\left(\mathbb{C}^{*}\right)^{5}$-orbits. The closure of a $\left(\mathbb{C}^{*}\right)^{5}$-orbit in $G_{5,2}$ is a toric variety. Following the arguments from [7] we provide the description of these toric varieties.

Theorem 4.9. The closure of an orbit for $\left(\mathbb{C}^{*}\right)^{5}$-action on $G_{5,2}$ is a toric variety:

(1) of dimension eight (complex dimension 4) and with:

- 10 singular points if its admissible polytope is $\Delta_{5,2}$;

- 9 singular points if its admissible polytope is $K_{i j}(9), 1 \leq i<j \leq 5$;

- 4 singular points if its admissible polytope is $K_{i j, k l}, 1 \leq i, j, k, l \leq 5, i \neq j \neq k \neq l$;

- 1 singular point if its admissible polytope is $K_{i j}(7), 1 \leq i<j \leq 5$;

(2) of dimension six (complex dimension 3):

- with 6 singular points if its admissible polytope is an octahedra $O_{i}, 1 \leq i \leq 5$;

- with 1 singular point if its admissible polytope is a pyramid over square;

- $\mathbb{C} P^{2} \times \mathbb{C} P^{1}$ if its admissible polytope is a prism $P_{i j}, 1 \leq i<l \leq 5$;;

- $\mathbb{C} P^{3}$ if its admissible polytope is a tetrahedra $T_{i}, 1 \leq i \leq 5$;

(3) of dimension four:

- $\mathbb{C} P^{2}$ if its admissible polytope is a triangle;

- $\mathbb{C} P^{1} \times \mathbb{C} P^{1}$ if its admissible polytope is a rectangle;

(4) of dimension two, i. e. $\mathbb{C} P^{1}$.

(5) of dimension zero vertices, $i$. e. fixed points.

All singular points of these toric varieties map, by the moment map, to the vertices of $\Delta_{5,2}$.

As for the regular point of the moment map, Corollary 3.10 implies:

Corollary 4.10. All points of the eight-dimensional orbits for $\left(\mathbb{C}^{*}\right)^{5}$-action on $G_{5,2}$ are the regular points for the moment map $\mu: G_{5,2} \rightarrow \Delta_{5,2}$.

The only $l$ - dimensional admissible polytopes, $l \leq 3$, which intersect $\stackrel{\circ}{\Delta}_{5,2}$ are the prisms $P_{i j}$. More precisley, $\stackrel{\circ}{P}_{i j} \subset \stackrel{\circ}{\Delta}_{5,2}$. Thus, Theorem 3.11 implies:

Corollary 4.11. The set of singular values in $\stackrel{\circ}{\Delta}_{5,2}$ for the moment map $\mu$ is given by $\cup_{1 \leq i<j \leq 5} \stackrel{\circ}{P}_{i j}$. The set of singular points in $G_{5,2}$, which are mapped by the moment map to $\Delta_{5,2}$, is given by the principal orbits of the toric varieties $W_{i j} \cong \mathbb{C} P^{2} \times \mathbb{C} P^{1}$. The toric varieties $W_{i j}$ are mapped, by the moment map, to $P_{i j}$, where $1 \leq i<j \leq 5$.

By Proposition 3.14, the toric varieties $W_{i j} \cong \mathbb{C} P^{2} \times \mathbb{C} P^{1} \subset G_{5,2}$ have a nice geometric description:

Corollary 4.12. The ten toric varieties $W_{i j}, 1 \leq i<j \leq 5$ correspond to the ten decomposition $\mathbb{C}^{5}=\mathbb{C}^{3} \times \mathbb{C}^{2}$. More precisely, $W_{i j}$ consist of two-dimensional subspaces $L \subset \mathbb{C}^{5}$ such that $L=$ $\mathcal{L}\left(L_{1}, L_{2}\right)$, where $L_{1}, L_{2}$ are the lines in $\mathbb{C}^{3}, \mathbb{C}^{2}$ respectively, for the corresponding decomposition of $\mathbb{C}^{5}=\mathbb{C}^{3} \times \mathbb{C}^{2}$. 
4.6. The strata in the chart $M_{12}$, their spaces of parameters and orbit spaces. We analyze now the spaces of parameters of the strata as well as the $T^{5}$ - orbit spaces of the strata. Since the action of $S_{5}$ on $G_{5,2}$ permutes the charts, the strata and, consequently, the admissible polytopes and the spaces of parameters, it is enough to consider these objects just in an one chart.

From Proposition 4.1 and Proposition 4.2 it directly follows:

Corollary 4.13. Any stratum $W_{\sigma}$ for which $P_{\sigma} \neq \Delta_{5,2}, K_{i j}(9), O_{l}$ consists of one orbit. Consequently, $F_{\sigma}$ is a point.

The main stratum $W$ consists of the points whose all Plücker coordinates are non-zero. The main stratum belongs to all chart for $G_{5,2}$, so Proposition 4.1 implies:

Corollary 4.14. The main stratum is, in a fixed chart, given by the following system of equations:

$$
c_{1}^{\prime} z_{1} z_{5}=c_{1} z_{2} z_{4}, \quad c_{2}^{\prime} z_{1} z_{6}=c_{2} z_{3} z_{4}, \quad c_{3}^{\prime} z_{2} z_{6}=c_{3} z_{3} z_{5},
$$

where

$$
c_{1} c_{2}^{\prime} c_{3}=c_{1}^{\prime} c_{2} c_{3}^{\prime} \text { and } c_{i}, c_{i}^{\prime} \neq 0, c_{i} \neq c_{i}^{\prime}, c_{i}, c_{i}^{\prime} \in \mathbb{C} \text {. }
$$

Corollary 4.15. The space of parameters $F$ of the main stratum $W$ is homeomorphic to

$$
\left\{\left(\left(c_{1}: c_{1}^{\prime}\right),\left(c_{2}: c_{2}^{\prime}\right),\left(c_{3}: c_{3}^{\prime}\right)\right) \in \mathbb{C} P^{1} \times \mathbb{C} P^{1} \times \mathbb{C} P^{1}, \quad c_{1} c_{2}^{\prime} c_{3}=c_{1}^{\prime} c_{2} c_{3}^{\prime}, \quad c_{i}, c_{i}^{\prime} \neq 0, \quad c_{i} \neq c_{i}^{\prime}\right\} .
$$

The other non-orbit strata are, in the chart $M_{12}$, given by the family of surfaces (1), (2) and (3) from Proposition 4.1, Therefore we have:

Corollary 4.16. The space of parameters for a non-orbit stratum, which is different from the main stratum, that is for a stratum whose admissible polytope is $K_{i j}(9)$ or $O_{i}$, is homeomorphic to $\mathbb{C} P_{A}^{1}=\mathbb{C} P^{1} \backslash A$, where $A=\{(0: 1),(1: 0),(1: 1)\}$.

From Theorem 3.6 it follows:

Corollary 4.17. The orbit space $W_{\sigma} / T^{5}$ of a stratum $W_{\sigma}$ whose admissible polytope is:

(1) $\Delta_{5,2}$ is homeomorphic to $\stackrel{\circ}{\Delta}_{5,2} \times F$;

(2) $K_{i j}(9)$ is homeomorphic to $\stackrel{\circ}{K}_{i j}(9) \times \mathbb{C} P_{A}^{1}$;

(3) $O_{i}$ is homeomorphic to $\stackrel{\circ}{O}_{i} \times \mathbb{C} P_{A}^{1}$.

Corollary 4.18. The orbit space for $W_{\sigma} / T^{5}$ of a stratum $W_{\sigma}$ whose admissible polytope $P_{\sigma}$ is different from $\Delta_{5,2}, K_{i j}(9)$ and $O_{i}$ is homeomorphic to $\stackrel{\circ}{P_{\sigma}}$.

Remark 4.19. Let us fix a chart $M_{i j}$ and let $W_{\sigma} \subset M_{i j}$ be a stratum such that $F_{\sigma}$ is not a point. Then, as Proposition 4.1 shows, $W_{\sigma}$ is defined by the equations whose coefficients belong to the space of parameters $F_{\sigma}$. We denote by $F_{\sigma, i j}$ a coordinate record of the space of parameters $F_{\sigma}$ in the chart $M_{i j}$. By $f_{\sigma, i j}: F_{\sigma, i j} \rightarrow F_{\sigma}$ we denote the induced canonical homeomorphism. We want to emphasize that if $F_{\sigma}$ is a point then one can not assign to the point $F_{\sigma}$ the parameter coordinates in the chart $M_{i j}$, since in this case the stratum $W_{\sigma}$ consists of one $\left(\mathbb{C}^{*}\right)^{5}$-orbit and by Proposition 4.1 no equations are imposed on the points of $W_{\sigma}$.

Summing up all we obtain: 
Proposition 4.20. The strata for $\left(\mathbb{C}^{*}\right)^{5}$-action on $G_{5,2} / T^{5}$, their number in each dimension and corresponding polytopes are given as follows:

(15)

\begin{tabular}{|c|c|c|c|c|c|c|c|c|c|c|c|}
\hline 12 & 10 & 8 & 8 & 8 & 6 & 6 & 6 & 4 & 4 & 2 & 0 \\
\hline 1 & 10 & 15 & 10 & 5 & 10 & 30 & 5 & 15 & 30 & 30 & 10 \\
\hline$\Delta_{5,2}$ & $\mathbf{K}_{\mathbf{i j}}(\mathbf{9})$ & $K_{i j, k l}(8)$ & $K_{i j}(7)$ & $\mathrm{O}_{\mathbf{i}}(6)$ & $P_{i j}(6)$ & $P(6)$ & $T_{i}(4)$ & $P(4)$ & $P(3)$ & $P(2)$ & Ver \\
\hline
\end{tabular}

Apart from the bolded strata, all other strata consist of one orbit.

4.7. The facets of the admissible polytopes. We describe here the facets of the four-dimensional admissible polytopes. Using the description of the main stratum in the chart $M_{12}$ we obtain:

Proposition 4.21. The facets of the hypersimplex $\Delta_{5,2}$ are the octahedra $O_{i}$ and the tetrahedra $T_{i}$, where $1 \leq i \leq 5$.

We describe first the facets of the admissible polytopes $K_{i j}(9)$. For the clearness of the exposition we demonstrate it for the polytope $K_{13}(9)$. We denote by $P_{i}^{k l}, k, l \neq i$, the four sided prism spanned by the vertices of the octahedron $O_{i}$ excluding the vertex $\Lambda_{k l}$.

Proposition 4.22. The boundary of $K_{13}(9)$ consists of the octahedra $O_{1}$ and $O_{3}$, the tetrahedra $T_{2}, T_{4}$ and $T_{5}$, the pyramids $P_{2}^{13}, P_{4}^{13}$ and $P_{5}^{13}$ and the prism $P_{13}$ with the based triangles spanned by $\left\{\Lambda_{12}, \Lambda_{14}, \Lambda_{15}\right\}$ and $\left\{\Lambda_{23}, \Lambda_{34}, \Lambda_{35}\right\}$.

Proof. The stratum $W_{\sigma}, \sigma=\{I \subset\{1, \ldots, 5\},|I|=2\} \backslash\{1,3\}$, which maps to $K_{13}(9)$ is, in the chart $M_{12}$, given by the following system of equations $c^{\prime} z_{2} z_{6}=c z_{3} z_{5}$ and $z_{4}=0$, where $\left(c: c^{\prime}\right) \in \mathbb{C} P_{A}^{1}$. The boundary of any $\left(\mathbb{C}^{*}\right)^{5}$-orbit from $W_{\sigma}$ consists of the $\left(\mathbb{C}^{*}\right)^{5}$-orbits of the smaller dimensions and there is a bijection between these orbits and the faces of $K_{13}(9)$. The orbits of complex dimension 3 , belonging to the boundary of an orbit from $W_{\sigma}$ are given by the following conditions: $z_{1}=0$ ( its polytope is $O_{3}$ ), $z_{2}=z_{3}=0$ (its polytope is $P_{13}$ ), $z_{2}=z_{5}=0$ ( its polytope is $P_{4}^{13}$ ), $z_{3}=z_{6}=0$ (its polytope is the pyramid $P_{5}^{13}\left(, z_{5}=z_{6}=0\right.$ ( its polytope is $T_{2}$ ). The stationary subgroup for $K_{13}(9)$ regarded to $S_{5}$-action is $S_{13}=S_{2}\{1,3\} \times S_{3}\{2,4,5\}$. By the action of $S_{13}$ on those facets of $K_{13}(9)$, which belong to the chart $M_{12}$ we obtain all facets for $K_{13}(9)$. In this way in addition we obtain $O_{1}, P_{2}^{13}, T_{4}$ and $T_{5}$ to be the facets for $K_{13}(9)$.

More generally:

Proposition 4.23. The boundary of $K_{i j}(9)$ consists of the octahedra $O_{i}$ and $O_{j}$, the tetrahedra $T_{k}, T_{l}, T_{m}$, the pyramids $P_{k}^{i j}, P_{l}^{i j}$ and $P_{m}^{i j}$ and the prism $P_{i j}$ with the based triangles spanned by $\left\{\Lambda_{i k}, \Lambda_{i l}, \Lambda_{i m}\right\}$ and $\left\{\Lambda_{j k}, \Lambda_{j l}, \Lambda_{j m}\right\}$, where $k, l, m \neq i, j$.

In an analogous way we describe the facets for the polytopes $K_{i j, k l}$ and $K_{i j}(7)$.

Proposition 4.24. The boundary of the polytope $K_{i j, k l}$ consists of two prisms $P_{i j}$ and $P_{k l}$ with the based triangles spanned by $\left\{\Lambda_{i s}, \Lambda_{i p}, \Lambda_{i q}\right\},\left\{\Lambda_{j s}, \Lambda_{j p}, \Lambda_{j q}\right\}$ and $\left\{\Lambda_{k r}, \Lambda_{k u}, \Lambda_{k v}\right\},\left\{\Lambda_{l r}, \Lambda_{l u}, \Lambda_{l v}\right\}$, the tetrahedron $T_{m}$ and the pyramids $P_{i}^{k l}, P_{j}^{k l}, P_{k}^{i j}, P_{l}^{i j}$, where $\{s, p, q\}=\{1,2,3,4,5\} \backslash\{i, j\}$, $\{r, u, v\}=\{1,2,3,4,5\} \backslash\{k, l\}$ and $m \neq i, j, k, l$ while $i, j \neq k, l$.

Remark 4.25. An admissible polytope $K_{i j, k l}$ has eight vertices, among them four vertices are simple, while the other four vertices have five edges.

Proposition 4.26. The boundary of the polytope $K_{i j}(7)$ consists of the prism $P_{i j}$ with the based triangles spanned by $\left\{\Lambda_{i p}, \Lambda_{i q}, \Lambda_{i r}\right\},\left\{\Lambda_{j p}, \Lambda_{j q}, \Lambda_{j r}\right\}$, the tetrahedra $T_{i}, T_{j}$ and the pyramids $P_{p}^{q r}$, $P_{q}^{p r}, P_{r}^{p q}$, where $\{p, q, r\}=\{1,2,3,4,5\} \backslash\{i, j\}$. 
Corollary 4.27. The prism $P_{i j}$ with the based triangles spanned by $\left\{\Lambda_{i p}, \Lambda_{i q}, \Lambda_{i r}\right\}$ and $\left\{\Lambda_{j p}, \Lambda_{j q}, \Lambda_{j r}\right\}$ is the common interior facet for $K_{i j}(9)$, polytopes $K_{i j, k l}$ and pyramid $K_{i j}(7)$. It is the only facet for $K_{i j}(9)$ and $K_{i j}(7)$ that belongs to the interior of $\stackrel{\circ}{\Delta}_{5,2}$.

Note that the polytopes $K_{i l, k l}, K_{i j, p q}, K_{i j, r s}, K_{k l}(7), K_{p q}(7), K_{r s}(7)$ are sub-polytopes of $K_{i j}(9)$ and that any of the pairs $\left(K_{i j, k l}, K_{k l}(7)\right),\left(K_{i j, p q}, K_{p q}(7)\right),\left(K_{i j, r s}, K_{r s}(7)\right)$ gives the polytopal decomposition of $K_{i j}(9)$. On the other hand, the pairs $\left(K_{i j}(9), K_{i j}(7)\right)$ give the polytopal decomposition of $\Delta_{5,2}$, where $K_{i j}(9)$ and $K_{i j}(7)$ are glued together along the prism $P_{i j}$, which is their common interior facet.

\section{THE IDEA OF THE UNIVERSAL SPACE OF PARAMETERS}

The main stratum is an everywhere dense set in $G_{5,2}$ meaning that any other stratum is in the boundary of the main stratum. Using that fact our goal is to prove the following theorem.

Theorem 5.1. There exists a topological space $\tilde{\mathcal{F}}$ such that:

(1) for any chart $M_{i j}$ there is a map $H_{i j}: \mathcal{A} \rightarrow \tilde{\mathcal{F}}, \sigma \rightarrow \tilde{F}_{\sigma, i j}$, where $\tilde{F}_{\sigma, i j} \subset \tilde{\mathcal{F}}$ is defined by: $c \in \tilde{F}_{\sigma, i j}$ if and only if there exists a sequence $\left(x_{n}, c_{n}\right) \subset \stackrel{\circ}{\Delta}_{5,2} \times F_{i j}$ such that $c_{n}$ converges to $c$ and $h^{-1}\left(x_{n}, f_{i j}\left(c_{n}\right)\right)$ converges to a point from $W_{\sigma} / T^{5}$.

(2) $\tilde{F}_{\sigma, i j}$ is homeomorphic to $\tilde{F}_{\sigma, k l}$ for any charts $M_{i j}, M_{k l}$.

Here $\mathcal{A}$ is the set of all admissible sets, $h: W / T^{5} \rightarrow \Delta_{5,2}^{\circ} \times F$ is the canonical trivialization of the main stratum and $f_{i j}: F_{i j} \rightarrow F$ is the canonical homeomorphism for the chart $M_{i j}$.

Let us explain the meaning of this theorem a little more. The orbit space $G_{5,2} / T^{5}$ is a compactification of $W / T^{5}$ and the boundary of $W / T^{5}$ in $G_{5,2} / T^{5}$ is given by $\cup_{W_{\sigma} \neq W} W_{\sigma} / T^{5}$. Theorem 3.6 states that for any $\sigma \in \mathcal{A}, W_{\sigma} / T^{n}$ is homeomorphic to $\stackrel{\circ}{P}_{\sigma} \times F_{\sigma, i j}$. Theorem 7.3 claims that there exists a corresponding compactification for $\stackrel{\circ}{\Delta}_{5,2} \times F_{i j}$ which is given by the subspaces $\tilde{F}_{\sigma, i j} \subset \tilde{\mathcal{F}}$, that is a compactification for $\stackrel{\circ}{\Delta}_{5,2} \times F_{i j}$ of the form $\cup_{\sigma} \stackrel{\circ}{P}_{\sigma} \times \tilde{F}_{\sigma, i j}$.

Definition 5.2. The space $\tilde{\mathcal{F}}$ is called the universal space of parameters for $T^{5}$-action on $G_{5,2}$.

Definition 5.3. The spaces $\tilde{F}_{\sigma, i j}$ are said to be the virtual spaces of parameters for the strata $W_{\sigma}$ in the chart $M_{i j}$.

Note that Theorem 7.3 directly implies that $F_{i j} \subset \tilde{\mathcal{F}}$ for any chart $M_{i j}$. We know that $F_{i j} \subset$ $\mathbb{C} P^{1} \times \mathbb{C} P^{1} \times \mathbb{C} P^{1}$ and we will prove that the closure $\bar{F}_{i j} \subset \mathbb{C} P^{1} \times \mathbb{C} P^{1} \times \mathbb{C} P^{1}$ has to belong to $\tilde{\mathcal{F}}$ as well. But, as the consideration that follows will show this closure is not enough, the universal space of parameters turns out to be wider.

We proceed with the proof of Theorem 7.3 though few steps. First, we describe the subsets $\bar{F}_{\sigma, 12} \subset \bar{F}_{12}$ for the strata $W_{\sigma}$, which belong to the chart $M_{12}$. The subsets $\bar{F}_{\sigma, 12}$ are obtained using the fact that the main stratum is a dense set in this chart. This description will show that in finding $\tilde{\mathcal{F}}$ we should start with $\bar{F} \subset \mathbb{C} P^{1} \times \mathbb{C} P^{1} \times \mathbb{C} P^{1}$. Then we describe the automorphisms of $F$ given by the transition homeomorphisms between $F_{i j}$ and $F_{k l}$ in different charts $M_{i j}, M_{k l}$. Finally we find the compactification $\tilde{\mathcal{F}}$ for $F$ such that any of these automorphism extends to an automorphism of $\tilde{\mathcal{F}}$. This compactification will satisfy the conditions of Theorem 7.3 , 


\section{The SPACES $\bar{F}_{\sigma, 12}$ FOR THE STRATA $W_{\sigma}$ IN THE CHART $M_{12}$}

We describe the spaces $\bar{F}_{\sigma, 12} \subset \bar{F}_{12}$ for the strata which belong to the chart $M_{12}$.

Proposition 6.1. The spaces $\bar{F}_{i j, 12}$ for the strata whose admissible polytopes are $K_{i j}(9)$ and which belong to the chart $M_{12}$, are $\bar{F}_{i j, 12} \subset \bar{F}_{12} \subset \mathbb{C} P^{1} \times \mathbb{C} P^{1} \times \mathbb{C} P^{1}$ given as follows:

(1) $\bar{F}_{23,12} \rightarrow\left((0: 1),(0: 1),\left(c: c^{\prime}\right)\right)$,

(2) $\bar{F}_{24,12} \rightarrow\left((1: 0),\left(c: c^{\prime}\right),(0: 1)\right)$,

(3) $\bar{F}_{25,12} \rightarrow\left(\left(c: c^{\prime}\right),(1: 0),(1: 0)\right)$,

(4) $\bar{F}_{13,12} \rightarrow\left((1: 0),(1: 0),\left(c: c^{\prime}\right)\right)$,

(5) $\bar{F}_{14,12} \rightarrow\left((0: 1),\left(c: c^{\prime}\right),(1: 0)\right)$,

(6) $\bar{F}_{15,12} \rightarrow\left(\left(c: c^{\prime}\right),(0: 1),(0: 1)\right)$,

(7) $\bar{F}_{34,12} \rightarrow\left((1: 1),\left(c: c^{\prime}\right),\left(c: c^{\prime}\right)\right)$,

(8) $\bar{F}_{35,12} \rightarrow\left(\left(c: c^{\prime}\right),(1: 1),\left(c^{\prime}: c\right)\right)$,

(9) $\bar{F}_{45,12} \rightarrow\left(\left(c: c^{\prime}\right),\left(c: c^{\prime}\right),(1: 1)\right)$,

where $\left(c: c^{\prime}\right) \in \mathbb{C} P_{A}^{1}$.

Proof. The strata (1) - (9) belong to the chart $M_{12}$ and the given list of their spaces of parameters follows from the description of these strata given by Proposition 4.1. The stratum (1) is, in the chart $M_{12}$, given by the condition $z_{1}=0, z_{i} \neq 0, i \neq 1$ and $\frac{z_{2} z_{6}}{z_{3} z_{5}} \neq 1$. So, if a sequence of points $\left(z_{1}^{n}, \ldots, z_{6}^{n}\right)$ from the main stratum converges to a given point from the stratum (1), it follows that $z_{1}^{n} \rightarrow 0$, while $z_{i}^{n} \rightarrow z_{i} \neq 0$. Thus, $c_{1}^{n}=\frac{z_{1}^{n} z_{5}^{n}}{z_{2}^{n} z_{4}^{n}} \rightarrow 0, c_{2}^{n}=\frac{z_{1}^{n} z_{6}^{n}}{z_{3}^{n} z_{4}^{n}} \rightarrow 0$, while $\frac{c_{1}^{n}\left(c_{1}^{n}-1\right)}{c_{2}^{n}\left(c_{2}^{n}-1\right)} \rightarrow c$, where $c=\frac{z_{2} z_{6}}{z_{3} z_{5}}$. Therefore the orbits from the stratum (1) are continuously parametrized by triples $(0,0, c)$, where $c \in \mathbb{C}-\{0,1\}$. In the same way, we deduce the parametrization for the orbits from the strata $(2)-(9)$.

Proposition 6.2. The spaces $\bar{F}_{(i j, k l), 12}$ for the strata whose admissible polytopes are $K_{i j, k l}$ and which belong to the chart $M_{12}$, are $\bar{F}_{(i j, k l), 12} \subset \bar{F}_{12} \subset \mathbb{C} P^{1} \times \mathbb{C} P^{1} \times \mathbb{C} P^{1}$ given as follows:

- $\bar{F}_{(14,23), 12} \rightarrow((0: 1),(0: 1),(1: 0)), \bar{F}_{(13,24), 12} \rightarrow((1: 0),(1: 0),(0: 1))$,

- $\bar{F}_{(15,24), 12} \rightarrow((1: 0),(0: 1),(0: 1)), \bar{F}_{(23,45), 12} \rightarrow((0: 1),(0: 1),(1: 1))$,

- $\bar{F}_{(24,35), 12} \rightarrow((1: 0),(1: 1),(0: 1)), \bar{F}_{(25,34), 12} \rightarrow((1: 1),(1: 0),(1: 0))$,

- $\bar{F}_{(15,23), 12} \rightarrow((0: 1),(0: 1),(0: 1)), \bar{F}_{(13,25), 12} \rightarrow((1: 0),(1: 0),(1: 0))$,

- $\bar{F}_{(14,25), 12} \rightarrow((0: 1),(1: 0),(1: 0)), \quad \bar{F}_{(13,45), 12} \rightarrow((1: 0),(1: 0),(1: 1))$;

- $\bar{F}_{(14,35), 12} \rightarrow((0: 1),(1: 1),(1: 0)), \bar{F}_{(15,34), 12} \rightarrow((1: 1),(0: 1),(0: 1))$.

Proposition 6.3. The spaces $\bar{F}_{i j, 12}(7)$ for the strata whose admissible polytopes are $K_{i j}(7)$ and which belong to the chart $M_{12}$, are $\bar{F}_{i j, 12}(7) \subset \bar{F}_{12} \subset \mathbb{C} P^{1} \times \mathbb{C} P^{1} \times \mathbb{C} P^{1}$ given as follows:

- $\bar{F}_{23,12}(7) \rightarrow(0,1) \times(0: 1) \times \mathbb{C} P^{1}, \quad \bar{F}_{24,12}(7) \rightarrow(1: 0) \times \mathbb{C} P^{1} \times(0: 1)$,

- $\bar{F}_{25,12}(7) \rightarrow \mathbb{C} P^{1} \times(1: 0) \times(1: 0), \quad \bar{F}_{13,12}(7) \rightarrow(1: 0) \times(1: 0) \times \mathbb{C} P^{1}$,

- $\bar{F}_{14,12}(7) \rightarrow(0: 1) \times \mathbb{C} P^{1} \times(1: 0), \quad \bar{F}_{15,12}(7) \rightarrow \mathbb{C} P^{1} \times(0: 1) \times(0: 1)$,

- $\bar{F}_{12,12}(7) \rightarrow((1: 1),(1: 1),(1: 1))$.

Proposition 6.4. The spaces $\bar{F}_{i, 12}$ for the strata whose admissible polytopes are $O_{i}$ and which belong to the chart $M_{12}$, are the subsets $\bar{F}_{i, 12} \subset \bar{F}_{12} \subset \mathbb{C} P^{1} \times \mathbb{C} P^{1} \times \mathbb{C} P^{1}$ given as follows:

- $\bar{F}_{3,12} \rightarrow \bar{F}_{12} \cap\left(\mathbb{C} P^{1} \times \mathbb{C} P^{1} \times \mathbb{C} P_{A}^{1}\right)$,

- $\bar{F}_{4,12} \rightarrow \bar{F}_{12} \cap\left(\mathbb{C} P^{1} \times \mathbb{C} P_{A}^{1} \times \mathbb{C} P^{1}\right)$, 


$$
\text { - } \bar{F}_{5,12} \rightarrow \bar{F}_{12} \cap\left(\mathbb{C} P_{A}^{1} \times \mathbb{C} P^{1} \times \mathbb{C} P^{1}\right) \text {. }
$$

Proposition 6.5. The spaces $\bar{F}_{i j, 12}(6)$ for the strata whose admissible polytopes are $P_{i j}$ and which belong to the chart $M_{12}$, are $\bar{F}_{i j, 12}(6) \subset \bar{F}_{12} \subset \mathbb{C} P^{1} \times \mathbb{C} P^{1} \times \mathbb{C} P^{1}$ given as as follows:

- $\bar{F}_{23,12}(6) \rightarrow(0,1) \times(0: 1) \times \mathbb{C} P^{1}, \quad \bar{F}_{24,12}(6) \rightarrow(1: 0) \times \mathbb{C} P^{1} \times(0: 1)$,

- $\bar{F}_{25,12}(6) \rightarrow \mathbb{C} P^{1} \times(1: 0) \times(1: 0), \bar{F}_{13,12}(6) \rightarrow(1: 0) \times(1: 0) \times \mathbb{C} P^{1}$,

- $\bar{F}_{14,12}(6) \rightarrow(0: 1) \times \mathbb{C} P^{1} \times(1: 0), \bar{F}_{15,12}(6) \rightarrow \mathbb{C} P^{1} \times(0: 1) \times(0: 1)$,

Remark 6.6. It holds $\bar{F}_{3,12}=\bar{F}_{12} \cap\left(\mathbb{C} P^{1} \times \mathbb{C} P^{1} \times \mathbb{C} P_{A}^{1}\right)=F_{12} \cup \bar{F}_{13,12} \cup \bar{F}_{23,12} \cup \bar{F}_{34,12} \cup \bar{F}_{35,12}$ and this corresponds to the fact that $O_{3}$ is a facet for each of $\Delta_{5,2}, K_{13}(9), K_{23}(9), K_{34}(9)$ and $K_{35}(9)$. In other words orbits from the stratum whose admissible polytope is $\mathrm{O}_{3}$ are in the boundary of the orbits of the strata with the admissible polytopes $\Delta_{5,2}, K_{13}(9), K_{23}(9), K_{34}(9)$ and $K_{35}(9)$. Then using the action of the symmetric group $S_{5}$ we immediately obtain the description for $\bar{F}_{4,12}$ and $\bar{F}_{5,12}$.

In an analogous way we can describe the spaces of parameters $\bar{F}_{\sigma, 12} \subset \bar{F}_{12}$ for any stratum $W_{\sigma} \subset M_{12}$. We do not find necessary to list all of them here since the admissible polytopes for all strata, which are not listed in the previous propositions, are faces of $\Delta_{5,2}$ and their spaces of parameters are homeomorphic to a point.

Remark 6.7. The previous propositions suggest that in order to find an universal space of parameters we should start with the closure of the space of parameters $F$ in $\mathbb{C} P^{1} \times \mathbb{C} P^{1} \times \mathbb{C} P^{1}$.

Remark 6.8. The stratum whose admissible polytope is $K_{13}(7)$ is, in the chart $M_{12}$, parametrized by $((1: 1),(1: 1),(1: 1)) \in \bar{F}_{12}$ according to Proposition 6.3 . This stratum belongs to the chart $M_{13}$ as well and, using the same argument as above, it can be seen that, in that chart $M_{13}$, this stratum is parametrized by $(1: 0) \times(1: 0) \times \mathbb{C} P^{1} \subset \bar{F}_{13}$. Since the virtual spaces of parameters $\tilde{F}_{\sigma, i j}$ of a stratum $W_{\sigma}$ in different charts should be homeomorphic, this shows that the closure $\bar{F}$ of $F$ in $\mathbb{C} P^{1} \times \mathbb{C} P^{1} \times \mathbb{C} P^{1}$ can not be taken as an universal space of parameters.

\section{THE UNIVERSAL SPACE OF PARAMETERS $\tilde{\mathcal{F}}$}

7.1. The closure $\mathcal{F}$ of $F$ in $\mathbb{C} P^{1} \times \mathbb{C} P^{1} \times \mathbb{C} P^{1}$. The closure of the space $F_{k l} \subset \mathbb{C} P^{1} \times \mathbb{C} P^{1} \times \mathbb{C} P^{1}$, which represent the space of parameters of the main stratum in the chart $M_{k l}$, should not depend on the chart $M_{k l}$, since all our constructions are invariant under the action of the symmetric group $S_{5}$. The closure of $F_{k l}$ in $\mathbb{C} P^{1} \times \mathbb{C} P^{1} \times \mathbb{C} P^{1}$ is:

$$
\bar{F}_{k l}=\left\{\left(\left(c_{1, k l}: c_{1, k l}^{\prime}\right),\left(c_{2, k l}: c_{2, k l}^{\prime}\right),\left(c_{3, k l}: c_{3, k l}^{\prime}\right)\right) \mid c_{1, k l} c_{2, k l}^{\prime} c_{3, k l}=c_{1, k l}^{\prime} c_{2, k l} c_{3, k l}^{\prime}\right\} .
$$

Because of our further purposes we describe this closure in more detail. We obtain the points from the boundary of $F_{k l}$ if we put in the cubic equation $c_{1, k l} c_{2, k l}^{\prime} c_{3, k l}=c_{1, k l}^{\prime} c_{2, k l} c_{3, k l}^{\prime}$ that some $c_{i, k l}=0$, $c_{i, k l}^{\prime}=0$ or $c_{i, k l}=c_{i, k l}^{\prime}$, but keeping that $c_{i, k l}, c_{i, k l}^{\prime}$ may not be both zero, where $1 \leq i \leq 3$. The explicit description of all such points is:

Lemma 7.1. The boundary of $F_{k l} \subset \mathbb{C} P^{1} \times \mathbb{C} P^{1} \times \mathbb{C} P^{1}$ consists of the following sets:

$$
\begin{gathered}
\bar{F}_{k l}^{12}=(0: 1) \times(0: 1) \times \mathbb{C} P^{1}, \quad \bar{F}_{k l}^{13^{\prime}}=(0: 1) \times \mathbb{C} P^{1} \times(1: 0), \\
\bar{F}_{k l}^{1^{\prime} 2^{\prime}}=(1: 0) \times(1: 0) \times \mathbb{C} P^{1}, \quad \bar{F}_{k l}^{1^{\prime} 3}=(1: 0) \times \mathbb{C} P^{1} \times(0: 1), \\
\bar{F}_{k l}^{23}=\mathbb{C} P^{1} \times(0: 1) \times(0: 1), \bar{F}_{k l}^{2^{\prime} 3^{\prime}}=\mathbb{C} P^{1} \times(1: 0) \times(1: 0),
\end{gathered}
$$




$$
\begin{gathered}
\bar{F}_{k l}^{11^{\prime}}=\left\{\left((1: 1),\left(c_{2, k l}: c_{2, k l}^{\prime}\right),\left(c_{2, k l}: c_{2, k l}^{\prime}\right)\right)\right\}, \bar{F}_{k l}^{22^{\prime}}=\left\{\left(\left(c_{1, k l}: c_{1, k l}^{\prime}\right),(1: 1),\left(c_{1, k l}^{\prime}: c_{1, k l}^{\prime}\right)\right)\right\}, \\
\bar{F}_{k l}^{33^{\prime}}=\left\{\left(\left(c_{1, k l}: c_{1, k l}^{\prime}\right),\left(c_{1, k l}: c_{1, k l}^{\prime}\right),(1: 1)\right)\right\} .
\end{gathered}
$$

Proof. We consider the cubic equation in (19) and directly deduce the following: $c_{1, k l}=c_{2, k l}=0$ gives $\bar{F}_{k l}^{12}, c_{1, k l}=c_{3, k l}^{\prime}=0$ gives $\bar{F}_{k l}^{13^{\prime}}, c_{1, k l}^{\prime}=c_{2, k l}^{\prime}=0$ gives $\bar{F}_{k l}^{1^{\prime} 2^{\prime}}, c_{1, k l}^{\prime}=c_{3, k l}=0$ gives $\bar{F}_{k l}^{1^{\prime} 3}$, $c_{2, k l}=c_{3, k l}=0$ gives $\bar{F}_{k l}^{23}$ and $c_{2, k l}^{\prime}=c_{3, k l}^{\prime}=0$ gives $\bar{F}_{k l}^{2{ }^{\prime} 3^{\prime}}$. For $c_{1, k l}=c_{1, k l}^{\prime}=1$, the cubic equation gives that $c_{2, k l}^{\prime} c_{3, k l}=c_{2, k l} c_{3, k l}^{\prime}$, which implies that $\left(c_{3, k l}: c_{3, k l}^{\prime}\right)=\left(c_{3, k l}: \frac{c_{2, k l}^{\prime} c_{3, k l}}{c_{2, k l}}\right)=$ $\left(c_{2, k l}: c_{2, k l}^{\prime}\right)$, so we obtain $\bar{F}_{k l}^{11^{\prime}}$. In the same way $c_{2, k l}=c_{2, k l}^{\prime}=1$ gives $\bar{F}_{k l}^{22^{\prime}}$ and $c_{3, k l}=c_{3, k l}^{1}=1$ gives $\bar{F}_{k l}^{33^{\prime}}$.

The non-trivial intersections of these boundary sets are as follows:

$$
\begin{gathered}
\bar{F}_{k l}^{12} \cap \bar{F}_{k l}^{13^{\prime}}=((0: 1),(0: 1),(1: 0)), \bar{F}_{k l}^{12} \cap \bar{F}_{k l}^{23}=((0: 1),(0: 1),(0: 1)), \\
\bar{F}_{k l}^{13^{\prime}} \cap \bar{F}_{k l}^{2^{\prime} 3^{\prime}}=((0: 1),(1: 0),(1: 0)), \bar{F}_{k l}^{1^{\prime} 2^{\prime}} \cap \bar{F}_{k l}^{1^{\prime} 3}=((1: 0),(1: 0),(0: 1)), \\
\bar{F}_{k l}^{1^{\prime} 2^{\prime}} \cap \bar{F}_{k l}^{2^{\prime} 3^{\prime}}=((1: 0),(1: 0),(1: 0)), \bar{F}_{k l}^{1^{\prime} 3} \cap \bar{F}_{k l}^{23}=((1: 0),(0: 1),(0: 1)), \\
\bar{F}_{k l}^{1^{\prime} 2^{\prime}} \cap \bar{F}_{k l}^{33^{\prime}}=((1: 0),(1: 0),(1: 1)), \bar{F}_{k l}^{23} \cap \bar{F}_{k l}^{11^{\prime}}=((1: 1),(0: 1),(0: 1)), \\
\bar{F}_{k l}^{2^{\prime} 3^{\prime}} \cap \bar{F}_{k l}^{11^{\prime}}=((1: 1),(1: 0),(1: 0)), \bar{F}_{k l}^{11^{\prime}} \cap \bar{F}_{k l}^{22^{\prime}} \cap \bar{F}_{k l}^{33^{\prime}}=((1: 1),(1: 1),(1: 1)) .
\end{gathered}
$$

7.2. The space $\tilde{\mathcal{F}}$ as the blowup of $\mathcal{F}$. In order to resolve the problem indicated in Remark 6.8 we will blowup the cubic surface $\bar{F}_{i j}$ at the point $((1: 1),(1: 1),(1: 1))$. We denote by $\mathcal{F}$ the hypersurface in $\mathbb{C} P^{1} \times \mathbb{C} P^{1} \times \mathbb{C} P^{1}$ defined by

$$
\mathcal{F}=\left\{\left(\left(c_{1}: c_{1}^{\prime}\right),\left(c_{2}: c_{2}^{\prime}\right),\left(c_{3}: c_{3}^{\prime}\right)\right) \mid c_{1} c_{2}^{\prime} c_{3}=c_{1}^{\prime} c_{2} c_{3}^{\prime}\right\}
$$

Since the gradient of the cubic equation $c_{1} c_{2}^{\prime} c_{3}=c_{1}^{\prime} c_{2} c_{3}^{\prime}$, which defines this hypersurface, is nonzero, it holds:

Lemma 7.2. $\mathcal{F}$ is a smooth manifold.

We prove the following:

Theorem 7.3. An universal space of parameters $\tilde{\mathcal{F}}$ for $G_{5,2}$ can be obtained as the blowup of $\mathcal{F}$ at the point $((1: 1),(1: 1),(1: 1))$.

Before to proceed with the proof of Theorem 7.3 let us discuss the space $\tilde{\mathcal{F}}$ in more detail.

Corollary 7.4. $\tilde{\mathcal{F}}$ is a smooth compact four-manifold.

We describe this blowup in an neighborhood of the point $((1: 1),(1: 1),(1: 1))$.

Lemma 7.5. The blowup of the complex surface $\mathcal{F}=\left\{\left(\left(c_{1}: c_{1}^{\prime}\right),\left(c_{2}: c_{2}^{\prime}\right),\left(c_{3}: c_{3}^{\prime}\right)\right), c_{1} c_{2}^{\prime} c_{3}=\right.$ $\left.c_{1}^{\prime} c_{2} c_{3}^{\prime}\right\}$ in an neighborhood of the point $((1: 1),(1: 1),(1: 1))$ is the surface $\tilde{\mathcal{F}} \subset \mathcal{F} \times \mathbb{C} P^{1}$ defined by the equation:

$$
\left(1-c_{1}^{\prime}\right) x_{2}=\left(1-c_{2}^{\prime}\right) x_{1}
$$

where $\left(x_{1}: x_{2}\right) \in \mathbb{C} P^{1}$. 
Proof. We proceed in a standard way for doing the blowup at the point $((1: 1),(1: 1),(1: 1))$. Consider a neighborhood $U$ of this point of the form $\left(\left(1: c_{1}^{\prime}\right),\left(1: c_{2}^{\prime}\right),\left(1: c_{3}^{\prime}\right)\right)$, where $c_{1}^{\prime} \neq 0$. The cubic equation implies that $c_{2}^{\prime}=c_{1}^{\prime} c_{3}^{\prime}$, so, since we assume $c_{1}^{\prime} \neq 0$ in this neighborhood, we can take $\left(c_{1}^{\prime}, c_{2}^{\prime}\right)$ as coordinates in $U$. Then the preimage of $U \backslash\{((1: 1),(1: 1),(1: 1))\}$ in $\tilde{\mathcal{F}} \backslash \mathbb{C} P^{1}$ is an open submanifold of $\mathcal{F} \times \mathbb{C} P^{1}$ given by the equation $\left(1-c_{1}^{\prime}\right) x_{2}=\left(1-c_{2}^{\prime}\right) x_{1}$. This proves (16).

Corollary 7.6. The open manifold $\tilde{\mathcal{F}} \backslash\left\{\left((0: 1),(0: 1),\left(c_{3}: c_{3}^{\prime}\right)\right)\right\}$ is given by the equation

$$
\left(c_{1}-c_{1}^{\prime}\right) c_{2} c^{\prime}=c_{1}\left(c_{2}-c_{2}^{\prime}\right) c .
$$

Remark 7.7. The blowup construction implies that the projection map on the first coordinate $\pi$ : $\tilde{\mathcal{F}} \rightarrow \mathcal{F}$ is an isomorphism between $\tilde{\mathcal{F}} \backslash\{((1: 1),(1: 1),(1: 1))\} \times \mathbb{C} P^{1}$ and $\mathcal{F} \backslash\{((1: 1),(1:$ $1),(1: 1))\}$, and $\pi^{-1}((1: 1),(1: 1),(1: 1))=\mathbb{C} P^{1}$.

Remark 7.8. In the sequel, we write the points from $\tilde{\mathcal{F}} \backslash \mathbb{C} P^{1}$ in coordinates of the manifold $\mathcal{F}$ and the points from the divisor $\mathbb{C} P^{1} \subset \tilde{\mathcal{F}}$ in the form $\left(((1: 1),(1: 1),(1: 1)),\left(c_{3}: c_{3}^{\prime}\right)\right)$.

Theorem 7.9. The universal space of parameters $\tilde{\mathcal{F}}$ is homeomorphic to the space $Y$ which is the blowup of $\mathbb{C} P^{1} \times \mathbb{C} P^{1}$ at the points $B_{1}=((1: 0),(1: 0)), B_{2}=((0: 1),(0: 1))$ and $B_{3}=((1: 1),(1: 1))$.

Proof. The homeomorphism $\tilde{\mathcal{F}} \rightarrow Y$ is given by $\left(\left(c_{1}: c_{1}^{\prime}\right),\left(c_{2}: c_{2}^{\prime}\right),\left(c_{3}: c_{3}^{\prime}\right)\right) \rightarrow\left(\left(c_{1}: c_{1}^{\prime}\right),\left(c_{2}:\right.\right.$ $\left.\left.c_{2}^{\prime}\right)\right)$ at the points $\left(\left(c_{1}: c_{1}^{\prime}\right),\left(c_{2}: c_{2}^{\prime}\right),\left(c_{3}: c_{3}^{\prime}\right)\right)$ for which $\left(\left(c_{1}: c_{1}^{\prime}\right),\left(c_{2}: c_{2}^{\prime}\right)\right) \neq B_{1}, B_{2}, B_{3}$. For the other points, this homeomorphism is given by $\left((1: 0),(1: 0),\left(c_{3}: c_{3}^{\prime}\right)\right) \rightarrow\left(c_{3}: c_{3}^{\prime}\right) \in$ $\operatorname{blowup}\left(B_{1}\right) \cong \mathbb{C} P^{1},\left((0: 1),(0: 1),\left(c_{3}: c_{3}^{\prime}\right)\right) \rightarrow\left(c_{3}: c_{3}^{\prime}\right) \in \operatorname{blowup}\left(B_{2}\right) \cong \mathbb{C} P^{1}$ and $\left((1: 1),(1: 1),(1: 1),\left(c_{3}: c_{3}^{\prime}\right)\right) \rightarrow\left(c_{3}: c_{3}^{\prime}\right) \in \operatorname{blowup}\left(B_{3}\right) \cong \mathbb{C} P^{1}$.

We provide an interpretation of the universal space of parameters in one more way. Let

$$
U=\left(\mathbb{C} P^{1} \times \mathbb{C} P^{1}\right) \backslash\left\{\left(\left(c_{1}: c_{1}^{\prime}\right),(1: 0)\right),\left((1: 0),\left(c_{2}: c_{2}^{\prime}\right)\right)\right\}, \quad V=\mathbb{C} P^{2} \backslash\{(x: y: 0)\} .
$$

The following obviously holds:

Lemma 7.10. The map

$$
f: \mathbb{C} P^{1} \times \mathbb{C} P^{1} \longrightarrow \mathbb{C} P^{2}, f\left(\left(c_{1}: c_{1}^{\prime}\right),\left(c_{2}: c_{2}^{\prime}\right)\right)=\left(c_{1} c_{2}^{\prime}: c_{1}^{\prime} c_{2}: c_{1}^{\prime} c_{2}^{\prime}\right)
$$

has the following properties:

(1) $f$ is defined everywhere except and the point ((1:0), (1:0)).

(2) The image of $f$ is $V$.

(3) The space of parameters $F$ of the main stratum belongs to $U$.

(4) $f$ defines a homeomorphism $f: U \rightarrow V$.

Lemma 7.10 implies that the map $f$ induces a homeomorphism between the blowup of $\mathbb{C} P^{1} \times$ $\mathbb{C} P^{1}$ at three points and the blow up of $\mathbb{C} P^{2}$ at four points:

Corollary 7.11. The universal space of parameters $\tilde{\mathcal{F}}$ is homeomorphic to the space $Y$ obtained as the blowup of $\mathbb{C} P^{2}$ at four points $(1: 0: 0),(0: 1: 0),(0: 0: 1),(1: 1: 1)$.

Proof. Let us consider the blowup $\mathbb{C} P^{1} \times \mathbb{C} P^{1}$ at the point $((1: 0),(1: 0))$. We extend the map (17) to the map $f$ from this blowup to $\mathbb{C} P^{2}$ by mapping the blowup $((1: 0),(1: 0)) \cong \mathbb{C} P^{1}$ to $(x: y: 0) \subset \mathbb{C} P^{2}$. Then we consider the blowups of $\mathbb{C} P^{2}$ at the points $(1: 0: 0)$ and $(0: 1: 0)$ 
and map to these blowups the sets $\left\{\left((1: 0),\left(c_{2}: c_{2}^{\prime}\right)\right)\right\}$ and $\left\{\left(\left(c_{1}: c_{1}^{\prime}\right),(1: 0)\right)\right\}$, respectively. We obtain a homeomorphism between the blowup of $\mathbb{C} P^{1} \times \mathbb{C} P^{1}$ at one point and the blowup of $\mathbb{C} P^{2}$ at two points. Now, in addition, we do the blowup of $\mathbb{C} P^{1} \times \mathbb{C} P^{1}$ at the points $((0: 1),(0: 1))$ and $((1: 1),(1: 1))$ and obtain the universal space of parameters. We accordingly do the blowup of $\mathbb{C} P^{2}$ at the points $(0: 0: 1)$ and $(1: 1: 1)$ and map to them these two new blowups of $\mathbb{C} P^{1} \times \mathbb{C} P^{1}$. As a result we obtain the homeomorphism between the blowup of $\mathbb{C} P^{1} \times \mathbb{C} P^{1}$ at three points and the blowup of $\mathbb{C} P^{2}$ at four points.

Remark 7.12. The product $\mathbb{C} P^{1} \times \mathbb{C} P^{1}$ can be embedded into $\mathbb{C} P^{3}$ by the Serge map. It follows from (17) that there exists a mapping from $\mathbb{C} P^{1} \times \mathbb{C} P^{1}$ to $\mathbb{C} P^{2}$ which is not defined at one point. The proof of Corollary 7.11 shows that, after blowing up of $\mathbb{C} P^{1} \times \mathbb{C} P^{1}$ at that undefined point and blowing up $\mathbb{C} P^{2}$ at two points, we obtain a homeomorphism of these new spaces. We want to point that this is a special case of the result from toric topology [9], which states that the blowup of $\mathbb{C} P^{1} \times \mathbb{C} P^{1}$ at $k$ points is homeomorphic to the blowup of $\mathbb{C} P^{2}$ at $k+1$ points for $k>1$. The proof of Corollary 7.11 provides an explicit description of this homeomorphism for $k=2$.

Remark 7.13. In his seminal paper Kapranov [15] defined and studied the properties of the Chow quotient $G_{n, k} / /\left(\mathbb{C}^{*}\right)^{n}$. It is established an isomorphism between $G_{n, 2} / /\left(\mathbb{C}^{*}\right)^{n}$ and the GrothendieckKnudsen moduli space $\overline{M_{0, n}}$. Moreover, it is proved (Theorem 4.3.3, [15]) that the variety $\overline{M_{0, n}}$ can be obtained from $\mathbb{C} P^{n-3}$ by series of blowups. In the first non-trivial case when $n=5$, it implies that $\overline{M_{0,5}}$ is the blowup of $\mathbb{C} P^{2}$ at four points. In addition, as it is pointed in [15], in the paper [18] one can find a representation of $\overline{M_{0, n}}$ as an iterated blowup of $\left(\mathbb{C} P^{1}\right)^{n-3}$. From this representation (see [18], page 555), it directly follows that $\overline{M_{0,5}}$ is isomorphic to a blow up of $\mathbb{C} P^{1} \times \mathbb{C} P^{1}$ at three points. Thus, by Theorem 7.9 and Corollary 7.11 we see in both ways that the universal space of parameters $\tilde{\mathcal{F}}$ for $G_{5,2}$ is isomorphic to the Chow quotient $G_{5,2} / /\left(\mathbb{C}^{*}\right)^{5}$.

\section{THE TRANSITION AUTOMORPHISMS FOR THE SPACES OF PARAMETERS OF THE STRATA}

Consider the chart $M_{k l}$ and let $z_{i}^{k l}, 1 \leq i \leq 6$ be the coordinates in this chart. The main stratum in this chart is given by the system of equations

$$
c_{1, k l}^{\prime} z_{1}^{k l} z_{5}^{k l}=c_{1, k l} z_{2}^{k l} z_{4}^{k l}, \quad c_{2, k l}^{\prime} z_{1}^{k l} z_{6}^{k l}=c_{2, k l} z_{3}^{k l} z_{4}^{k l}, \quad c_{3, k l}^{\prime} z_{2}^{k l} z_{6}^{k l}=c_{3 . k l} z_{3}^{k l} z_{5}^{k l} .
$$

The set of parameters $F_{k l} \cong F$ of the main stratum in the coordinates of the chart $M_{k l}$ is given by

$$
\begin{gathered}
F_{k l}=\left\{\left(\left(c_{1, k l}: c_{1, k l}^{\prime}\right),\left(c_{2, k l}: c_{2, k l}^{\prime}\right),\left(c_{3, k l}: c_{3, k l}^{\prime}\right)\right) \in \mathbb{C} P^{1} \times \mathbb{C} P^{1} \times \mathbb{C} P^{1}\right\}, \\
c_{i, k l}, c_{i, k l}^{\prime} \neq 0, \quad c_{i, k l} \neq c_{i, k l}^{\prime}, \quad c_{1, k l} c_{2, k l}^{\prime} c_{3, k l}=c_{1, k l}^{\prime} c_{2, k l} c_{3, k l}^{\prime} .
\end{gathered}
$$

We have that

$$
c_{3, k l}^{\prime}=\frac{c_{1, k l} c_{2, k l}^{\prime}}{c_{1, k l}^{\prime} c_{2, k l}} c_{3, k l}
$$

which implies

$$
\left(c_{3, k l}: c_{3, k l}^{\prime}\right)=\left(c_{3, k l}: \frac{c_{1, k l} c_{2, k l}^{\prime}}{c_{1, k l}^{\prime} c_{2, k l}} c_{3, k l}\right)=\left(c_{1, k l}^{\prime} c_{2, k l}: c_{1, k l} c_{2, k l}^{\prime}\right) .
$$

Thus, we can take

$$
c_{3, k l}=c_{1, k l}^{\prime} c_{2, k l}, \quad c_{31}^{\prime}=c_{1, k l} c_{2, k l}^{\prime} .
$$


8.1. The transtion automorphisms for the space of parameters $F$ of the main stratum. Consider now the charts $M_{12}$ and $M_{13}$.

Proposition 8.1. The homeomorphism $f_{12,13}: F_{12} \rightarrow F_{13}$ is given by

$$
\left(\left(c_{1,12}: c_{1,12}^{\prime}\right),\left(c_{2,12}: c_{2,12}^{\prime}\right),\left(c_{3,12}: c_{3,12}^{\prime}\right)\right) \rightarrow
$$

$$
\rightarrow\left(\left(c_{1,12}: c_{1,12}-c_{1,12}^{\prime}\right),\left(c_{2,12}: c_{2,12}-c_{2,12}^{\prime}\right),\left(\left(c_{1,12}-c_{1,12}^{\prime}\right) c_{2,12}^{\prime} c_{3,12}: c_{1,12}^{\prime}\left(c_{2,12}-c_{2,12}^{\prime}\right) c_{3,12}^{\prime}\right)\right) .
$$

Proof. The coordinates $z_{i}^{12}$ and $z_{i}^{13}, 1 \leq i \leq 6$ are, on $M_{12} \cap M_{13}$, related by

$$
\begin{gathered}
z_{1}^{13}=-\frac{z_{1}^{12}}{z_{4}^{12}}, \quad z_{2}^{13}=z_{2}^{12}-\frac{z_{1}^{12}}{z_{4}^{12}} z_{5}^{12}, \quad z_{3}^{13}=z_{3}^{12}-\frac{z_{1}^{12}}{z_{4}^{12}} z_{6}^{12} . \\
z_{4}^{13}=\frac{1}{z_{4}^{12}}, \quad z_{5}^{13}=\frac{z_{5}^{12}}{z_{4}^{12}}, \quad z_{6}^{13}=\frac{z_{6}^{12}}{z_{4}^{12}} .
\end{gathered}
$$

It follows that

$$
\begin{gathered}
z_{1}^{13} z_{5}^{13}=-\frac{z_{1}^{12} z_{5}^{12}}{\left(z_{4}^{12}\right)^{2}}, \quad z_{2}^{13} z_{4}^{13}=\frac{z_{2}^{12} z_{4}^{12}-z_{1}^{12} z_{5}^{12}}{\left(z_{4}^{12}\right)^{2}}, \quad z_{1}^{13} z_{6}^{13}=-\frac{z_{1}^{12} z_{6}^{12}}{\left(z_{4}^{12}\right)^{2}} \\
z_{3}^{13} z_{4}^{13}=\frac{z_{3}^{12} z_{4}^{12}-z_{1}^{12} z_{6}^{12}}{\left(z_{4}^{12}\right)^{2}}, \quad z_{2}^{13} z_{6}^{13}=z_{6}^{12} \frac{z_{2}^{12} z_{4}^{12}-z_{1}^{12} z_{5}^{12}}{\left(z_{4}^{12}\right)^{2}}, \quad z_{3}^{13} z_{5}^{13}=z_{5}^{12} \frac{z_{3}^{12} z_{4}^{12}-z_{1}^{12} z_{6}^{12}}{\left(z_{4}^{12}\right)^{2}}
\end{gathered}
$$

Therefore, the relation $c_{1,13}^{\prime} z_{1}^{13} z_{5}^{13}=c_{1,13} z_{2}^{13} z_{4}^{13}$ can be written as

$$
c_{1,13}^{\prime} z_{1}^{12} z_{5}^{12}=c_{1,13}\left(z_{1}^{12} z_{5}^{12}-z_{2}^{12} z_{5}^{12}\right) \Rightarrow\left(c_{1,13}-c_{1,13}^{\prime}\right) z_{1}^{12} z_{5}^{12}=c_{1,13} z_{2}^{12} z_{4}^{12} .
$$

It follows from (18) that

$$
c_{1,13}=c_{1,12}, \quad c_{1,13}^{\prime}=c_{1,12}-c_{1,12}^{\prime} .
$$

In the same way we deduce that

$$
c_{2,13}=c_{2,12}, \quad c_{2,13}^{\prime}=c_{2,12}-c_{2,12}^{\prime} .
$$

From (18) we also deduce that

$$
\frac{c_{1, k l}}{c_{1, k l}^{\prime}}=\frac{z_{1}^{k l} z_{5}^{k l}}{z_{2}^{k l} z_{4}^{k l}}, \quad \frac{c_{2, k l}}{c_{2, k l}^{\prime}}=\frac{z_{1}^{k l} z_{6}^{k l}}{z_{3}^{k l} z_{4}^{k l}}, \quad \frac{c_{3, k l}}{c_{3, k l}^{\prime}}=\frac{z_{2}^{k l} z_{6}^{k l}}{z_{3}^{k l} z_{5}^{k l}} .
$$

Taking into account (23), it follows that

$$
\begin{gathered}
c_{3,13}^{\prime}=\frac{z_{5}^{12}}{z_{6}^{12}} \frac{z_{3}^{12} z_{4}^{12}-z_{1}^{12} z_{6}^{12}}{z_{2}^{12} z_{4}^{12}-z_{1}^{12} z_{5}^{12}} c_{3,13}= \\
\frac{z_{5}^{12}}{z_{6}^{12}} \frac{z_{3}^{12} z_{4}^{12}}{z_{2}^{12} z_{4}^{12}} \frac{\frac{c_{2,12}}{c_{2,12}^{\prime}}-1}{\frac{c_{1,12}}{c_{2,12}^{\prime}}-1} c_{3,13}=\frac{c_{1,12}^{\prime}\left(c_{2,12}-c_{2,12}^{\prime}\right) c_{3,12}^{\prime}}{\left(c_{1,12}-c_{1,12}^{\prime}\right) c_{2,12}^{\prime} c_{3,12}} c_{3,13} .
\end{gathered}
$$

Thus,

$$
\left(c_{3,13}: c_{3,13}^{\prime}\right)=\left(\left(c_{1,12}-c_{1,12}^{\prime}\right) c_{2,12}^{\prime} c_{3,12}: c_{1,12}^{\prime}\left(c_{2,12}-c_{2,12}^{\prime}\right) c_{3,12}^{\prime}\right) .
$$


Due to the fact that the group $S_{5}$ permutes the charts it can be similarly explicitly constructed a homeomorphism $f_{i j, k l}: F_{i j} \rightarrow F_{k l}$ between the sets of parameters $F_{i j}$ and $F_{k l}$ of the main stratum for an arbitrary two charts $M_{i j}$ and $M_{k l}$.

Since the homeomorphism $f_{i j, k l}: F_{i j} \rightarrow F_{k l}$ is induced by the coordinate transition map for the charts $M_{i j}$ and $M_{k l}$, we deduce:

Lemma 8.2. For any three charts $M_{i j}, M_{k l}$ and $M_{m n}$ it holds

$$
f_{i j, k l}=f_{m n, k l} \circ f_{i j, m n} .
$$

Corollary 8.3. The automorphisms $f_{i j, k l}$ of the space of parameters $F$ of the main stratum for $G_{5,2}$, which are induced by the coordinate transition maps between all charts, form a group. The generators for this group are given by the set $\left\{f_{i_{0} j_{0}, k l}, i_{0} j_{0} \neq k l\right\}$ for any fixed $i_{0} j_{0}$.

8.2. The transition automorphisms for the spaces of parameters of other non one-orbit strata. Let $W_{\sigma}$ be a non-orbit stratum, which is different from the main stratum. The stratum $W_{\sigma}$ does not belong to all charts. We determine here the relation between the coordinate records of the set of parameters $F_{\sigma}$ for $W_{\sigma}$ in different charts which contain $W_{\sigma}$. We demonstrate this for one stratum, which belongs to the charts $M_{12}$ and $M_{13}$. Due to an action of $S_{5}$, the similar relation will hold for an arbitrary stratum and arbitrary charts. The intersections of the charts $M_{12}$ and $M_{13}$ is, in the coordinates of these charts, given by the condition $z_{4}^{12} \neq 0$ and $z_{4}^{13} \neq 0$. Consider the stratum whose admissible polytope is $P_{\sigma}=K_{34}(9)$, which belongs to the both of these charts. The stratum $W_{\sigma}$ is, in the chart $M_{12}$, given by

$$
\begin{gathered}
z_{1}^{12} z_{5}^{12}=z_{2}^{12} z_{4}^{12}, \quad c_{2,12}^{\prime} z_{1}^{12} z_{6}^{12}=c_{2,12} z_{3}^{12} z_{4}^{12}, \quad c_{3,12}^{\prime} z_{2}^{12} z_{6}^{12}=c_{3,12} z_{3}^{12} z_{5}^{12}, \\
c_{2,12}, c_{2,12}^{\prime} \neq 0, \quad c_{2,12} \neq c_{2,12}^{\prime}, \quad c_{3,12}, c_{3,12}^{\prime} \neq 0, \quad c_{3,12} \neq c_{3,12}^{\prime} . \quad c_{2,12}^{\prime} c_{3,12}=c_{2,12} c_{3,12}^{\prime} .
\end{gathered}
$$

It follows that

$$
c_{3,12}^{\prime}=\frac{c_{2,12}^{\prime} c_{3,12}}{c_{2,12}},
$$

which implies

$$
\left(c_{3,12}: c_{3,12}^{\prime}\right)=\left(c_{2,12}: c_{2,12}^{\prime}\right)=\left(c_{12}: c_{12}^{\prime}\right) .
$$

Thus, the set of parameters for the stratum $W_{\sigma}$ is, in the coordinates for $M_{12}$, given by

$$
F_{\sigma, 12}=\left\{\left(c_{12}: c_{12}^{\prime}\right), \quad c_{12}, c_{12}^{\prime} \neq 0, \quad c_{12} \neq c_{12}^{\prime}\right\} .
$$

The stratum $W_{\sigma}$ is, in the chart $M_{13}$, given by the equations:

$$
z_{2}^{13}=0, \quad c_{2,13}^{\prime} z_{1}^{13} z_{6}^{13}=c_{2,13} z_{3}^{13} z_{4}^{13}, \quad c_{2,13}, c_{2,13}^{\prime} \neq 0, \quad c_{2,13} \neq c_{2,13}^{\prime} .
$$

Thus, the set of parameters for $W_{\sigma}$ is, in the chart $M_{13}$, given by

$$
F_{\sigma, 13}=\left\{\left(c_{13}: c_{13}^{\prime}\right), \quad c_{13}, c_{13}^{\prime} \neq 0, \quad c_{13} \neq c_{13}^{\prime}\right\} .
$$

Substituting the formulas (22) into (24) we obtain that a homeomorphism between $F_{\sigma, 12}$ and $F_{\sigma, 13}$ is given by:

$$
\left(c_{12}: c_{12}^{\prime}\right) \rightarrow\left(c_{12}: c_{12}-c_{12}^{\prime}\right) .
$$

Due to an action of the group $S_{5}$, a homeomorphism $f_{\sigma, i j, k l}: F_{\sigma, i j} \rightarrow F_{\sigma, k l}$ between the sets of parameters $F_{\sigma, i j}$ and $F_{\sigma, k l}$ of a stratum $W_{\sigma}$ can be explicitly constructed for an arbitrary two charts $M_{i j}$ and $M_{k l}$ such that $W_{\sigma} \subset M_{i j}, M_{k l}$. 
Lemma 8.4. For any three charts $M_{i j}, M_{k l}$ and $M_{m n}$ such that $W_{\sigma} \subset M_{i j}, M_{k l}, M_{m n}$, it holds

$$
f_{\sigma, i j, k l}=f_{\sigma, i j, m n} \circ f_{\sigma, m n, k l} .
$$

Corollary 8.5. The automorphisms $f_{\sigma, i j, k l}$ of the space of parameters $F_{\sigma}$ of a stratum $W_{\sigma}$, which are induced by the coordinate transition maps between the charts which contain the stratum $W_{\sigma}$, form a group.

\section{THE PROOF OF THEOREM 5.1}

Taking into account Remark 6.8, we first prove that the set $\tilde{\mathcal{F}}$ given by Theorem 7.3 is the universal set of parameters in the following sense. Denote by $\tilde{\mathcal{F}}_{i j}$ the coordinate record of $\tilde{\mathcal{F}}$ in a chart $M_{i j}$. If $W_{\sigma} \subset M_{i j}$, denote by $\tilde{F}_{\sigma, i j} \subset \tilde{\mathcal{F}}_{i j}$ a subset defined by $c \in \tilde{F}_{\sigma, i j}$ if and only if there exists a sequence $\left(x_{n}, c_{n}\right) \in \stackrel{\circ}{\Delta}, 2_{5,2} \times F_{i j}$ such that $c_{n} \rightarrow c$ in $\tilde{\mathcal{F}}_{i j}$ and $h^{-1}\left(x_{n}, f_{i j}\left(c_{n}\right)\right)$ converges to a point from $W_{\sigma} / T^{5}$, for the earlier defined homeomoprhisms $f_{i j}: F_{i j} \rightarrow F$ and $h: W / T^{n} \rightarrow \stackrel{\circ}{\Delta}_{5,2}$.

Theorem 9.1. For an arbitrary charts $M_{i j}$ and $M_{k l}$, there is a homeomorphism $\tilde{f}_{i j, k l}: \tilde{\mathcal{F}}_{i j} \rightarrow \tilde{\mathcal{F}}_{k l}$ such that

(1) $\tilde{f}_{i j, k l}=f_{i j, k l}$ on $F_{i j}$,

(2) for any stratum $W_{\sigma} \subset M_{i j} \cap M_{k l}$ it holds $\tilde{f}_{i j, k l}\left(\tilde{F}_{\sigma, i j}\right)=\tilde{F}_{\sigma, k l}$.

Since $\tilde{\mathcal{F}}_{i j} \cong \tilde{\mathcal{F}}$ for any $\{i, j\} \subset\{1, \ldots, 5\}$, Theorem 9.1 implies:

Corollary 9.2. The automorphisms $\tilde{f}_{i j, k l}$ of the universal space of parameters $\tilde{\mathcal{F}}$, which are induced by the coordinate transition maps between the charts, form a group.

It is enough to prove Theorem 9.1 for the charts $M_{12}$ and $M_{13}$, since all our arguments are compatible with an action of the group $S_{5}$. We need to prove that the homeomorphism $f_{12,13}$ : $F_{12} \rightarrow F_{13}$ given by

$$
\begin{gathered}
\left(\left(c_{1,12}: c_{1,12}^{\prime}\right),\left(c_{2,12}: c_{2,12}^{\prime}\right),\left(c_{3,12}: c_{3,12}^{\prime}\right)\right) \rightarrow \\
\rightarrow\left(\left(c_{1,12}: c_{1,12}-c_{1,12}^{\prime}\right),\left(c_{2,12}: c_{2,12}-c_{2,12}^{\prime}\right),\left(\left(c_{1,12}-c_{1,12}^{\prime}\right) c_{2,12}^{\prime} c_{3,12}: c_{1,12}^{\prime}\left(c_{2,12}-c_{2,12}^{\prime}\right) c_{3,12}^{\prime}\right)\right),
\end{gathered}
$$

can be extended to a homeomorphism $\tilde{f}_{12,13}: \tilde{\mathcal{F}}_{12} \rightarrow \tilde{\mathcal{F}}_{13}$. We proceed with the proof through the following lemmas.

Lemma 9.3. The homeomorphism $f_{12,13}$ is defined on $\bar{F}_{12,13}$ except on the sets $\bar{F}_{12}^{1^{\prime} 2^{\prime}}, \bar{F}_{12}^{2^{\prime} 3^{\prime}}, \bar{F}_{12}^{1^{\prime} 3}$, 1), (1:1), (1:1)).

Proof. . It follows directly from the definition of $f_{12,13}$ that $f_{12,13}$ is not defined in the following cases: $c_{1}=c_{1}^{\prime}, c_{2}=c_{2}^{\prime} ; c_{1}=c_{1}^{\prime}, c_{3}^{\prime}=0 ; c_{1}^{\prime}=c_{2}^{\prime}=0 ; c_{2}^{\prime}=c_{3}^{\prime}=0 ; c_{1}^{\prime}=c_{3}=0 ; c_{2}=c_{2}^{\prime}, c_{3}=0$. Note that the relation $c_{1} c_{2}^{\prime} c_{3}=c_{1}^{\prime} c_{2} c_{3}^{\prime}$ gives that the sixth and the fifth case are the same, as well as the second and the forth case. Therefore, $f_{12,13}$ is not defined on the sets $\bar{F}_{k l}^{1^{\prime} 2^{\prime}}, \bar{F}_{k l}^{2^{\prime} 3^{\prime}}, \bar{F}_{k l}^{1^{\prime}}, \quad((1$ : $1),(1: 1),(1: 1))$.

Let us consider now the set $\bar{F}_{12}^{1^{\prime} 3}=(1: 0) \times \mathbb{C} P^{1} \times(0: 1)$. We prove that $f_{12,13}$ can be continuously extended to this set.

Lemma 9.4. The homeomorphism $f_{12,13}: F_{12} \rightarrow F_{13}$ can be continuously extended to a homeomorphism $\bar{f}_{12,13}^{1^{3} 3}: \bar{F}_{12}^{1^{\prime} 3} \rightarrow \bar{F}_{13}^{11^{\prime}}$ by $\bar{f}_{12,13}^{1^{\prime} 3}\left((1: 0),\left(c_{2}: c_{2}^{\prime}\right),(0: 1)\right)=\left((1: 1),\left(c_{2}: c_{2}-c_{2}^{\prime}\right),\left(c_{2}:\right.\right.$ $\left.c_{2}-c_{2}^{\prime}\right)$ ). 
Proof. Let us consider a point $c_{0}=\left((1: 0),\left(c_{2}: c_{2}^{\prime}\right),(0: 1)\right)$, where $c_{2} \neq 0$ and let $c(n)=$ $\left(\left(c_{1,12}(n): c_{1,12}^{\prime}(n)\right),\left(c_{2,12}(n): c_{2,12}^{\prime}(n)\right),\left(c_{3,12}(n): c_{3,12}^{\prime}(n)\right)\right)$ be a sequence of points from $F_{12}$ which converges to $c_{0} \in \mathbb{C} P^{1} \times \mathbb{C} P^{1} \times \mathbb{C} P^{1}$. It implies that

$$
\begin{gathered}
\left(c_{1,12}(n): c_{1,12}^{\prime}(n)\right) \rightarrow(1: 0) \Rightarrow\left(1: \frac{c_{1,12}^{\prime}(n)}{c_{1,12}(n)}\right) \rightarrow(1: 0) \Rightarrow \frac{c_{1,12}^{\prime}(n)}{c_{1,12}(n)} \rightarrow 0, \\
\left(c_{3,12}(n): c_{3,12}^{\prime}(n)\right) \rightarrow(0: 1) \Rightarrow\left(\frac{c_{3,12}(n)}{c_{3,12}^{\prime}(n)}: 1\right) \rightarrow(0: 1) \Rightarrow \frac{c_{3,12}(n)}{c_{3,12}^{\prime}(n)} \rightarrow 0, \\
\left(c_{2,12}(n): c_{2,12}^{\prime}(n)\right) \rightarrow\left(c_{2}: c_{2}^{\prime}\right) \Rightarrow\left(1: \frac{c_{2,12}^{\prime}(n)}{c_{2,12}(n)}\right) \rightarrow\left(1: \frac{c_{2}^{\prime}}{c_{2}}\right) \Rightarrow \frac{c_{2,12}^{\prime}(n)}{c_{2,12}(n)} \rightarrow \frac{c_{2}^{\prime}}{c_{2}} .
\end{gathered}
$$

Since $c(n) \in F_{12}$ we see that $f_{12,13}(c(n))$ is well defined and

$$
\begin{gathered}
\left(c_{1,12}(n): c_{1,12}(n)-c_{1,12}^{\prime}(n)\right)=\left(1: 1-\frac{c_{1,12}^{\prime}(n)}{c_{1,12}(n)}\right) \rightarrow(1: 1), \\
\left(c_{2,12}(n): c_{2,12}(n)-c_{2,12}(n)^{\prime}\right)=\left(1: 1-\frac{c_{2,12}^{\prime}(n)}{c_{2,12}(n)}\right) \rightarrow\left(1: 1-\frac{c_{2}^{\prime}}{c_{2}}\right) .
\end{gathered}
$$

Since $c(n) \in F_{12}$, it follows that $c(n)$ satisfies the cubic equation, so we obtain

$$
c_{1,12}^{\prime}(n) c_{3,12}^{\prime}(n)=\frac{c_{1,12}(n) c_{2,12}^{\prime}(n) c_{3,12}(n)}{c_{2,12}(n)} .
$$

It implies that

$$
\begin{gathered}
\left(\left(c_{1,12}^{\prime}(n)-c_{1,12}(n)\right) c_{2,12}^{\prime}(n) c_{3,12}(n): c_{1,12}^{\prime}(n)\left(c_{2,12}^{\prime}(n)-c_{2,12}(n)\right) c_{3,12}^{\prime}(n)\right)= \\
\left(\left(c_{1,12}^{\prime}(n)-c_{1,12}(n)\right) c_{2,12}^{\prime}(n) c_{3,12}(n): \frac{c_{1,12}(n) c_{2,12}^{\prime}(n) c_{3,12}(n)}{c_{2,12}(n)}\right)\left(c_{2,12}^{\prime}(n)-c_{2,12}(n)\right)= \\
\left(\left(c_{1,12}^{\prime}(n)-c_{1,12}(n): \frac{c_{1,12}(n)}{c_{2,12}(n)}\left(c_{2,12}^{\prime}(n)-c_{2,12}(n)\right)\right)=\left(1: \frac{1-\frac{c_{2,12}^{\prime}(n)}{c_{2,12}(n)}}{1-\frac{c_{1,12}^{\prime}(n)}{c_{1,12}(n)}}\right) \rightarrow\left(1: 1-\frac{c_{2}^{\prime}}{c_{2}}\right) .\right.
\end{gathered}
$$

In this way we conclude that

$$
h_{12,13}(c(n)) \rightarrow\left((1: 1),\left(1: 1-\frac{c_{2}^{\prime}}{c_{2}}\right),\left(1: 1-\frac{c_{2}^{\prime}}{c_{2}}\right)\right) .
$$

Therefore, we set

$$
\bar{f}_{12,13}^{1^{\prime} 3}\left((1: 0),\left(c_{2}: c_{2}^{\prime}\right),(0: 1)\right)=\left((1: 1),\left(1: 1-\frac{c_{2}^{\prime}}{c_{2}}\right),\left(1: 1-\frac{c_{2}^{\prime}}{c_{2}}\right)\right),
$$

where $c_{2} \neq 0$.

For $c_{2}=0$ we obtain the point $((1: 0),(0: 1),(0: 1))$ and, for a sequence $c(n)$ which converges to this point, it holds

$$
\frac{c_{1,12}^{\prime}(n)}{c_{1,12}(n)} \rightarrow 0, \frac{c_{2,12}(n)}{c_{2,12}^{\prime}(n)} \rightarrow 0, \frac{c_{3,12}(n)}{c_{3,12}^{\prime}(n)} \rightarrow 0
$$


If we consider the sequence $f_{12,13}(c(n))$ we obtain

$$
\begin{gathered}
\left(c_{1,12}(n): c_{1,12}(n)-c_{1,12}^{\prime}(n)\right)=\left(1: 1-\frac{c_{1,12}^{\prime}(n)}{c_{1,12}(n)}\right) \rightarrow(1: 1), \\
\left(c_{2,12}(n): c_{2,12}(n)-c_{2,12}^{\prime}(n)\right)=\left(\frac{c_{2,12}(n)}{c_{2,12}^{\prime}(n)}: \frac{c_{2,12}(n)}{c_{2,12}^{\prime}(n)}-1\right) \rightarrow(0: 1),
\end{gathered}
$$

Since $c(n) \in F_{12}$, it follows that $c(n)$ satisfies the cubic equation which implies

$$
c_{2,12}^{\prime}(n) c_{3,12}(n)=\frac{c_{1,12}^{\prime}(n) c_{2,12}^{\prime}(n) c_{3,12}^{\prime}(n)}{c_{1,12}(n)}
$$

Substituting this into the third coordinate for $f_{12,13}(c(n))$ we obtain

$$
\begin{gathered}
\left(\left(c_{1,12}^{\prime}(n)-c_{1,12}(n)\right) c_{2,12}^{\prime}(n) c_{3,12}(n): c_{1,12}^{\prime}(n)\left(c_{2,12}^{\prime}(n)-c_{2,12}(n)\right) c_{3,12}^{\prime}(n)\right)= \\
\left(\left(c_{1,12}^{\prime}(n)-c_{1,12}(n)\right) \frac{c_{1,12}^{\prime}(n) c_{2,12}(n) c_{3,12}^{\prime}(n)}{c_{1,12}(n)}: c_{1,12}(n) c_{3,12}^{\prime}(n)\left(c_{2,12}^{\prime}(n)-c_{2,12}(n)\right)\right)= \\
\left(\left(c_{1,12}^{\prime}(n)-c_{1,12}(n)\right) \frac{c_{1,12}^{\prime}(n) c_{2,12}(n)}{c_{1,12}(n)}: c_{1,12}(n)\left(c_{2,12}^{\prime}(n)-c_{2,12}(n)\right)\right)= \\
\left(\left(\frac{c_{1,12}^{\prime}(n)}{c_{1,12}(n)}-1\right) \frac{c_{1,12}^{\prime}(n)}{c_{1,12}(n)} c_{2,12}(n): c_{2,12}^{\prime}(n)-c_{2,12}(n)\right)= \\
\left(\left(\frac{c_{1,12}^{\prime}(n)}{c_{1,12}(n)}-1\right) \frac{c_{1,12}^{\prime}(n)}{c_{1,12}(n)} \frac{c_{2,12}(n)}{c_{2,12}^{\prime}(n)}: 1-\frac{c_{2,12}(n)}{c_{2,12}^{\prime}(n)}\right)= \\
\left(\frac{c_{1,12}(n)^{\prime}}{c_{1,12}(n)}-1\right) \frac{c_{1,12}^{\prime}(n)}{c_{1,12}(n)} \frac{c_{2,12}(n)}{c_{2,12}^{\prime}(n)} \\
1-\frac{c_{2,12}(n)}{c_{2,12}^{\prime}(n)}
\end{gathered}
$$

Therefore, we define

$$
\bar{f}_{12,13}^{1^{\prime} 3}((1: 0),(1: 0),(0: 1))=((1: 1),(0: 1),(0: 1))
$$

In a similar way it can be proved:

Lemma 9.5. The homeomorphism $f_{12,13}: F_{12} \rightarrow F_{13}$ can be continuously extended to a homeomorphism $\bar{f}_{12,13}^{2^{\prime} 3^{\prime}}: \bar{F}_{12}^{2^{\prime} 3^{\prime}} \rightarrow \bar{F}_{13}^{22^{\prime}}$ by $\bar{f}_{12,13}^{2^{\prime} 3^{\prime}}\left(\left(c_{1}: c_{1}^{\prime}\right),(1: 0),(1: 0)\right)=\left(\left(c_{1}: c_{1}-c_{1}^{\prime}\right),(1:\right.$ 1), $\left.\left(c_{1}-c_{1}^{\prime}: c_{1}\right)\right)$.

Proof. Let $c_{0}=\left(\left(c_{1}: c_{1}^{\prime}\right),(1: 0),(1: 0)\right)$ and let a sequence $c(n) \in F_{12}$ converges to $c_{0}$. It implies that

Therefore,

$$
\frac{c_{1}^{\prime}(n)}{c_{1}(n)} \rightarrow \frac{c_{1}^{\prime}}{c_{1}}, \frac{c_{2}^{\prime}(n)}{c_{2}(n)} \rightarrow 0, \frac{c_{3}^{\prime}(n)}{c_{3}(n)} \rightarrow 0
$$

$$
\begin{gathered}
\left(c_{1}(n): c_{1}(n)-c_{1}^{\prime}(n)\right) \rightarrow\left(1: 1-\frac{c_{1}^{\prime}}{c_{1}}\right), \quad\left(c_{2}(n): c_{2}(n)-c_{2}^{\prime}(n)\right) \rightarrow(1: 1), \\
\left(\left(c_{1,12}(n)-c_{1,12}^{\prime}(n)\right) c_{2,12}^{\prime}(n) c_{3,12}(n): c_{1,12}^{\prime}(n)\left(c_{2,12}(n)-c_{2,12}^{\prime}(n)\right) c_{3,12}^{\prime}(n)\right)=
\end{gathered}
$$




$$
\begin{gathered}
\left(\left(c_{1,12}(n)-c_{1,12}^{\prime}(n)\right) c_{2,12}^{\prime}(n) c_{3,12}(n): \frac{c_{1,12}(n) c_{2,12}^{\prime}(n) c_{3,12}(n)}{c_{2,12}(n)}\left(c_{2,12}(n)-c_{2,12}^{\prime}(n)\right)\right)= \\
\left(c_{1,12}(n)-c_{1,12}^{\prime}(n): \frac{c_{1,12}(n)}{c_{2,12}(n)}\left(c_{2,12}(n)-c_{2,12}^{\prime}(n)\right)\right)= \\
\left(1: \frac{1-\frac{c_{2,12}^{\prime}(n)}{c_{2,12}(n)}}{1-\frac{c_{1,12}^{\prime}(n)}{c_{1,12}(n)}}\right) \rightarrow\left(c_{1}-c_{1}^{\prime}: c_{1}\right) .
\end{gathered}
$$

Lemma 9.6. The homeomorphism $f_{12,13}: F_{12} \rightarrow F_{13}$ continuously extends to a constant map $\bar{f}_{12,13}^{1^{\prime} 2^{\prime}}: \bar{F}_{12}^{1^{\prime} 2^{\prime}} \rightarrow \bar{F}_{13}^{11^{\prime}} \cap \bar{F}_{13}^{22^{\prime}} \cap F_{13}^{33^{\prime}}$ by $\bar{f}_{12,13}^{1^{\prime} 2^{\prime}}\left((1: 0),(1: 0),\left(c_{3}: c_{3}^{\prime}\right)\right)=((1: 1),(1: 1),(1: 1))$.

Proof. Let $c_{0}=\left((1: 0),(1: 0),\left(c_{3}: c_{3}^{\prime}\right)\right)$ and let $c(n)$ be a sequence of points from $F_{12}$ which converges to $c_{0}$. It holds:

$$
\begin{gathered}
\left(c_{1,12}(n): c_{1,12}^{\prime}(n)\right) \rightarrow(1: 0) \Rightarrow \frac{c_{1.12}^{\prime}(n)}{c_{1,12}(n)} \rightarrow 0, \\
\left(c_{2,12}(n): c_{2,12}^{\prime}(n)\right) \rightarrow(1: 0) \Rightarrow \frac{c_{2,12}^{\prime}(n)}{c_{2,12}(n)} \rightarrow 0, \\
\left(c_{3,12}(n): c_{3,12}^{\prime}(n)\right) \rightarrow\left(c_{3}: c_{3}^{\prime}\right) \Rightarrow \frac{c_{3,12}^{\prime}(n)}{c_{3,12}(n)} \rightarrow \frac{c_{3}^{\prime}}{c_{3}} .
\end{gathered}
$$

It implies that

$$
\begin{gathered}
\left(c_{1,12}(n): c_{1,12}(n)-c_{1,12}^{\prime}(n)\right)=\left(1: 1-\frac{c_{1,12}^{\prime}(n)}{c_{1,12}(n)}\right) \rightarrow(1: 1), \\
\left(c_{2,12}(n): c_{2,12}(n)-c_{2,12}^{\prime}(n)\right)=\left(1: 1-\frac{c_{2,12}^{\prime}(n)}{c_{2,12}(n)}\right) \rightarrow(1: 1), \\
\left(\left(c_{1,12}(n)-c_{1,12}^{\prime}(n)\right) c_{2,12}^{\prime}(n) c_{3,12}(n): c_{1,12}^{\prime}(n)\left(c_{2,12}(n)-c_{2,12}^{\prime}(n)\right) c_{3,12}^{\prime}(n)\right)= \\
\left(\left(c_{1,12}(n)-c_{1,12}^{\prime}(n)\right) c_{2,12}^{\prime}(n) c_{3,12}(n): \frac{c_{1,12}(n) c_{2,12}^{\prime}(n) c_{3,12}(n)}{c_{2,12}(n)}\left(c_{2,12}(n)-c_{2,12}^{\prime}(n)\right)\right)= \\
\left(c_{1,12}(n)-c_{1,12}^{\prime}(n): \frac{c_{1,12}(n)}{c_{2,12}(n)}\left(c_{2,12}(n)-c_{2,12}^{\prime}(n)\right)\right)=\left(1: \frac{1-\frac{c_{2,12}^{\prime}(n)}{c_{2,12}(n)}}{1-\frac{c_{1,12}^{\prime}(n)}{c_{1,12}(n)}}\right) \rightarrow(1: 1) .
\end{gathered}
$$

Therefore, the homeomorphism $f_{12,13}$ continuously extends to $\bar{f}_{12,13}^{1^{\prime} 2^{\prime}}: \bar{F}_{12}^{1^{\prime} 2^{\prime}} \rightarrow((1: 1),(1: 1),(1:$ 1)) $=\bar{F}_{13}^{11^{\prime}} \cap \bar{F}_{13}^{22^{\prime}} \cap \bar{F}_{13}^{33^{\prime}}$.

Lemma 9.7. The homeomorphism $f_{12,13}: F_{12} \rightarrow F_{13}$ can not be continuously extended to the point $((1: 1),(1: 1),(1: 1)) \in \bar{F}_{12}$. 
Proof. Let us consider the point $((1: 1),(1: 1),(1: 1))$ and a sequence $c(n) \in F_{12}$ which converges to this point. Then

$$
\begin{gathered}
\left(c_{1,12}(n): c_{1,12}(n)-c_{1,12}^{\prime}(n)\right)=\left(1: 1-\frac{c_{1,12}^{\prime}(n)}{c_{1,12}(n)}\right) \rightarrow(1: 0), \\
\left(c_{2,12}(n): c_{2,12}(n)-c_{2,12}^{\prime}(n)\right)=\left(1: 1-\frac{c_{2,12}^{\prime}(n)}{c_{2,12}(n)}\right) \rightarrow(1: 0), \\
\left(\left(c_{1,12}(n)-c_{1,12}^{\prime}(n)\right) c_{2,12}^{\prime}(n) c_{3,12}(n): c_{1,12}^{\prime}(n)\left(c_{2,12}(n)-c_{2,12}^{\prime}(n)\right) c_{3,12}^{\prime}(n)\right)= \\
\left(c_{1,12}(n)-c_{1,12}^{\prime}(n): \frac{c_{1,12}(n)}{c_{2,12}(n)}\left(c_{2,12}(n)-c_{2,12}^{\prime}(n)\right)\right)=\left(1: \frac{1-\frac{c_{2,12}^{\prime}(n)}{c_{2,12}(n)}}{1-\frac{c_{1,12}^{\prime}(n)}{c_{1,12}(n)}}\right) .
\end{gathered}
$$

Since $\frac{c_{1,12}^{\prime}(n)}{c_{1,12}(n)} \rightarrow 1$ and $\frac{c_{2,12}^{\prime}(n)}{c_{2,12}(n)} \rightarrow 1$ we deduce that the above limit is not defined, so $f_{12,13}$ can not be continuously extended to the point $((1: 1),(1 ; 1),(1: 1))$.

Remark 9.8. As Lemma 9.4 and Lemma 9.5 show the problem with an extension of the homeomorphism $f_{12,13}: F_{12} \rightarrow F_{13}$ to the boundary of $F_{12}$ in $\mathbb{C} P^{1} \times \mathbb{C} P^{1} \times \mathbb{C} P^{1}$, arises at the points from $\bar{F}_{12}^{1{ }^{\prime}{ }^{\prime}}$ and at the point $((1: 1),(1: 1),(1: 1))$. That is the reason for considering the blowup of $\mathcal{F}$ at the point $((1: 1),(1: 1),(1: 1))$ as an universal set of parameters.

Denote by $\bar{f}_{12,13}$ an extension of the homeomorphism $f_{12,13}: F_{12} \rightarrow F_{13}$ to $\mathcal{F} \backslash\left\{\bar{F}_{12}^{1^{\prime} 2^{\prime}} \cup((1:\right.$ $1),(1: 1),(1: 1))\}$.

We now prove the first statement of Theorem 9.1

Proposition 9.9. The map $\tilde{f}_{12,13}: \tilde{\mathcal{F}}_{12} \rightarrow \tilde{\mathcal{F}}_{13}$ defined by

$$
\begin{gathered}
\tilde{f}_{12,13}=\bar{f}_{12,13} \circ \pi \text { on } \tilde{\mathcal{F}}_{12} \backslash G_{12}, \\
\tilde{f}_{12,13}\left(\left((1: 0),(1: 0),\left(c_{3}: c_{3}^{\prime}\right)\right),(1: 1)\right)=\left(((1: 1),(1: 1),(1: 1)),\left(c_{3}: c_{3}^{\prime}\right)\right), \\
\tilde{f}_{12,13}\left(((1: 1),(1: 1),(1: 1)),\left(c: c^{\prime}\right)\right)=\left(\left((1: 0),(1: 0),\left(c: c^{\prime}\right)\right),(1: 1)\right),
\end{gathered}
$$

where $G_{12}=\left(((1: 1),(1: 1),(1: 1)),\left(c_{3}: c_{3}^{\prime}\right)\right) \cup\left(\left((1: 0),(1: 0),\left(c_{3}: c_{3}^{\prime}\right)\right),(1: 1)\right),\left(c_{3}: c_{3}^{\prime}\right) \in$ $\mathbb{C} P^{1}$ and $\pi: \tilde{\mathcal{F}} \rightarrow \mathcal{F}$ is a projection, is a homeomorphism.

Proof. It follows from Lemma 9.4 and Lemma 9.5 that the map $f_{12,13}$ continuously extends to a homeomorphism $f_{12,13}^{1^{\prime} 3}: \bar{F}_{12}^{1^{\prime} 3} \rightarrow \bar{F}_{13}^{11^{\prime}}$ defined by $f_{12,13}^{1^{\prime} 3}\left((1: 0),\left(c_{2}: c_{2}^{\prime}\right),(0: 1)\right)=\left((1: 1),\left(c_{2}:\right.\right.$ $\left.\left.c_{2}-c_{2}^{\prime}\right),\left(c_{2}: c_{2}-c_{2}^{\prime}\right)\right)$ and a homeomorphism $f_{12,13}^{2^{\prime} 3^{\prime}}: \bar{F}_{12}^{2^{\prime} 3^{\prime}} \rightarrow \bar{F}_{13}^{22^{\prime}}$ defined by $f_{12,13}^{2^{\prime} 3^{\prime}}\left(\left(c_{1}:\right.\right.$ $\left.\left.c_{1}^{\prime}\right),(1: 0),(1: 0)\right)=\left(\left(c_{1}: c_{1}-c_{1}^{\prime}\right),(1: 1),\left(c_{1}-c_{1}^{\prime}: c_{1}\right)\right)$. Together with Remark 7.7 this provides the proof for (26). In order to define an extension of $f_{12,13}$ on $\tilde{F}_{12}$ we need to define this extension on the set $\bar{F}_{12}^{1^{\prime} 2^{\prime}}$ and the divisor $\left.((1: 1),(1: 1),(1: 1)),\left(c: c^{\prime}\right)\right)$. The set $\bar{F}_{12}^{1^{\prime} 2^{\prime}}$ ca be embedded into $\tilde{F}_{12}$ as $\left(\left((1: 0),(1: 0),\left(c_{3}: c_{3}^{\prime}\right)\right),(1: 1)\right)$. Let us consider a sequence $y(n)=\left(\left(\left(1: c_{1}^{\prime}(n)\right),\left(1: c_{2}^{\prime}(n)\right),\left(c_{3}(n): c_{3}^{\prime}(n)\right)\right),\left(1-c_{1}^{\prime}(n): 1-c_{2}^{\prime}(n)\right)\right)$ from $\pi^{-1}\left(F_{12}\right) \subset \tilde{\mathcal{F}}_{12}$ which converges to a point $\left(\left((1: 0),(1: 0),\left(c_{3}: c_{3}^{\prime}\right)\right),(1: 1)\right)$. It holds $\tilde{f}_{12,13}\left(y_{n}\right)=f_{12,13}\left(\pi\left(y_{n}\right)\right)=\left(\left(1: 1-c_{1}^{\prime}(n)\right),\left(1: 1-c_{1}^{\prime}(n)\right),\left(\left(1-c_{1}^{\prime}(n)\right) c_{2}^{\prime}(n) c_{3}(n): c_{1}^{\prime}(n)\left(1-c_{2}^{\prime}(n)\right) c_{3}^{\prime}(n)\right)\right)$. 
The fourth coordinate of the point $\tilde{f}_{12,13}\left(y_{n}\right)$ in $\tilde{F}_{12,13}$ is $\left(c_{3}(n): c_{3}^{\prime}(n)\right)$. Namely, the fourth coordinate of this point is $\left(x_{1}(n): x_{2}(n)\right)$ such that that $c_{1}^{\prime}(n) x_{2}(n)=c_{2}^{\prime}(n) x_{1}(n)$, which implies that $\left(x_{1}(n): x_{2}(n)\right)=\left(1: \frac{c_{2}^{\prime}(n)}{c_{1}^{\prime}(n)}\right)$. The cubic equation implies that $\left(x_{1}(n): x_{2}(n)\right)=\left(c_{3}(n)\right.$ : $\left.c_{3}^{\prime}(n)\right)$. Since by Lemma 9.6 the continuous extension of $f_{12,13}$ on $\bar{F}_{12}^{2^{\prime} 3^{\prime}}$ maps $\bar{F}_{12}^{2^{\prime} 3^{\prime}}$ to $((1: 1),(1$ : $1),(1: 1))$ we obtain that the sequence $\tilde{f}_{12,13}\left(y_{n}\right) \subset \tilde{\mathcal{F}}_{13}$ converges to the point $(((1: 1),(1$ : $\left.1),(1: 1)),\left(c_{3}: c_{3}^{\prime}\right)\right)$. This proves the formula (27). The formula (28) follows in a similar way from (16) in Lemma7.5.

We prove the second statement of Theorem 9.1 , which states that a homeomorphic type of $\tilde{F}_{\sigma, i j}$ does not depend on a chart $M_{i j}$ :

Proposition 9.10. Let $W_{\sigma} \subset M_{i j}, M_{k l}$. Then $\tilde{f}_{i j, k l}\left(\tilde{F}_{\sigma, i j}\right)=\tilde{F}_{\sigma, k l}$ for the homeomorphism $\tilde{f}_{i j, k l}$ : $\tilde{\mathcal{F}}_{i j} \rightarrow \tilde{\mathcal{F}}_{k l}$.

Proof. The proof immediately follows: a sequence $z_{n}$ from the main stratum, which converges to a point from $W_{\sigma}$ defines, when considering it in the charts $M_{i j}$ and $M_{k l}$, the sequences of parameters $c_{n}^{i j}$ and $c_{n}^{k l}$. We have that $c_{n}^{k l}=f_{i j, k l}\left(c_{n}^{i j}\right)$, which implies that $\tilde{f}_{i j, k l}\left(\lim _{n \rightarrow \infty} c_{n}^{i j}\right)=\lim _{n \rightarrow \infty} c_{n}^{k l}$, which proves the statement.

In this way the proof of Theorem 9.1 is completed.

Now we prove Theorem 5.1 which states that any stratum in $G_{5,2}$ can be parametrized by considering the universal set of parameters just in one chart. Fix a chart $M_{i j}$ and consider an arbitrary stratum $W_{\sigma}$. Let $M_{k l}$ be an arbitrary chart such that $W_{\sigma} \subset M_{k l}$ and $\tilde{F}_{\sigma, k l}$ be the virtual space of parameters for $W_{\sigma}$ in the chart $M_{k l}$. Let

$$
\tilde{F}_{\sigma, i j, k l}=\tilde{f}_{k l, i j}\left(\tilde{F}_{\sigma, k l}\right) \subset \tilde{\mathcal{F}}_{i j},
$$

for the homeomorphism $\tilde{f}_{k l, i j}: \tilde{\mathcal{F}}_{k l} \rightarrow \tilde{\mathcal{F}}_{i j}$.

Proposition 9.11. It holds

$$
\tilde{F}_{\sigma, i j, k l}=\tilde{F}_{\sigma, i j, m n},
$$

for any charts $M_{k l}, M_{m n}$ such that $W_{\sigma} \subset M_{k l}, M_{m n}$.

Proof. Consider the homeomorphisms $\tilde{f}_{i j, k l}: \tilde{\mathcal{F}}_{i j} \rightarrow \tilde{\mathcal{F}}_{k l}, \tilde{f}_{i j, m n} ; \tilde{\mathcal{F}}_{i j} \rightarrow \tilde{\mathcal{F}}_{m n}$ and $\tilde{f}_{k l, m n}: \tilde{\mathcal{F}}_{k l} \rightarrow$ $\tilde{\mathcal{F}}_{m n}$. Clearly Lemma 8.2 holds after extension to blowup, that is $\tilde{f}_{k l, i j}=\tilde{f}_{m n, i j} \circ \tilde{f}_{k l, m n}$. By Proposition 9.10 we have that $\tilde{f}_{k l, m n}\left(\tilde{F}_{\sigma, k l}\right)=\tilde{F}_{\sigma, m n}$, which implies that $\tilde{f}_{k l, i j}\left(\tilde{F}_{\sigma, k l}\right)=\tilde{f}_{m n, i j}\left(\tilde{F}_{\sigma, m n}\right)$, that is $\tilde{F}_{\sigma, i j, k l}=\tilde{F}_{\sigma, i j, m n}$.

Remark 9.12. The set $\tilde{F}_{\sigma, i j, k l}$ we denote by $\tilde{F}_{\sigma, i j}$. Note that in this way we defined the sets $\tilde{F}_{\sigma, i j}$ for an arbitrary chart $M_{i j}$ and an arbitrary admissible set $\sigma$.

Proposition 9.11 can be reformulated as follows:

Proposition 9.13. Any stratum $W_{\sigma}$ in $G_{5,2}$ can be parametrized by $\tilde{F}_{\sigma, i j} \subset \tilde{\mathcal{F}}_{i j}$ such that for any two chart $M_{k l}, W_{\sigma} \subset M_{k l}$ it holds $\tilde{f}_{i j, k l}\left(\tilde{F}_{\sigma, i j}\right)=\tilde{F}_{\sigma, k l}$. 
9.1. The virtual spaces of parameters in the chart $M_{12}$. Using Theorem 9.1 we describe here the virtual spaces of parameters $\tilde{F}_{\sigma, 12} \subset \tilde{\mathcal{F}}_{12}$ for the strata $W_{\sigma}$ whose admissible polytopes are $K_{i j}(9), K_{i j, k l}, K_{i j}(7), P_{i j}, O_{i}$.

Theorem 9.14. The virtual space of parameters $\tilde{F}_{\sigma, 12}$ for a stratum $W_{\sigma}$ whose admissible polytope is $K_{i j}(9), K_{i j, k l}, K_{i j}(7), P_{i j}, O_{i}$ is as follows:

(1) $K_{12}(9) \rightarrow\left(((1: 1),(1: 1),(1: 1)),\left(c: c^{\prime}\right)\right), \quad K_{i j}(9) \rightarrow \tilde{F}_{i j, 12}, \quad i j \neq 12$;

(2) $K_{12,34} \rightarrow(((1: 1),(1: 1),(1: 1)),(0: 1)), \quad K_{12,35} \rightarrow(((1: 1),(1: 1),(1: 1)),(1: 0))$;

(3) $K_{12,45} \rightarrow(((1: 1),(1: 1),(1: 1)),(1: 1)), \quad K_{i j, k l} \rightarrow \tilde{F}_{(i j, k l), 12}, \quad i j \neq 12$;

(4) $K_{34}(7) \rightarrow\left((1: 1),\left(c: c^{\prime}\right),\left(c: c^{\prime}\right)\right), \quad K_{35}(7) \rightarrow\left(\left(c: c^{\prime}\right),(1: 1),\left(c^{\prime}: c\right)\right)$;

(5) $K_{45}(7) \rightarrow\left(\left(c: c^{\prime}\right),\left(c: c^{\prime}\right),(1: 1)\right), \quad K_{12}(7) \rightarrow\left(((1: 1),(1: 1),(1: 1)),\left(c: c^{\prime}\right)\right)$;

(6) $O_{1} \rightarrow F_{12} \cup \tilde{F}_{12,12} \cup \tilde{F}_{13,12} \cup \tilde{F}_{14,12} \cup \tilde{F}_{15,12}$;

(7) $O_{2} \rightarrow F_{12} \cup \tilde{F}_{12,12} \cup \tilde{F}_{23,12} \cup \tilde{F}_{24,12} \cup \tilde{F}_{25,12}$;

(8) $P_{34} \rightarrow\left((1: 1),\left(c: c^{\prime}\right),\left(c: c^{\prime}\right)\right), \quad P_{35}(7) \rightarrow\left(\left(c: c^{\prime}\right),(1: 1),\left(c^{\prime}: c\right)\right)$;

(9) $P_{45}(7) \rightarrow\left(\left(c: c^{\prime}\right),\left(c: c^{\prime}\right),(1: 1)\right), \quad P_{12}(7) \rightarrow\left(((1: 1),(1: 1),(1: 1)),\left(c: c^{\prime}\right)\right)$;

(10) $K_{i j}(7) \rightarrow \tilde{F}_{i j, 12}(7), \quad P_{i j} \rightarrow \tilde{F}_{i j, 12}(6), \quad i j \neq 34,34,45,12, \quad O_{i} \rightarrow \tilde{F}_{i, 12}, i \neq 1,2$.

where $\left(c: c^{\prime}\right) \in \mathbb{C} P^{1}$.

Proof. We demonstrate the proof for the stratum whose admissible polytope is $K_{12}(9)$. This stratum belongs to the chart $M_{13}$ and it can be directly checked that $\tilde{F}_{12,13}=\left((1: 0),(1: 0),\left(c_{3}: c_{3}^{\prime}\right)\right)$, $\left(c_{3}: c_{3}^{\prime}\right) \in \mathbb{C} P_{A}^{1}$. It follows from Proposition 9.9 that $\tilde{F}_{12,12}=\tilde{f}_{12,13}^{-1}\left(\tilde{F}_{12,13}\right)=(((1: 1),(1:$ $\left.1),(1: 1)),\left(c_{3}: c_{3}^{\prime}\right)\right)$, which proves the first statement. Note that for the strata with the admissible polytopes $\mathrm{O}_{1}$ and $\mathrm{O}_{2}$, the statement follows from Remark 6.6.

9.2. The projection from $\tilde{F}_{\sigma, i j}$ to $F_{\sigma}$. We prove that, using this construction, we can define a projection from $\tilde{F}_{\sigma, i j}$ to $F_{\sigma}$ for any non-orbit stratum $W_{\sigma}$ and any chart $M_{i j}$, .

Assume first that $F_{\sigma}$ is not a point. If $W_{\sigma} \subset M_{i j}$, from the construction of $\tilde{F}_{\sigma, i j}$ it follows that there is a canonical projection $\tilde{g}_{\sigma, i j}: \tilde{F}_{\sigma, i j} \rightarrow F_{\sigma, i j}$. If $W_{\sigma} \not \subset M_{i j}$, let $M_{k l}$ be a chart such that $W_{\sigma} \subset M_{k l}$ and let $\tilde{g}_{\sigma, k l}: \tilde{F}_{\sigma, k l} \rightarrow F_{\sigma, k l}$ be a canonical projection. Let $\tilde{f}_{i j, k l}: \tilde{F}_{\sigma, i j} \rightarrow \tilde{F}_{\sigma, k l}$ be the homeomorphism given by Theorem 9.1. We obtain a projection $\tilde{g}_{\sigma, i j, k l}: \tilde{F}_{\sigma, i j} \rightarrow F_{\sigma, k l}$ defined by the composition

$$
\tilde{F}_{\sigma, i j} \stackrel{\tilde{f}_{i j, k l}}{\rightarrow} \tilde{F}_{\sigma, k l} \stackrel{\tilde{g}_{\sigma, k l}}{\rightarrow} F_{\sigma, k l} .
$$

Let $f_{\sigma, k l, m n}: F_{\sigma, k l} \rightarrow F_{\sigma, m n}$ be the homeomorphism defined in 8.1 and 8.2 . From the construction of $\tilde{F}_{\sigma, i j}$ it directly follows:

Lemma 9.15. If $W_{\sigma} \subset M_{k l}, M_{m n}$ then

$$
f_{\sigma, k l, m n} \circ \tilde{g}_{\sigma, k l}=\tilde{g}_{\sigma, m n} \circ \tilde{f}_{k l, m n} .
$$

Thus, we obtain a projection $\tilde{g}_{\sigma, i j}: \tilde{F}_{\sigma, i j} \rightarrow F_{\sigma}$ defined by the map

$$
g_{\sigma, i j, k l}=f_{\sigma, k l} \circ \tilde{g}_{\sigma, i j, k l} \text {. }
$$

The projection $\tilde{g}_{\sigma, i j}$ does not depend on the choice of a chart $M_{k l}$, such that $W_{\sigma} \subset M_{k l}$.

Proposition 9.16. It holds $g_{\sigma, i j, k l}=g_{\sigma, i j, m n}$ for any $\sigma \in \mathcal{A}$ and any charts $M_{k l}, M_{m n}$, such that $W_{\sigma} \subset M_{k l}, M_{m n}$. 
Proof. Combining Lemma 8.2 and Lemma 9.15 we obtain the following commutative diagram:

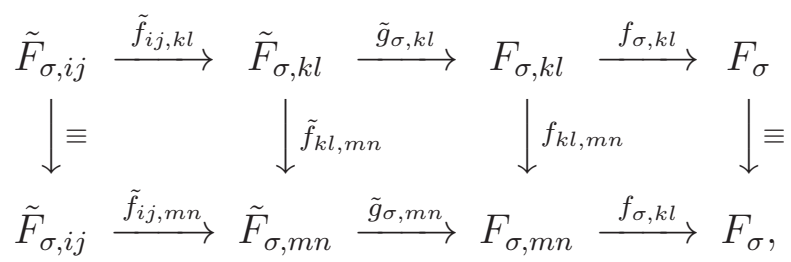

which proves the statement.

If $F_{\sigma}$ is a point then $g_{\sigma, i j}: \tilde{F}_{\sigma, i j} \rightarrow F_{\sigma}$ is obviously uniquely defined.

Altogether this leads:

Corollary 9.17. There exists a canonical projection $g_{\sigma, i j}: \tilde{F}_{\sigma, i j} \rightarrow F_{\sigma}$ for any $\sigma \in \mathcal{A}$ and any chart $M_{i j}$.

Remark 9.18. We pointed in Remark 4.19 that if $W_{\sigma} \subset M_{i j}$ and $F_{\sigma}$ is a point, one can not write down this point in the parameter coordinates for the chart $M_{i j}$. Thus, in this case $F_{\sigma, i j}$ is not defined, so it is not defined a projection $\tilde{F}_{\sigma, i j} \rightarrow F_{i j, \sigma}$. Nevertheless the projection $\tilde{F}_{\sigma, i j} \rightarrow F_{\sigma}$ is uniquely defined.

The space $\tilde{F}_{\sigma, i j}$ is in general larger then the space of parameters $F_{\sigma}$ as Proposition 6.3 shows.

\section{AN ALGEBRAiC MANIFOLd IN $\left(\mathbb{C} P^{1}\right)^{5}$ WHICH REALIZES $\tilde{\mathcal{F}}$}

We prove that the space of parameters $F$ of the main stratum, as well as its compactification $\tilde{\mathcal{F}}$ which we have described in previous sections can be realized by algebraic submanifolds in $\left(\mathbb{C} P^{1}\right)^{5}$.

Proposition 10.1. There is an embedding $I: F \rightarrow\left(\mathbb{C} P^{1}\right)^{5}$.

Proof. Let $L$ be a point from the main stratum $W$ of $G_{5,2}$ and $A_{L}$ an arbitrary $(5 \times 2)$ - matrix representing $L$. The rows of the matrix $L$ define the five non-collinear points in $\mathbb{C}^{2}$. We enumerate them by the rows which define them, that is $1,2,3,4,5$. We choose the following 5 lexicographically ordered variations of four of these five points: 1234, 1235, 1245, 1345, 2345 and map them to $\mathbb{C}^{*}$ by the cross-ratio from projective geometry. More precisely, we consider the map $\{1234,1235,1245,1345,2345\} \rightarrow \mathbb{C}^{*}$ defined by

$$
\begin{gathered}
1234 \rightarrow \frac{P^{13} P^{24}}{P^{14} P^{23}}, 1235 \rightarrow \frac{P^{13} P^{25}}{P^{15} P^{23}}, 1245 \rightarrow \frac{P^{14} P^{25}}{P^{15} P^{24}}, \\
1345 \rightarrow \frac{P^{14} P^{35}}{P^{15} P^{34}}, 2345 \rightarrow \frac{P^{24} P^{35}}{P^{25} P^{34}} .
\end{gathered}
$$

where $P^{i k}=P^{i k}\left(A_{L}\right)$ are the Plücker coordinates for $L$.

Using this map we obtain a map from the main stratum $W$ to $\left(\mathbb{C} P^{1}\right)^{5}$ defined by $i j k l \rightarrow$ $\left(P^{i k} P^{j l}: P^{i l} P^{j k}\right) \in \mathbb{C} P^{1}$. Note that this new map is well defined, it does not depend on the choice of a basis for $L$ neither on the choice of a basis in $\mathbb{C}^{n}$, since Plücker coordinates are defined uniquely up to common constant. This map is clearly equivariant for the action of the torus $\left(\mathbb{C}^{*}\right)^{5}$, since Plücker coordinates are equivariant for this action. In this we obtain an embedding of $F=W /\left(\mathbb{C}^{*}\right)^{5}$ in $\left(\mathbb{C} P^{1}\right)^{5}$.

Let $\left(c_{i}: c_{i}^{\prime}\right), 1 \leq i \leq 5$, be coordinates in $\left(\mathbb{C} P^{1}\right)^{5}$. We describe an algebraic manifold in $\left(\mathbb{C} P^{1}\right)^{5}$ which contains the image of $F$ by the above map $i j k l \rightarrow \frac{P^{i k} P^{j l}}{P^{i l} P^{j k}}$. 
Proposition 10.2. The image of an embedding $I: F \rightarrow\left(\mathbb{C} P^{1}\right)^{5}$ belongs to the intersection of the following hypers urfaces

$$
\begin{gathered}
c_{1} c_{2}^{\prime} c_{3}=c_{1}^{\prime} c_{2} c_{3}^{\prime}, \quad c_{2}^{\prime} c_{4}\left(c_{1}-c_{1}^{\prime}\right)=c_{1}^{\prime} c_{4}^{\prime}\left(c_{2}-c_{2}^{\prime}\right), \\
\left(c_{1}-c_{1}^{\prime}\right) c_{2} c_{5}=c_{1}\left(c_{2}-c_{2}^{\prime}\right) c_{5}^{\prime}, \quad c_{3} c_{4}^{\prime} c_{5}=c_{3}^{\prime} c_{4} c_{5}^{\prime} .
\end{gathered}
$$

Proof. It immediately follows from (31) that the first equation in (33) is satisfied. We now use this conclusion and properties of the cross-ratio. Since

$$
1324 \rightarrow 1-\frac{c_{1}}{c_{1}^{\prime}}, \quad 1325 \rightarrow 1-\frac{c_{2}}{c_{2}^{\prime}}, \quad 1345 \rightarrow \frac{c_{4}}{c_{4}^{\prime}},
$$

we analogously obtain that

$$
\left(c_{1}^{\prime}-c_{1}\right) c_{2}^{\prime} c_{4}=c_{1}^{\prime}\left(c_{2}^{\prime}-c_{2}\right) c_{4}^{\prime}
$$

Since

we conclude that

$$
2314 \rightarrow 1-\frac{c_{1}^{\prime}}{c_{1}}, \quad 2315 \rightarrow 1-\frac{c_{2}^{\prime}}{c_{2}}, \quad 2345 \rightarrow \frac{c_{5}}{c_{5}^{\prime}},
$$

Since

$$
\left(c_{1}-c_{1}^{\prime}\right) c_{2} c_{5}=c_{1}\left(c_{2}-c_{2}^{\prime}\right) c_{5}^{\prime}
$$

it follows that

$$
4512 \rightarrow \frac{c_{3}^{\prime}}{c_{3}}, \quad 4513 \rightarrow \frac{c_{4}}{c_{4}^{\prime}}, \quad 4523 \rightarrow \frac{c_{5}}{c_{5}^{\prime}},
$$

$$
c_{3} c_{4}^{\prime} c_{5}=c_{3}^{\prime} c_{4} c_{5}^{\prime}
$$

Denote by $\mathcal{G}$ an algebraic manifold in $\left(\mathbb{C} P^{1}\right)^{5}$ obtained as the intersection of the surfaces given by Proposition 10.2 .

Lemma 10.3. The virtual spaces of parameters $\tilde{F}_{i j, 12} \cong \mathbb{C} P_{A}^{1}$ of the strata whose admissible polytopes are $K_{i j}(9)$ for $T^{5}$-action on $G_{5,2}$, can be embedded into $\mathcal{G}$ as follows:

(1) $\tilde{F}_{13,12} \rightarrow\left((0: 1),(0: 1),\left(c: c^{\prime}\right),(1: 1),\left(c^{\prime}: c\right)\right)$,

(2) $\tilde{F}_{14,12} \rightarrow\left((1: 0),\left(c: c^{\prime}\right),(0: 1),(0: 1),\left(c-c^{\prime}: c\right)\right)$,

(3) $\tilde{F}_{15,12} \rightarrow\left(\left(c: c^{\prime}\right),(1: 0),(1: 0),(1: 0),\left(c: c-c^{\prime}\right)\right)$,

(4) $\tilde{F}_{23,12} \rightarrow\left((1: 0),(1: 0),\left(c: c^{\prime}\right),\left(c: c^{\prime}\right),(1: 1)\right)$,

(5) $\tilde{F}_{24,12} \rightarrow\left((0: 1),\left(c: c^{\prime}\right),(1: 0),\left(c^{\prime}-c: c^{\prime}\right),(0: 1)\right)$,

(6) $\tilde{F}_{25,12} \rightarrow\left(\left(c: c^{\prime}\right),(0: 1),(0: 1),\left(c^{\prime}: c^{\prime}-c\right),(1: 0)\right)$,

(7) $\tilde{F}_{34,12} \rightarrow\left((1: 1),\left(c: c^{\prime}\right),\left(c: c^{\prime}\right),(1: 0),(1: 0)\right)$,

(8) $\tilde{F}_{35,12} \rightarrow\left(\left(c: c^{\prime}\right),(1: 1),\left(c^{\prime}: c\right),(0: 1),(0: 1)\right)$,

(9) $\tilde{F}_{12,12} \rightarrow\left((1: 1),(1: 1),(1: 1),\left(c: c^{\prime}\right),\left(c: c^{\prime}\right)\right)$,

(10) $\tilde{F}_{45,12} \rightarrow\left(\left(c: c^{\prime}\right),\left(c: c^{\prime}\right),(1: 1),(1: 1),(1: 1)\right)$.

Proof. The stratum $W_{i j}$ whose admissible polytope is $K_{i j}(9)$ is defined by the condition that $P^{i j}=$ 0 . Combining this and (31), (32) we immediately obtain the embeddings $(1)-(8)$. In order to prove that the embeddings (9) and (10) hold we use in addition the Plücker relations presented in Example 2.3.

In the same way we prove the following lemmas: 
Lemma 10.4. The virtual spaces of parameters $\tilde{F}_{(i j, k l), 12} \cong\{*\}$ of the strata whose admissible polytopes are $K_{i j, k l}$ can be embedded into $\mathcal{G}$ as follows

- $\tilde{F}_{(14,23), 12} \rightarrow((1: 0),(1: 0),(0: 1),(0: 1),(1: 1))$,

- $\tilde{F}_{(13,24), 12} \rightarrow((0: 1),(0: 1),(1: 0),(1: 1),(0: 1))$,

- $\tilde{F}_{(15,24), 12} \rightarrow((0: 1),(1: 0),(1: 0),(1: 0),(0: 1))$,

- $\tilde{F}_{(23,45) .12} \rightarrow((1: 0),(1: 0),(1: 1),(1: 1),(1: 1))$,

- $\tilde{F}_{(24,35), 12} \rightarrow((0: 1),(1: 1),(1: 0),(0: 1),(0: 1))$,

- $\tilde{F}_{(25,34), 12} \rightarrow((1: 1),(0: 1),(0: 1),(1: 0),(1: 0))$,

- $\tilde{F}_{(15,23), 12} \rightarrow((1: 0),(1: 0),(1: 0),(1: 0),(1: 1))$,

- $\tilde{F}_{(13,25), 12} \rightarrow((0: 1),(0: 1),(0: 1),(1: 1),(1: 0))$,

- $\tilde{F}_{(14,25), 12} \rightarrow((1: 0),(0: 1),(0: 1),(0: 1),(1: 0))$,

- $\tilde{F}_{(13,45), 12} \rightarrow((0: 1),(0: 1),(1: 1),(1: 1),(1: 1))$,

- $\tilde{F}_{(14,35), 12} \rightarrow((1: 0),(1: 1),(0: 1),(0: 1),(0: 1))$,

- $\tilde{F}_{(15,34), 12} \rightarrow((1: 1),(1: 0),(1: 0),(1: 0),(1: 0))$.

- $\tilde{F}_{(12,34), 12} \rightarrow((1: 1),(1: 1),(1: 1),(1: 0),(1: 0))$,

- $\tilde{F}_{(12,35), 12} \rightarrow((1: 1),(1: 1),(1: 1),(0: 1),(0: 1))$,

- $\tilde{F}_{(12,45), 12} \rightarrow((1: 1),(1: 1),(1: 1),(1: 1),(1: 1))$.

Lemma 10.5. The virtual spaces of parameters $\tilde{F}_{i j, 12}(7) \cong \mathbb{C} P^{1}$ of the strata whose admissible polytopes are $K_{i j}(7)$ can be embedded into $\mathcal{G}$ as follows

- $\tilde{F}_{23,12}(7) \rightarrow\left((1: 0),(1: 0),\left(c: c^{\prime}\right),\left(c: c^{\prime}\right),(1: 1)\right)$,

- $\tilde{F}_{24,12}(7) \rightarrow\left((0: 1),\left(c: c^{\prime}\right),(1: 0),\left(c^{\prime}-c: c^{\prime}\right),(0: 1)\right)$,

- $\tilde{F}_{25,12}(7) \rightarrow\left(\left(c: c^{\prime}\right),(0: 1),(0: 1),\left(c: c^{\prime}-c\right),(1: 0)\right)$,

- $\tilde{F}_{13,12}(7) \rightarrow\left((0: 1),(0: 1),\left(c: c^{\prime}\right),(1: 1),\left(c^{\prime}: c\right)\right)$,

- $\tilde{F}_{14,12}(7) \rightarrow\left((1: 0),\left(c: c^{\prime}\right),(0: 1),(0: 1),\left(c-c^{\prime}: c\right)\right)$,

- $\tilde{F}_{15,12}(7) \rightarrow\left(\left(c: c^{\prime}\right),(1: 0),(1: 0),(1: 0),\left(c: c-c^{\prime}\right)\right)$,

- $\tilde{F}_{12,12}(7) \rightarrow\left((1: 1),(1: 1),(1: 1),\left(c: c^{\prime}\right),\left(c: c^{\prime}\right)\right)$,

- $\tilde{F}_{34,12}(7) \rightarrow\left((1: 1),\left(c: c^{\prime}\right),\left(c: c^{\prime}\right),(1: 0),(1: 0)\right)$,

- $\tilde{F}_{35,12}(7) \rightarrow\left(\left(c: c^{\prime}\right),(1: 1),\left(c^{\prime}: c\right),(0: 1),(0: 1)\right)$,

- $\tilde{F}_{45,12}(7) \rightarrow\left(\left(c: c^{\prime}\right),\left(c: c^{\prime}\right),(1: 1),(1: 1),(1: 1)\right)$.

Lemma 10.6. The virtual spaces of parameters $\tilde{F}_{i, 12} \cong\left\{\left(c_{1}: c_{1}^{\prime}\right),\left(c_{2}: c_{2}^{\prime}\right),\left(c_{3}: c_{3}^{\prime}\right)\right) \mid c_{1} c_{2}^{\prime} c_{3}=$ $\left.c_{1}^{\prime} c_{2} c_{3}^{\prime}, c_{3}, c_{3}^{\prime} \neq 0, c_{3} \neq c_{3}^{\prime}\right\}$ of the strata whose admissible polytopes are $O_{i}$ can be embedded into $\mathcal{G}$.

Proof. The virtual space of parameters for $O_{i}$ is given by $\tilde{F}_{i, 12}=F \cup\left(\cup_{j \neq i} \tilde{F}_{i j, 12}\right)$. An embedding of $\tilde{F}_{i}$ in $\mathcal{G}$ is given by (31), (32) and Lemma 10.3.

In an analogous way we verify an existence of the embedding of virtual spaces of parameters for all other strata. Since the virtual spaces of parameters $\tilde{F}_{\sigma, 12}$ produce a compactification of $F$ that coincides with $\tilde{\mathcal{F}}$, previous lemmas together with Proposition 10.1 and Proposition 10.2 imply:

Theorem 10.7. The embedding $I: F \rightarrow\left(\mathbb{C} P^{1}\right)^{5}$ extends to an embedding $\hat{I}: \tilde{\mathcal{F}} \rightarrow\left(\mathbb{C} P^{1}\right)^{5}$, such that $\hat{I}(\tilde{\mathcal{F}}) \subset \mathcal{G}$. 
Since $\tilde{\mathcal{F}}$ and $\mathcal{G}$ are compact manifolds of the same dimension we deduce:

Corollary 10.8. The universal space of parameters $\tilde{\mathcal{F}}$ is homeomorphic to the algebraic manifold $\mathcal{G} \subset\left(\mathbb{C} P^{1}\right)^{5}$.

\section{THE ORBIT SPACE $G_{5,2} / T^{5}$}

11.1. Summary. We summarize results from previous sections about $F_{\sigma, i j}$ and $\tilde{F}_{\sigma, i j}$ for all admissible polytopes $P_{\sigma}$.

(1) $P_{\sigma}=\Delta_{5,2}$ then $F_{\sigma} \cong \tilde{F}_{\sigma, i j} \cong F$,

(2) $P_{\sigma}=K_{p q}(9)$ then $F_{\sigma} \cong \tilde{F}_{\sigma, i j} \cong \mathbb{C} P_{A}^{1}$,

(3) $P_{\sigma}=O_{l}$ then $F_{\sigma} \cong \mathbb{C} P_{A}^{1}$ and $\tilde{F}_{\sigma, i j} \cong\left(\mathbb{C} P_{A}^{1} \times \mathbb{C} P^{1} \times \mathbb{C} P^{1}\right) \cap \tilde{\mathcal{F}}$,

(4) $P_{\sigma}=K_{k l, m n}$ then $F_{\sigma}=\tilde{F}_{\sigma, i j}$ is a point,

(5) $P_{\sigma} \neq \Delta_{5,2}, K_{p q}(9), O_{l}, K_{k l, m n}$ then $F_{\sigma}$ is a point and $\tilde{F}_{\sigma, i j}$ is not a point.

11.2. Description of the orbit space $G_{5,2} / T^{5}$. Let $\mathcal{P}$ be the formal union of all admissible polytopes:

$$
\mathcal{P}=\cup_{\sigma \in \mathcal{A}} P_{\sigma} .
$$

Denote by $\tilde{p}: \mathcal{P} \rightarrow \Delta_{5,2}$ the canonical projection. There is a canonical map $\tilde{\mu}: G_{5,2} / T^{5} \rightarrow \mathcal{P}$ defined by

$$
\tilde{\mu}(X)=x \in P_{\sigma} \text { if and only if } X \in W_{\sigma} / T^{5} \text { and } \tilde{p}(x)=\hat{\mu}(X) .
$$

In other words $\tilde{\mu}$ is defined by

$$
\tilde{p} \circ \tilde{\mu}=\hat{\mu} \text {. }
$$

We assume $\mathcal{P}$ to be equipped with the topology induced by the map $\tilde{\mu}: U \subset \mathcal{P}$ is an open set if and only if $\tilde{\mu}^{-1}(U)$ is an open in $G_{5,2} / T^{5}$. Note that $\mathcal{P}$ is a compact space for this topology.

Let us consider the set

$$
\mathcal{E}=\cup_{\sigma \in \mathcal{A}} \stackrel{\circ}{P}_{\sigma} \times \widetilde{F}_{\sigma, i j}
$$

The embeddings $\stackrel{\circ}{P}_{\sigma} \hookrightarrow \mathcal{P}$ and $\widetilde{F}_{\sigma, i j} \hookrightarrow \tilde{\mathcal{F}}$ define a canonical map $f: \mathcal{E} \rightarrow \mathcal{P} \times \widehat{\mathcal{F}}$. It follows from the definition of $\tilde{\mathcal{F}}$ and the description of admissible polytopes that the map $f$ is a surjection. We assume that $\mathcal{E}$ is equipped with the topology induced by the map $f$, that is $U \subset \mathcal{E}$ is an open set if and only if $f(U)$ is an open set in $\mathcal{P} \times \tilde{\mathcal{F}}$. The space $\mathcal{E}$ is a compact space for this topology. By Theorem 9.1, considering all charts for $G_{5,2}$, we obtain in this way ten homeomorphic topological spaces .

Recall that $G_{5,2} / T^{5}=\cup_{\sigma \in \mathcal{A}} W_{\sigma} / T^{\sigma}$ and define a mapping

$$
H: \mathcal{E} \rightarrow G_{5,2} / T^{5}, \quad H\left(\stackrel{\circ}{P}_{\sigma} \times \widetilde{F}_{\sigma, i j}\right) \subset W_{\sigma} / T^{\sigma}
$$

by

$$
H(x, c)=h_{\sigma}^{-1}\left(x, g_{\sigma, i j}(c)\right)
$$

Here $h_{\sigma}: W_{\sigma} / T^{\sigma} \rightarrow \stackrel{\circ}{P}_{\sigma} \times F_{\sigma}$ is a canonical homeomorphism stated by Proposition 3.7 and $g_{\sigma, i j}: \widetilde{F}_{\sigma, i j} \rightarrow F_{\sigma}$ is a canonical projection stated by Corollary 9.17 .

Theorem 11.1. The orbit space $G_{5,2} / T^{5}$ is a quotient space of the space $\mathcal{E}$ by an equivalence relation defined by the map $H$. 
Proof. We prove that the map $H$ is continuous and that it is a surjection. Since the space $G_{5,2}$ is Hausdorff, it will imply that the quotient space of $\mathcal{E}$ by the map $H$ and the orbit space $G_{5,2} / T^{5}$ are homeomorphic. The fact that the map $H$ is continuous follows from the construction of the space $\mathcal{E}$. We verify it in a couple of illustrative cases.

Assume that we fixed the $M_{12}$ and it is given a sequence $\left(x_{n}, c_{n}\right) \in \stackrel{\circ}{\Delta}_{5,2} \times \tilde{F}_{12}$, which converges to a point $\left(x_{0}, c_{0}\right) \in \stackrel{\circ}{K}_{13} \times \tilde{F}_{13,12}$. Since $c_{n}=\left(\left(c_{1 n}: c_{1 n}^{\prime}\right),\left(c_{2 n}: c_{2 n}^{\prime}\right),\left(c_{3 n}: c_{3 n}^{\prime}\right)\right)$ and $c_{0}=((1$ : $\left.0),(1: 0),\left(c: c^{\prime}\right)\right)$, we obtain that $c_{1 n}^{\prime}, c_{2 n}^{\prime} \rightarrow 0$. Let $p_{n}=h^{-1}\left(x_{n}, c_{n}\right) \in W / T^{5}$, then $p_{n}$ writes in the chart $M_{12}$ as $p_{n}=\left[\left(z_{1 n}, \ldots, z_{6 n}\right)\right]$ and it holds $c_{1 n}^{\prime} z_{1 n} z_{5 n}=c_{1 n} z_{2 n} z_{4 n}, c_{2 n}^{\prime} z_{1 n} z_{6 n}=c_{2 n} z_{3 n} z_{4 n}$, $c_{3 n}^{\prime} z_{2 n} z_{6 n}=c_{3 n} z_{3 n} z_{4 n}$. The conditions that $c_{1 n}^{\prime}, c_{2 n}^{\prime} \rightarrow 0$ and that $\hat{\mu}\left(p_{n}\right)=x_{n} \rightarrow x_{0} \in \stackrel{\circ}{K}{ }_{13}$ implies that $z_{4 n} \rightarrow 0$. Let us consider a sequence $q_{n}$ in $G_{5,2} / T^{5}$ which is given in the chart $M_{12}$ by $q_{n}=\left[\left(z_{1 n}, z_{2 n}, z_{3 n}, 0, z_{5 n}, z_{6 n}\right)\right]$. Note that apart from the Plücker coordinate $P^{13}$ all other Plücker coordinates for $p_{n}$ and $q_{n}$ coincide and $P^{13}\left(p_{n}\right) \rightarrow 0=P^{13}\left(q_{n}\right)$. It implies that $\lim \hat{\mu}\left(q_{n}\right)=$ $\lim \hat{\mu}\left(p_{n}\right)=\lim x_{n}=x_{0}$. The sequence $q_{n}$ belongs to the stratum whose admissible polytope is $K_{13}$ and $h_{13,12}\left(q_{n}\right)=\left(y_{n},\left(c_{3 n}: c_{3 n}^{\prime}\right)\right)$, where $y_{n}=\hat{\mu}\left(q_{n}\right)$. Since $h_{13,12}: W_{13} / T^{5} \rightarrow \stackrel{\circ}{K}{ }_{13} \times \mathbb{C} P_{A}^{1}$ is a homeomorphism and $\left(y_{n},\left(c_{3 n}: c_{3 n}^{\prime}\right)\right) \rightarrow\left(x_{0},\left(c: c^{\prime}\right)\right) \in \stackrel{\circ}{K}{ }_{13} \times \mathbb{C} P_{A}^{1}$, it implies that $q_{n} \rightarrow p_{0}=$ $h_{13,12}^{-1}\left(x_{0},\left(c: c^{\prime}\right)\right)$. Then the sequence $p_{n}$ is convergent as well and $p_{n} \rightarrow h_{13,12}^{-1}\left(x_{0}, f_{13,12}\left(c_{0}\right)\right)=$ $H\left(x_{0}, c_{0}\right)$.

Let us consider now a sequence $\left(x_{n}, c_{n}\right) \in \stackrel{\circ}{\Delta}_{5,2} \times \tilde{F}_{12}$ converging to a point $\left(x_{0}, c_{0}\right) \in \stackrel{\circ}{K} 13$ $(7) \times \tilde{F}_{13,12}(7)$. Then $c_{0}=\left((1: 0),(1: 0),\left(c: c^{\prime}\right)\right)$ and, as previously, it holds $c_{1 n}^{\prime}, c_{2 n}^{\prime} \rightarrow$ 0 . Now, if we consider the sequence $p_{n}=\left[\left(z_{1 n}, \ldots, z_{6 n}\right)\right]=h\left(x_{n}, c_{n}\right)$ in the chart $M_{12}$, the condition that $\hat{\mu}\left(p_{n}\right)=x_{n} \rightarrow x_{0} \in \stackrel{\circ}{K} 13$ (7) implies that $z_{2 n}, z_{3 n} \rightarrow 0$. The sequence $q_{n}=$ $\left[\left(z_{1 n}, 0,0, z_{4 n}, z_{5 n}, z_{6 n}\right)\right]$ belongs to the stratum whose admissible polytope is $K_{13}(7)$ and $\lim \hat{\mu}\left(q_{n}\right)=$ $\lim \hat{\mu}\left(p_{n}\right)=\lim x_{n}=x_{0}$. Since the space of parameters $F_{13}(7)$ is a point, it follows that $\tilde{\mu}: W_{13}(7) / T^{5} \rightarrow \stackrel{\circ}{K}_{13}(7)$ is a homeomorphism. Therefore, as $\tilde{\mu}\left(q_{n}\right)=y_{n} \rightarrow x_{0} \in \stackrel{\circ}{K_{13}}(7)$ we conclude that $\tilde{\mu}^{-1}\left(y_{n}\right) \rightarrow \tilde{\mu}^{-1}\left(x_{0}\right)=p_{0}$. It implies that the sequence $p_{n}$ is convergent and $p_{n} \rightarrow p_{0}=H\left(x_{0}, c_{0}\right)$.

Let us consider now a sequence $\left(x_{n}, c_{n}\right) \in \stackrel{\circ}{\Delta}_{5,2} \times \tilde{F}_{12}$ converging to a point $\left(x_{0}, c_{0}\right) \in \stackrel{\circ}{O}_{3}$ $\times \tilde{F}_{3,12}$. Then $c_{0}=\left(\left(c_{1}: c_{1}^{\prime}\right),\left(c_{2}: c_{2}^{\prime}\right),\left(c_{3}: c_{3}^{\prime}\right)\right)$, where $\left(c_{i}: c_{i}^{\prime}\right) \in \mathbb{C} P^{1}, i=1,2$, and $\left(c_{3}: c_{3}^{\prime}\right) \in \mathbb{C} P_{A}^{1}$. Taking $p_{n}=\left[\left(z_{1 n}, \ldots, z_{6 n}\right)\right]=h\left(x_{n}, c_{n}\right)$ in the chart $M_{12}$, the condition that $\hat{\mu}\left(p_{n}\right)=x_{n} \rightarrow x_{0} \in \stackrel{\circ}{O}_{3}$ and that $O_{3}$ is a facet of $\Delta_{5,2}$ implies $z_{1 n}, z_{4 n} \rightarrow 0$. The sequence $q_{n}=\left[\left(0, z_{2 n}, z_{3 n}, 0, z_{5 n}, z_{6 n}\right)\right]$ belongs to the stratum whose admissible polytope is $O_{3}$ and $\lim \hat{\mu}\left(q_{n}\right)=\lim \hat{\mu}\left(p_{n}\right)=\lim x_{n}=x_{0}$. Further, $h_{3,12}\left(q_{n}\right)=\left(y_{n},\left(c_{3 n}: c_{3 n}^{\prime}\right)\right)$, where $y_{n}=\hat{\mu}\left(q_{n}\right)$. Since $\left(y_{n},\left(c_{3 n}: c_{3 n}^{\prime}\right)\right) \rightarrow\left(x_{0},\left(c_{3}: c_{3}^{\prime}\right)\right)$, we conclude that $q_{n} \rightarrow h_{3,12}^{-1}\left(x_{0},\left(c_{3}: c_{3}^{\prime}\right)\right)=p_{0}$. Therefore, $p_{n} \rightarrow p_{0}=h_{3,12}^{-1}\left(x_{0}, f_{3,12}\left(c_{0}\right)\right)=H\left(x_{0}, c_{0}\right)$.

Thus, $H$ is continuous. Since $G_{5,2} / T^{5}=\cup_{\sigma \in \mathcal{A}} W_{\sigma} / T^{5}$ and $H\left(\stackrel{\circ}{P}_{\sigma} \times \tilde{F}_{\sigma, i j}\right)=W_{\sigma} / T^{5}$, the map $H$ is onto, which implies that it induces a homeomorphism between the quotient space of $\mathcal{E}$ defined by $H$ and the orbit space $G_{5,2} / T^{5}$.

Corollary 11.2. The equivalence relation on $\mathcal{E}$ defined by the map $H$ is given by the equivalence relation on each $\stackrel{\circ}{P}_{\sigma} \times \tilde{F}_{\sigma, i j}$ :

$$
\left(x_{1}, c_{1}\right) \backsim\left(x_{2}, c_{2}\right) \text { if and only if } x_{1}=x_{2}, g_{\sigma, i j}\left(c_{1}\right)=g_{\sigma, i j}\left(c_{2}\right) \text {. }
$$


Note that the equivalence relation $\simeq$ is trivial for $P_{\sigma}=\Delta_{5,2}, K_{i j}(9), K_{i j, k l}$ since $\tilde{F}_{\sigma, i j} \cong F_{\sigma}$. This relation is non-trivial for the octahedra $O_{i}$, pyramids $K_{i j}(7)$ and prisms $P_{i j}$.

Corollary 11.3. The orbit space $G_{5.2} / T^{5}$ is homeomorphic to $\mathcal{E} / \approx$, where (38)

$\left(x_{\sigma_{1}}, c_{\sigma_{1}, i j}\right) \backsim\left(x_{\sigma_{2}}, c_{\sigma_{2}, i j}\right)$ if and only if $\sigma_{1}=\sigma_{2}, \quad x_{\sigma_{1}}=x_{\sigma_{2}}$ and $g_{\sigma_{1}, i j}\left(c_{\sigma_{1}, i j}\right)=g_{\sigma_{2}, i j}\left(c_{\sigma_{2}, i j}\right)$.

Remark 11.4. The formula (38) is explicit. Namely, while $F_{\sigma}$ are abstract spaces of parameters of the strata, the spaces $\tilde{F}_{\sigma, i j}$ are explicit subspaces of $\tilde{\mathcal{F}}$ which makes this formula applicable. Theorem 9.14, Proposition 6.1, Proposition 6.2 and Proposition 6.3 provide the description of $\tilde{F}_{\sigma, 12}$ when $P_{\sigma}$ is an admissible polytope which belongs to the interior of $\Delta_{n, k}$.

Let us consider now the projection $\mathcal{P} \times \tilde{\mathcal{F}} \rightarrow \Delta_{5,2} \times \tilde{\mathcal{F}}$. Define an equivalence relation on $\Delta_{5,2} \times \tilde{\mathcal{F}}$ by

$$
\left(x, c_{1, i j}\right) \backsim\left(x, c_{2, i j}\right) \text { if and only if } c_{1, i j}, c_{2, i j} \in \tilde{F}_{\sigma, i j}, x \in \stackrel{\circ}{P_{\sigma}} \text { and } g_{\sigma, i j}\left(c_{1, i j}\right)=g_{\sigma, i j}\left(c_{2, i j}\right) .
$$

It follows from (38) and (39) that there is a canonical projection from the space $(\mathcal{P} \times \tilde{\mathcal{F}}) / \backsim$ to the space $\left(\Delta_{5,2} \times \tilde{\mathcal{F}}\right) / \simeq$. Corollary 11.3 implies

Corollary 11.5. There is a canonical continuous map $G_{5,2} / T^{5} \rightarrow\left(\Delta_{5,2} \times \tilde{\mathcal{F}}\right) / \backsim$, where $\backsim$ is an equivalence relation defined by (39).

Example 11.6. We demonstrate the application of the formula (38) in the case of Grassmann manifold $G_{4,2}$ whose orbit space is described in [7]. In this case, as it follows from [7], the admissible polytopes are the octahedron $\Delta_{4,2}$, the six pyramids $P_{i}, 1 \leq i \leq 6$, the three rectangles $R_{12}, R_{34}$, $R_{56}$ and the faces of $\Delta_{4,2}$. We assume the numeration to be such that $P_{1} \cap P_{2}=R_{12}, P_{3} \cap P_{4}=R_{34}$ and $P_{5} \cap P_{6}=R_{56}$. It is proved in [7] that $\tilde{F}_{12}=F_{12}=\mathbb{C} \backslash\{0,1\}$. Denote by $\tilde{F}_{12}\left(P_{\sigma}\right)$ the virtual space of parameters of an admissible polytope $P_{\sigma}$ in the chart $M_{12}$. It follows from [7] that $\tilde{F}_{12}\left(P_{1}\right)=\tilde{F}_{12}\left(P_{2}\right)=0, \tilde{F}_{12}\left(P_{3}\right)=\tilde{F}_{12}\left(P_{4}\right)=1, \tilde{F}_{12}\left(P_{5}\right)=\tilde{F}_{12}\left(P_{6}\right)=\infty$, then $\tilde{F}_{12}\left(R_{12}\right)=0$, $\tilde{F}_{12}\left(R_{34}\right)=1$ and $\tilde{F}_{12}\left(R_{56}\right)=\infty$, while $\tilde{F}_{12}\left(P_{I}\right)=\mathbb{C} P^{1}$ for the faces of $\Delta_{4,2}$. Then formula (38) gives

$$
G_{4,2} / T^{4} \cong\left(\cup_{I} \stackrel{\circ}{P}_{I} \times \tilde{F}_{i j}\left(P_{I}\right)\right) / \bumpeq,
$$

where $\left(x_{I}, c_{I}\right) \backsim\left(x_{J}, c_{J}\right)$ if and only if $I=J$ and $x_{I}=x_{J} \in \partial \Delta_{4,2}$. This can be further written as

$$
G_{4,2} \cdot T^{4} \cong\left(\Delta_{4,2} \times \mathbb{C} P^{1}\right) / \simeq,
$$

where $(x, c) \backsim\left(x^{\prime}, c^{\prime}\right)$ if and only if $x=x^{\prime} \in \partial \Delta_{4,2}$, which is exactly the formula obtained in [7].

Remark 11.7. Let us consider in $\mathbb{C}^{5}$ coordinates subspaces of the dimensions $k=2,3,4$ and the corresponding embeddings of $G_{k, 2}$ in $G_{5,2}$. Since an embedding of a coordinate subspace is equivariant for the coordinate vise action of the torus $T^{5}$ we obtain an equivariant embedding of $G_{k, 2}$ in $G_{5,2}$. In this way the embeddings of the corresponding orbit spaces are defined as well. For $k=4$ we obtain the embeddings of the five 5 -dimensional spheres in $G_{5,2} / T^{5}$. Moreover, we know how these spheres are glued together in $G_{5,2} / T^{2}$. Any pair of these 4-dimensional coordinate subspaces intersect each other in $\mathbb{C}^{5}$ along a coordinate subspace $\mathbb{C}^{3}$. A coordinate subspace $\mathbb{C}^{3}$ produce in $G_{5,2}$ the complex projective space $\mathbb{C} P^{2}=G_{3,2}$ whose orbit space, by the torus action, is the 2-dimensional simplex. In this way, using the cell decomposition of $G_{4,2} / T^{4}$ described in [7], we obtain that the orbit spaces $G_{4,2} / T^{4}$ are glued in $G_{5,2} / T^{2}$ along the 2-dimensional simplices. 


\section{The Homology GROUPS OF $G_{5,2} / T^{5}$}

We compute the homology groups of the orbit space $G_{5,2} / T^{5}$ appealing to Theorem 11.1 and results on the description of the orbit spaces of the strata, their admissible polytopes, spaces of parameters and virtual spaces of parameters.

We first compute the top degree homology group for $G_{n, 2} / T^{n}$ for $n \geq 4$. Let $V_{2}=\tilde{\mu}^{-1}\left(\cup P_{\sigma}\right)$, where $P_{\sigma}$ runs through all admissible polytopes different from $\Delta_{5,2}$. Then $V_{2}$ is a closed subset in $G_{n, 2} / T^{n}$ and $\left(G_{5,2} / T^{n}\right) \backslash V_{2}=W / T^{n}$ is the orbit space of the main stratum. Since $W / T^{n}$ is a dense set in $G_{5,2} / T^{5}$, it follows that $\left(G_{n, 2} / T^{n}\right) / V_{2}$ is the Alexandrov one-point compactification of $W / T^{n}$. Recall that $W / T^{n} \cong \stackrel{\circ}{\Delta}_{n, 2} \times F$ and denote its one-point compactification by $\left(\stackrel{\circ}{\Delta}_{n, 2} \times F\right)^{\star}$. Note that $F$ is homeomorphic to $\mathbb{C} P_{A}^{1} \times \mathbb{C} P_{A}^{1}$, which implies that $\Delta_{5,2} \times\left(\mathbb{C} P^{1} \times \mathbb{C} P^{1}\right)$ is a compactification of $\stackrel{\circ}{\Delta}_{n, 2} \times F$. Proposition 2.19 implies that

$$
\left(\stackrel{\circ}{\Delta}, 2_{n, 2} \times F_{n}\right)^{\star} \cong\left(\Delta_{5,2} \times\left(\mathbb{C} P^{1}\right)^{n-3}\right) /\left(\left(\partial \Delta_{n, 2} \times\left(\mathbb{C} P^{1}\right)^{n-3}\right) \cup\left(\Delta_{5,2} \times G_{n}\right)\right) .
$$

Using this we prove:

Theorem 12.1. The top degree homology group $H_{3 n-7}\left(G_{n, 2} / T^{n}\right)$ is isomorphic to $\mathbb{Z}$.

Proof. The dimension of the space of parameters $F_{\sigma}$ for any $P_{\sigma} \neq \Delta_{5,2}$ is by Proposition 2.22 less then or equal $2 n-8$. It implies that the dimension of $V_{2}$ is $n-1+2 n-8=3 n-9$, which further gives that $H_{3 n-7}\left(V_{2}\right)=H_{3 n-8}\left(V_{2}\right)=0$. Then the exact homology sequence of the pair $\left(G_{n, 2} / T^{n}, V_{2}\right)$ implies that $H_{3 n-7}\left(G_{n, 2} / T^{n}\right)=H_{3 n-7}\left(\left(G_{n, 2} / T^{n}\right) / V_{2}\right) \cong H_{3 n-7}\left((\stackrel{\circ}{n, 2} \times F)^{\star}\right)$. Let us consider a homology sequence of the pair $(X, Y)$ where $X=\Delta_{5,2} \times\left(\mathbb{C} P^{1}\right)^{n-3}$ and $Y=$ $\left(\partial \Delta_{n, 2} \times\left(\mathbb{C} P^{1}\right)^{n-3}\right) \cup\left(\Delta_{5,2} \times G_{n}\right)$. It holds that $H_{i}(X)=0$ for $i>2 n-6$, which implies that $H_{3 n-7}(X)=H_{3 n-8}(X)=0$. It follows from Proposition 2.19 that the dimension of $Y$ is equal to $3 n-8$ and $H_{3 n-8}(Y)=\mathbb{Z}$. Then a homology sequence gives that $H_{3 n-7}(X / Y) \cong \mathbb{Z}$, which proves the statement.

We proceed with the computation of the other homology groups for the case $n=5$, that is for the space $G_{5,2} / T^{5}$. For that purpose we will consider a filtration $V_{1} \subset V_{2} \subset V_{3}=G_{5,2} / T^{5}$ and compute the corresponding relative and absolute homology group. In the course of doing this we describe the cell decomposition of $G_{5,2} / T^{5}$.

12.1. $V_{1}$ and its homology groups. Let $V_{1}=\hat{\mu}^{-1}\left(\partial_{5,2}\right)=\cup \tilde{\mu}^{-1}\left(P_{\sigma}\right)$, where the union goes over all $P_{\sigma} \in \mathcal{P}$ such that $P_{\sigma} \subset \partial \Delta_{5,2}$. In other words, $V_{1}$ is the union of the orbit spaces of the strata whose admissible polytopes belong to $\partial \Delta_{5,2}$. The boundary $\partial \Delta_{5,2} \cong S^{3}=\cup_{i=1}^{5}\left(O_{i} \cup T_{i}\right)$, so $T_{i}$ and $O_{i}, 1 \leq i \leq 5$ provide the combinatorial decomposition of $S^{3}$. Thus, $V_{1} \cong \cup_{i=1}^{5} \hat{\mu}^{-1}\left(T_{i}\right) \cup$ $\cup_{i=1}^{5} \hat{\mu}^{-1}\left(O_{i}\right)$ and we recall that $\hat{\mu}^{-1}\left(T_{i}\right) \cong T_{i}$ and $\hat{\mu}^{-1}\left(O_{i}\right) \cong S^{5} \cong\left(O_{i} \times \mathbb{C} P^{1}\right) / \cong$, where $\partial O_{i} \times \mathbb{C} P^{1} \simeq \partial O_{i}$. Therefore, there is the continuous map $\hat{\mu}: V_{1} \rightarrow S^{3}$, but also the section $S^{3} \rightarrow V_{1}$. On $T_{i}$ this section is given by $\hat{\mu}^{-1}$, while on $O_{i}$ it is given by the composition of $\hat{\mu}^{-1}$ with the projection on a fixed parameter $* \in \mathbb{C} P^{1}$. In this way we obtain

$$
V_{1}=S^{3} \cup \cup_{i=1}^{5}\left(O_{i} \times\left(\mathbb{C} P^{1} \backslash\{*\}\right)\right) / \bumpeq, \partial O_{i} \times\left(\mathbb{C} P^{1} \backslash\{*\}\right) \simeq \partial O_{i} .
$$

Note that $\left(O_{i} \times\left(\mathbb{C} P^{1} \backslash\{*\}\right)\right) / \simeq$ is homeomorphic to $S^{5} \backslash D^{3}$, where $\simeq$ is a relation defined as in (40) and $\partial \stackrel{\circ}{D^{3}}=\partial O_{i} \subset S^{3}$. It implies that

$$
V_{1} / S^{3} \cong \vee_{5} S^{5}
$$


Lemma 12.2. The nontrivial homology groups for the space $V_{1}$ are $H_{5}\left(V_{1}\right)=\mathbb{Z}^{5}$ and $H_{3}\left(V_{1}\right)=$ $H_{0}\left(V_{1}\right)=\mathbb{Z}$.

The universal coefficient theorem implies:

Corollary 12.3. The nontrivial homology groups for $V_{1}$ with $\mathbb{Z}_{2}$-coefficients are $H_{5}\left(V_{1} ; \mathbb{Z}_{2}\right)=\mathbb{Z}_{2}^{5}$ and $H_{3}\left(V_{1} ; \mathbb{Z}_{2}\right)=H_{0}\left(V_{1} ; \mathbb{Z}_{2}\right)=\mathbb{Z}_{2}$.

Lemma 12.4. There is an induced action of the symmetric group $S_{5}$ on the homology groups for $V_{1}$ with one orbit in each homology group.

Proof. It follows from Lemma 4.7 that the symmetric group $S_{5}$ permutes the tetrahedra and permutes the octahedra which means that the sphere $S^{3}$ is invariant for this action. Therefore, there is an induced action of $S_{5}$ on the homology groups for $V_{1}$ given as follows: it acts trivially on $H_{3}\left(V_{1}\right)$ and $H_{0}\left(V_{1}\right)$, while its action on $H_{5}\left(V_{1}\right)$ is induced by the permutation of the octahedra.

12.2. $V_{2}$ and its homology groups. Let $V_{2}=\cup \tilde{\mu}^{-1}\left(P_{\sigma}\right)$, where the union goes over all admissible polytopes $P_{\sigma} \in \mathcal{P}$, which are different from $\Delta_{5,2}$. Then $V_{1} \subset V_{2}$ and set $V_{21}=V_{2} / V_{1}$. In order to compute the homology groups for $V_{21}$ we consider its filtration $L_{1} \subset L_{2} \subset V_{21}$, where subspaces $L_{1}$ and $L_{2}$ are given as follows. The space $L_{2}$ is a projection on $V_{21}$ of the union of the orbit spaces of the strata whose admissible polytopes are different from $K_{i j}(9)$ and $\Delta_{5,2}$, that is $L_{2}=\cup_{\sigma} \tilde{\mu}^{-1}\left(P_{\sigma}\right) /\left(\cup_{\sigma} \tilde{\mu}^{-1}\left(P_{\sigma}\right) \cap V_{1}\right)$, where $P_{\sigma}$ goes through admissible polytopes such that $P_{\sigma} \neq K_{i j}(9), \Delta_{5,2}$. The space $L_{1}$ is a projection on $V_{21}$ of the union of the orbit spaces of the strata over the admissible prisms and their faces, that is $L_{1}==\cup_{i=1}^{10} \tilde{\mu}^{-1}\left(P_{i}\right) /\left(\cup_{i=1}^{10} \tilde{\mu}^{-1}\left(P_{i}\right) \cap V_{1}\right)$ . Recall that there are 10 prisms, the boundary of any prism belongs to $\partial \Delta_{5,2}$ and the space of parameters for the stratum over any prism is a point. It implies that

$$
L_{1}=\vee_{10} S^{3} .
$$

It follows that the nontrivial homology groups for $L_{1}$ are $H_{3}\left(L_{1}\right)=\mathbb{Z}^{10}$ and $H_{0}\left(L_{1}\right)=\mathbb{Z}$. The universal coefficient theorem implies that the nontrivial homology groups with $\mathbb{Z}_{2}$-coefficients are $H_{3}\left(L_{1} ; \mathbb{Z}_{2}\right)=\mathbb{Z}_{2}^{10}$ and $H_{0}\left(L_{1} ; \mathbb{Z}_{2}\right)=\mathbb{Z}_{2}$.

Lemma 12.5. There is an action of $S_{5}$ on the homology groups for $L_{1}$ induced by the action of $S_{5}$ on the prisms which is given by the composition of the representation of $S_{5}$ in $S_{10}$ and the action of $S_{10}$ which permutes the prisms.

Proof. The symmetric group $S_{5}$ permutes the prisms and the corresponding strata, which implies that $S_{5}$ acts by permutations on $H_{3}\left(L_{1}\right)$ and it acts trivially on $H_{0}\left(L_{1}\right)$.

It holds $L_{1} \subset L_{2}$. Moreover, admissible polytopes for the strata from $L_{2}$ are the prisms $P_{l}$, the pyramids $K_{p q}(7)$ and the polytopes with eight vertices $K_{i j, k l}$. By Proposition 4.24 and Proposition 4.26, a facet of any of polytopes $K_{p q}(7)$ or $K_{i j, k l}$ is either $P_{l}$ either it belongs to $\partial \Delta_{5,2}$. There are altogether 25 polytopes of the types $K_{p q}(7)$ or $K_{i j, k l}$ and the space of parameters for any of these polytopes is a point. It implies that

$$
L_{2} / L_{1}=\vee_{25} S^{4} .
$$

It follows that the nontrivial homology groups for $L_{2} / L_{1}$ are $H_{4}\left(L_{2} / L_{1}\right)=\mathbb{Z}^{25}$ and $H_{0}\left(L_{2} / L_{1}\right)=$ $\mathbb{Z}$. Again by the universal coefficient theorem the nontrivial homology groups for $L_{2} / L_{1}$ with $\mathbb{Z}_{2}$-coefficients are $H_{4}\left(L_{2} / L_{1} ; \mathbb{Z}_{2}\right)=\mathbb{Z}_{2}^{25}$ and $H_{0}\left(L_{2} / L_{1} ; \mathbb{Z}_{2}\right)=\mathbb{Z}_{2}$. 
Lemma 12.6. There is an induced action of the symmetric group $S_{5}$ on the homology groups for $L_{2} / L_{1}$ which has two orbits .

Proof. The symmetric groups $S_{5}$ permutes $K_{p q}(7)$, permutes $K_{i j, k l}$ and the corresponding strata. Therefore, there is an induced action of $S_{5}$ on $H_{4}\left(L_{2} / L_{1}\right)$ which is given by the permutation of elements which are determined by $K_{p q}(7)$ and by the permutation of elements which are determined by $K_{i j, k l}$. Thus, there are two orbits for $S_{5}$-action, one consisting of 10 elements and the other one consisting of 15 elements.

Lemma 12.7. The nontrivial homology groups for $L_{2}$ are $H_{4}\left(L_{2}\right)=\mathbb{Z}^{15}$ and $H_{0}\left(L_{2}\right)=\mathbb{Z}$.

Proof. The exact homology sequence of the pair $\left(L_{2}, L_{1}\right)$ gives directly that $H_{1}\left(L_{2}\right)=H_{2}\left(L_{2}\right)=$ $H_{i}\left(L_{2}\right)=0$ for $i \geq 5$. In order to determine $H_{3}\left(L_{2}\right)$ and $H_{4}\left(L_{2}\right)$ we consider a cell decomposition for $L_{2}$. The three dimensional cells in this cell decomposition are given by the admissible prisms $P_{l}$ over six vertices, while the four-dimensional cells are given by the admissible polytopes $K_{i j, k l}$ and $K_{p q}(7)$. A facet of $K_{p q}(7)$ which belongs to $\stackrel{\circ}{\Delta}, 2_{5}$ is some prism $P_{l}$. It implies that the boundary in $L_{2}$ of the cell $K_{p q}(7)$ consists of the three-dimensional cell $P_{l}$ and a point. The number of the pyramids $K_{p q}(7)$ is 10 and it is equal to the number of the prisms $P_{l}$ and, obviously, different pyramids $K_{p q}(7)$ have different prisms in their boundaries. In this way we deduce that $H_{3}\left(L_{2}\right)=0$. Then the exact homology sequence implies that $H_{4}\left(L_{2}\right)=\mathbb{Z}^{15}$.

By the universal coefficient theorem it follows:

Corollary 12.8. The nontrivial homology groups for $L_{2}$ with $\mathbb{Z}_{2}$-coefficients are $H_{4}\left(L_{2} ; \mathbb{Z}_{2}\right)=\mathbb{Z}_{2}^{15}$ and $H_{0}\left(L_{2} ; \mathbb{Z}_{2}\right)=\mathbb{Z}_{2}$.

Lemma 12.9. The symmetric group $S_{5}$ acts on $H_{4}\left(L_{2}\right)$ by permutations and this action is induced by the $S_{5}$-action on the set $\left\{K_{i j, k l}\right\}$.

The union $K$ of the orbit spaces of the 10 strata over $\stackrel{\circ}{K}_{i j}(9), 1 \leq i<j \leq 5$ is a dense set in $V_{21}$ and by Proposition 4.23, $K=V_{21} \backslash L_{2}$. It implies that $K^{\star}=V_{21} / L_{2}$ is an one-point compactification of $K$.

Recall that the orbit space of each of these strata is homeomorphic to $K_{i j}^{\circ}(9) \times \mathbb{C} P_{A}^{1}$, where $A=\{(1: 0),(0: 1),(1: 1)\}$.

Lemma 12.10. The one-point compactification $K_{i j}^{\star}(9)$ of the orbit space $K_{i j}(9) \times \mathbb{C} P_{A}^{1}$ in $V_{21} / L_{2}$ is given by

$$
\left(K_{i j}(9) \times \mathbb{C} P^{1}\right) / \simeq \text {, where } \partial K_{i j}(9) \times \mathbb{C} P^{1} \simeq K_{i j}(9) \times A \backsim * .
$$

Moreover,

$$
K_{i j}^{\star}(9) \cong S^{6} \vee S^{5} \vee S^{5} \text {. }
$$

Proof. The statement follows from the observation that $K_{i j}(9) \times \mathbb{C} P^{1}$ is a compactification of $K_{i j}^{\circ}(9) \times \mathbb{C} P_{A}^{1}$ and $\left(K_{i j}(9) \times \mathbb{C} P^{1}\right) \backslash\left(K_{i j}^{\circ}(9) \times \mathbb{C} P_{A}^{1}\right)=\left(\partial K_{i j}(9) \times \mathbb{C} P^{1}\right) \cup\left(K_{i j}(9) \times A\right)$. We want to emphasize that the one-point compactification $K_{i j}^{\star}(9)$ of $\stackrel{\circ}{K}_{i j}(9) \times \mathbb{C} P_{A}^{1}$ in $V_{21} / L_{2}$ has an explicit geometric realization. We demonstrate it for the admissible polytope $K_{12}(9)$ and due to the action of the symmetric group $S_{5}$, it will then hold for any other polytope $K_{i j}(9)$. It 
follows from Proposition 4.23 that the boundary of the stratum over $K_{12}(9)$ contains the fourdimensional strata over the polytopes $K_{12,34}, K_{12,35}, K_{12,45}, K_{34}(7), K_{35}(7)$ and $K_{45}(7)$. It holds $K_{12,34} \cup K_{34}(7)=K_{12,35} \cup K_{35}(7)=K_{12,45} \cup K_{45}(7)=K_{12}$. Moreover, since the stratum over $K_{12}(9)$ is parametrized by $\mathbb{C} P_{A}^{1}$, it directly follows that the pairs $\left(K_{12,34}, K_{34}(7)\right),\left(K_{12,35}, K_{35}(7)\right)$ and $\left(K_{12}, K_{45}(7)\right)$ are parametrized by the points $(1: 0),(0: 1)$ and $(1: 1)$, respectively. In addition any stratum over a face of $K_{12}(9)$ is in the boundary of the stratum over $K_{12}(9)$ which completes the proof. The second statement (42) follows from the observation that $K_{i j}^{\star}(9) \cong S^{3} *$ $\left(K_{i j}(9) \times \mathbb{C} P^{1}\right) /\left(K_{i j}(9) * A\right) \cong S^{3} *\left(S^{2} \vee S^{1} \vee S^{1}\right) \cong S^{6} \vee S^{5} \vee S^{5}$.

Remark 12.11. We will further use a cell decomposition for $K_{i j}^{\star}(9)$ which is for our purposes more geometrical, that is $K_{i j}^{\star}(9) \cong \stackrel{\circ}{K}_{i j}(9) \times\left(\left(S^{2} \vee S^{1} \vee S^{1}\right) \backslash\{*\}\right) \cup\{*\}$, which implies that the six-cell is $e_{i j}=\stackrel{\circ}{K}_{i j}(9) \times\left(S^{2} \backslash\{*\}\right)$, while the five-cells are $f_{i j}^{l}=\stackrel{\circ}{K}_{i j}(9) \times p_{l}$, where $p_{1}$ and $p_{2}$ are non-intersecting paths in $\mathbb{C} P^{1}$ such that $p_{1}$ connects the points $(0: 1)$ and $(1: 1)$ and $p_{2}$ connects the points $(1: 1)$ and $(1: 0)$. Note that the cells $e_{i j}$ and $f_{i j}^{1}, f_{i j}^{2}$ represent six-dimensional and five-dimensional cycles in $K_{i j}^{\star}$.

It follows from (41) that

$$
V_{21} / L_{2}=\cup_{1 \leq i<j \leq 5}\left(K_{i j}(9) \times \mathbb{C} P^{1}\right) / \simeq, \quad \text { where } \partial K_{i j}(9) \times \mathbb{C} P^{1} \simeq K_{i j}(9) \times A \backsim * .
$$

Then (42) implies that

$$
V_{21} / L_{2} \cong\left(\vee_{10} S^{6}\right) \vee\left(\vee_{20} S^{5}\right)
$$

The description of the nontrivial homology groups for $V_{21} / L_{2}$ directly follows from (43):

$$
H_{6}\left(V_{21} / L_{2}\right)=\mathbb{Z}^{10}, H_{5}\left(V_{21} / L_{2}\right)=\mathbb{Z}^{20} \text { and } H_{0}\left(V_{21} / L_{2}\right)=\mathbb{Z} \text {. }
$$

Remark 12.12. The symmetric group $S_{5}$ acts on the set of the orbit spaces $\stackrel{\circ}{K}_{i j}(9) \times \mathbb{C} P_{A}^{1}$ by permutations. We assume the expression (43) to be invariant under this action. Then there is an induced action of $S_{5}$ on the homology groups for $V_{21} / L_{2}$. Note that this action on $H_{6}\left(V_{21} / L_{2}\right)$ has one orbit, while on $H_{5}\left(V_{21} / L_{2}\right)$ it has two orbits each of them containing 10 elements.

In order to avoid an orientation issue we compute further the homology groups with coefficients in $\mathbb{Z}_{2}$.

Proposition 12.13. The nontrivial homology groups for $V_{21}$ with $\mathbb{Z}_{2}$-coefficients are:

$$
H_{6}\left(V_{21} ; \mathbb{Z}_{2}\right)=\mathbb{Z}_{2}^{10}, \quad H_{5}\left(V_{21} ; \mathbb{Z}_{2}\right)=\mathbb{Z}_{2}^{5}, \quad H_{4}\left(V_{21} ; \mathbb{Z}_{2}\right)=\mathbb{Z}_{2} \text { and } H_{0}\left(V_{21} ; \mathbb{Z}_{2}\right)=\mathbb{Z}_{2} \text {. }
$$

Proof. Since $H_{i}\left(L_{2}\right)=0$ for $i \geq 5$, the exact homology sequence of the pair $\left(V_{21}, L_{2}\right)$ directly implies that $H_{i}\left(V_{21}\right)=0$ for $i \geq 7$ and $H_{6}\left(V_{21}\right)=\mathbb{Z}^{10}$. In order to determine $H_{5}\left(V_{21}\right)$ we note that all five-dimensional cycles in $V_{21}$ comes from $V_{21} / L_{2}$, since $L_{2}$ has no five-dimensional cells. It follows from Remark 12.11 that the five dimensional cycles in $V_{21} / L_{2}$ are of the form $\sum_{1 \leq i<j \leq 5}\left(\alpha_{i j}^{1} f_{i j}^{1}+\alpha_{i j}^{2} f_{i j}^{2}\right)$. The boundaries $\partial_{4}^{V_{21}} f_{i j}^{l}, l=1,2$, in $V_{21}$ are four-dimensional cycles consisting of the orbit spaces of four-dimensional strata in the boundary of the strata over $\stackrel{\circ}{K}_{i j}(9)$. Note that we do not need to take care about an orientation issue since we work with $\mathbb{Z}_{2}$-coefficients. It follows from Proposition 6.1, Proposition 6.2 and Proposition 6.3 and Theorem 9.14 that these boundaries are as follows:

$$
f_{12}^{1} \rightarrow \stackrel{\circ}{K}_{12,34}+\stackrel{\circ}{K}_{12,45}+\stackrel{\circ}{K}_{34}+\stackrel{\circ}{K}_{45}, f_{12}^{2} \rightarrow \stackrel{\circ}{K}_{12,45}+\stackrel{\circ}{K}_{12,35}+\stackrel{\circ}{K}_{45}+\stackrel{\circ}{K}_{35},
$$




$$
\begin{aligned}
& f_{13}^{1} \rightarrow \stackrel{\circ}{K}_{13,24}+\stackrel{\circ}{K}_{13,45}+\stackrel{\circ}{K}_{24}+\stackrel{\circ}{K}_{45}, f_{13}^{2} \rightarrow \stackrel{\circ}{K}_{13,45}+\stackrel{\circ}{K}_{13,25}+\stackrel{\circ}{K}_{45}+\stackrel{\circ}{K}_{25}, \\
& f_{14}^{1} \rightarrow \stackrel{\circ}{K}_{14,23}+\stackrel{\circ}{K}_{14,35}+\stackrel{\circ}{K}_{23}+\stackrel{\circ}{K}_{35}, f_{14}^{2} \rightarrow \stackrel{\circ}{K}_{14,35}+\stackrel{\circ}{K}_{14,25}+\stackrel{\circ}{K}_{35}+\stackrel{\circ}{K}_{25}, \\
& f_{15}^{1} \rightarrow \stackrel{\circ}{K}_{15,23}+\stackrel{\circ}{K}_{15,34}+\stackrel{\circ}{K}_{23}+\stackrel{\circ}{K}_{34}, f_{15}^{2} \rightarrow \stackrel{\circ}{K}_{15,34}+\stackrel{\circ}{K}_{15,24}+\stackrel{\circ}{K}_{34}+\stackrel{\circ}{K}_{24}, \\
& f_{23}^{1} \rightarrow \stackrel{\circ}{K}_{15,23}+\stackrel{\circ}{K}_{23,45}+\stackrel{\circ}{K}_{15}+\stackrel{\circ}{K}_{45}, f_{23}^{2} \rightarrow \stackrel{\circ}{K}_{23,45}+\stackrel{\circ}{K}_{14,23}+\stackrel{\circ}{K}_{45}+\stackrel{\circ}{K}_{14}, \\
& f_{24}^{1} \rightarrow \stackrel{\circ}{K}_{15,24}+\stackrel{\circ}{K}_{24,35}+\stackrel{\circ}{K}_{15}+\stackrel{\circ}{K}_{35}, f_{24}^{2} \rightarrow \stackrel{\circ}{K}_{24,35}+\stackrel{\circ}{K}_{13,24}+\stackrel{\circ}{K}_{35}+\stackrel{\circ}{K}_{13}, \\
& f_{25}^{1} \rightarrow \stackrel{\circ}{K}_{14,25}+\stackrel{\circ}{K}_{25,34}+\stackrel{\circ}{K}_{14}+\stackrel{\circ}{K}_{34}, f_{25}^{2} \rightarrow \stackrel{\circ}{K}_{25,34}+\stackrel{\circ}{K}_{25,13}+\stackrel{\circ}{K}_{34}+\stackrel{\circ}{K}_{13}, \\
& f_{34}^{1} \rightarrow \stackrel{\circ}{K}_{15,34}+\stackrel{\circ}{K}_{12,34}+\stackrel{\circ}{K}_{15}+\stackrel{\circ}{K}_{12}, f_{34}^{2} \rightarrow \stackrel{\circ}{K}_{12,34}+\stackrel{\circ}{K}_{25,34}+\stackrel{\circ}{K}_{12}+\stackrel{\circ}{K}_{25}, \\
& f_{35}^{1} \rightarrow \stackrel{\circ}{K}_{14,35}+\stackrel{\circ}{K}_{12,35}+\stackrel{\circ}{K}_{14}+\stackrel{\circ}{K}_{12}, f_{35}^{2} \rightarrow \stackrel{\circ}{K}_{12,35}+\stackrel{\circ}{K}_{24,35}+\stackrel{\circ}{K}_{12}+\stackrel{\circ}{K}_{24}, \\
& f_{45}^{1} \rightarrow \stackrel{\circ}{K}_{23,45}+\stackrel{\circ}{K}_{12,45}+\stackrel{\circ}{K}_{23}+\stackrel{\circ}{K}_{12}, f_{45}^{2} \rightarrow \stackrel{\circ}{K}_{12,45}+\stackrel{\circ}{K}_{13,45}+\stackrel{\circ}{K}_{12}+\stackrel{\circ}{K}_{13} .
\end{aligned}
$$

The generators for $H_{4}\left(L_{2}\right)$ are by the proof of Lemma12.7 given by $g_{i j, k l}=\stackrel{\circ}{K}_{i j, k l}+\stackrel{\circ}{K}_{i j}(7)+\stackrel{\circ}{K}_{k l}$ (7). In terms of these generators the above boundaries can be written as follows:

$$
\begin{aligned}
& f_{12}^{1} \rightarrow g_{12,34}+g_{12,45}, f_{12}^{2} \rightarrow g_{12,45}+g_{12,35}, f_{13}^{1} \rightarrow g_{13,24}+g_{13,45}, f_{13}^{2} \rightarrow g_{13,45}+g_{13,25}, \\
& f_{14}^{1} \rightarrow g_{14,23}+g_{14,35}, f_{14}^{2} \rightarrow g_{14,35}+g_{14,25}, f_{15}^{1} \rightarrow g_{15,23}+g_{15,34}, f_{15}^{2} \rightarrow g_{15,34}+g_{15,24}, \\
& f_{23}^{1} \rightarrow g_{15,23}+g_{23,45}, f_{23}^{2} \rightarrow g_{23,45}+g_{23,14}, f_{24}^{1} \rightarrow g_{15,24}+g_{24,35}, f_{24}^{2} \rightarrow g_{24,35}+g_{13,24}, \\
& f_{25}^{1} \rightarrow g_{14,25}+g_{25,34}, f_{25}^{2} \rightarrow g_{25,34}+g_{25,13}, f_{34}^{1} \rightarrow g_{15,34}+g_{12,34}, f_{34}^{2} \rightarrow g_{12,34}+g_{25,34}, \\
& f_{35}^{1} \rightarrow g_{14,35}+g_{12,35}, f_{35}^{2} \rightarrow g_{12,35}+g_{24,35}, f_{45}^{1} \rightarrow g_{23,45}+g_{12,45}, f_{45}^{2} \rightarrow g_{12,45}+g_{13,45} .
\end{aligned}
$$

Now, searching for five-dimensional cycles in $V_{21}$ we consider the system:

$$
\partial_{4}^{V_{21}}\left(\sum_{1 \leq i<j \leq 5}\left(\alpha_{i j}^{1} f_{i j}^{1}+\alpha_{i j}^{2} f_{i j}^{2}\right)\right)=\sum_{1 \leq i<\leq 5} \alpha_{i j}^{1} \partial_{4}^{V_{21}} f_{i j}^{1}+\sum_{1 \leq i<j \leq 5} \alpha_{i j}^{2} \partial_{4}^{V_{21}} f_{i j}^{2}=0 .
$$

Substituting the above expressions for the boundaries in the system (44) we obtain:

$$
\begin{gathered}
\alpha_{12}^{1}+\alpha_{34}^{1}+\alpha_{34}^{2}=0, \alpha_{12}^{1}+\alpha_{12}^{2}+\alpha_{45}^{1}+\alpha_{45}^{2}=0, \alpha_{12}^{2}+\alpha_{35}^{1}+\alpha_{35}^{2}=0, \alpha_{13}^{1}+\alpha_{24}^{2}=0, \\
\alpha_{13}^{1}+\alpha_{13}^{2}+\alpha_{45}^{2}=0, \alpha_{13}^{2}+\alpha_{25}^{2}=0, \alpha_{14}^{1}+\alpha_{23}^{2}=0, \alpha_{14}^{1}+\alpha_{14}^{2}+\alpha_{35}^{1}=0, \\
\alpha_{14}^{2}+\alpha_{25}^{1}=0, \alpha_{15}^{1}+\alpha_{23}^{1}=0, \alpha_{15}^{1}+\alpha_{15}^{2}+\alpha_{34}^{1}=0, \alpha_{15}^{2}+\alpha_{24}^{1}=0, \\
\alpha_{23}^{1}+\alpha_{23}^{2}+\alpha_{45}^{1}=0, \alpha_{24}^{1}+\alpha_{24}^{2}+\alpha_{35}^{2}=0, \alpha_{25}^{1}+\alpha_{25}^{2}+\alpha_{34}^{2}=0 .
\end{gathered}
$$

The solution of this system is given by

$$
\begin{gathered}
\alpha_{45}^{1}=\alpha_{13}^{1}+\alpha_{13}^{2}, \alpha_{45}^{2}=\alpha_{12}^{1}+\alpha_{12}^{2}+\alpha_{13}^{1}+\alpha_{13}^{2}, \quad \alpha_{35}^{2}=\alpha_{12}^{2}+\alpha_{14}^{1}+\alpha_{14}^{2}, \alpha_{35}^{1}=\alpha_{14}^{1}+\alpha_{14}^{2}, \\
\alpha_{34}^{2}=\alpha_{13}^{2}+\alpha_{14}^{2}, \alpha_{34}^{1}=\alpha_{12}^{1}+\alpha_{13}^{2}+\alpha_{14}^{2}, \alpha_{25}^{2}=\alpha_{13}^{2}, \alpha_{25}^{1}=\alpha_{14}^{2}, \alpha_{24}^{2}=\alpha_{13}^{1}, \\
\alpha_{24}^{1}=\alpha_{12}^{2}+\alpha_{13}^{1}+\alpha_{14}^{1}+\alpha_{14}^{2}, \alpha_{23}^{2}=\alpha_{14}^{1}, \alpha_{23}^{1}=\alpha_{12}^{1}+\alpha_{12}^{2}+\alpha_{13}^{1}+\alpha_{13}^{2}+\alpha_{14}^{1}, \\
\alpha_{15}^{2}=\alpha_{12}^{2}+\alpha_{13}^{1}+\alpha_{14}^{1}+\alpha_{14}^{2}, \alpha_{15}^{1}=\alpha_{12}^{1}+\alpha_{12}^{2}+\alpha_{13}^{1}+\alpha_{13}^{2}+\alpha_{14}^{1},
\end{gathered}
$$

where $\alpha_{12}^{1}, \alpha_{12}^{2}, \alpha_{13}^{1}, \alpha_{13}^{2}, \alpha_{14}^{1}, \alpha_{14}^{2}$ are free variables. Thus, the dimension of the solution space of this system is 6 , which implies that $H_{5}\left(V_{21} ; \mathbb{Z}_{2}\right)=\mathbb{Z}_{2}^{6}$. It further implies that the image of the map $H_{5}\left(V_{21} / L_{2} ; \mathbb{Z}_{2}\right) \rightarrow H_{4}\left(L_{2}, Z_{2}\right)$ is $\mathbb{Z}_{2}^{14}$. Thus, if we now consider the exact homology sequence $H_{5}\left(V_{21} / L_{2} ; \mathbb{Z}_{2}\right) \rightarrow H_{4}\left(L_{2} ; \mathbb{Z}_{2}\right)=\mathbb{Z}_{2}^{15} \rightarrow H_{4}\left(V_{21}, \mathbb{Z}_{2}\right) \rightarrow 0=H_{4}\left(V_{21} / L_{2}, \mathbb{Z}_{2}\right)$, we deduce that $H_{4}\left(V_{21}, \mathbb{Z}_{2}\right)=\mathbb{Z}_{2}$. Moreover, as a generator for $H_{4}\left(V_{21}, \mathbb{Z}_{2}\right)$ can be taken any of generators $g_{i j, k l}$ for $H_{4}\left(L_{2}\right)$. 
Proposition 12.14. The non-trivial homology groups for $V_{2}$ with coefficients in $\mathbb{Z}_{2}$ are $H_{6}\left(V_{2} ; \mathbb{Z}_{2}\right)=$ $\mathbb{Z}_{2}^{6}, H_{5}\left(V_{2} ; \mathbb{Z}_{2}\right)=\mathbb{Z}_{2}^{7}$ and $H_{0}\left(V_{2} ; \mathbb{Z}_{2}\right)=\mathbb{Z}_{2}$.

Proof. Combining the exact homology sequence of the pair $\left(V_{2}, V_{1}\right)$ and Lemma 12.13 we obtain that $H_{i}\left(V_{2} ; \mathbb{Z}_{2}\right)=0$ for $i \geq 7$. Moreover, $V_{2}$ has no cells of the dimension greater or equal then 7. The six-dimensional cycles in $V_{2}$ comes from $V_{21}$ since $V_{1}$ has no six-dimensional cells. These cycles are, by Remark 12.11, given by $e_{i j}=\stackrel{\circ}{K}_{i j}(9) \times\left(S^{2} \backslash\{*\}\right)$, where $1 \leq i<j \leq 5$. Then $\partial_{5}^{V_{2}}\left(e_{i j}\right)=\partial_{5}^{V_{21}}\left(e_{i j}\right)+\partial_{5}^{V_{1}}\left(e_{i j}\right)=0+\partial_{5}^{V_{1}}\left(e_{i j}\right)$. The five-dimensional cycles in $V_{1}$ are cycles which correspond to the five-spheres over the octahedra on $\partial \Delta_{5,2}$. They represent a basis for $H_{5}\left(V_{1} ; \mathbb{Z}_{2}\right)$ and we denote them by $S_{i}, 1 \leq i \leq 5$. It follows from Proposition 4.23 that the boundary of $K_{i j}(9)$ contains exactly the octahedra $O_{i}$ and $O_{j}$. Note that, since we work with $\mathbb{Z}_{2}$-coefficients, we do not need to take care about an orientation. We obtain that $\partial_{5}^{V_{1}}\left(e_{i j}\right)$ are as follows:

$$
\begin{gathered}
e_{12} \rightarrow S_{1}+S_{2}, e_{13} \rightarrow S_{1}+S_{3}, e_{14} \rightarrow S_{1}+S_{4}, e_{15} \rightarrow S_{1}+S_{5}, \\
e_{23} \rightarrow S_{2}+S_{3}, e_{24} \rightarrow S_{2}+S_{4}, e_{25} \rightarrow S_{2}+S_{5}, \\
e_{34} \rightarrow S_{3}+S_{4}, e_{35} \rightarrow S_{3}+S_{5}, e_{45} \rightarrow S_{4}+S_{5} .
\end{gathered}
$$

We consider homogeneous system of five linear equations given by

$$
\sum_{1 \leq i<j \leq 5} \alpha_{i j} \partial_{6} e_{i j}=\sum_{1 \leq i<j \leq 5} \alpha_{i j}\left(S_{i}+S_{j}\right)=0,
$$

and it can be directly checked that its solution space has the dimension 4. More precisely, the space of solutions of this system is given by

$$
\begin{array}{ll}
\alpha_{15}=\alpha_{12}+\alpha_{13}+\alpha_{14}, & \alpha_{25}=\alpha_{12}+\alpha_{23}+\alpha_{24}, \\
\alpha_{35}=\alpha_{13}+\alpha_{23}+\alpha_{34}, & \alpha_{45}=\alpha_{14}+\alpha_{24}+\alpha_{34} .
\end{array}
$$

where $\alpha_{12}, \alpha_{13}, \alpha_{14}, \alpha_{23}, \alpha_{24}$ and $\alpha_{34}$ are free $\mathbb{Z}_{2}$-variables.

It implies that $H_{6}\left(V_{2} ; \mathbb{Z}_{2}\right) \cong \mathbb{Z}_{2}^{6}$ and that the generators for $H_{6}\left(V_{2} ; \mathbb{Z}_{2}\right)$ are given by the cycles

$$
\begin{array}{lll}
c_{12}=e_{12}+e_{15}+e_{25}, & c_{13}=e_{13}+e_{15}+e_{35}, & c_{14}=e_{14}+e_{15}+e_{45}, \\
c_{23}=e_{23}+e_{25}+e_{35}, & c_{24}=e_{24}+e_{25}+e_{45}, & c_{34}=e_{34}+e_{35}+e_{45} .
\end{array}
$$

Therefore, the quotient $H_{5}\left(V_{1} ; \mathbb{Z}_{2}\right) / \operatorname{Im} \partial_{5}\left(H_{6}\left(V_{21} ; \mathbb{Z}_{2}\right)\right.$ is isomorphic to $\mathbb{Z}_{2}$.

Now the exact homology sequence $H_{6}\left(V_{21} ; \mathbb{Z}_{2}\right) \rightarrow H_{5}\left(V_{1} ; \mathbb{Z}_{2}\right) \rightarrow H_{5}\left(V_{2} ; \mathbb{Z}_{2}\right) \rightarrow H_{5}\left(V_{21} ; \mathbb{Z}_{2}\right) \cong$ $\mathbb{Z}_{2}^{6} \rightarrow 0$ implies that $H_{5}\left(V_{2} ; \mathbb{Z}_{2}\right)=\mathbb{Z}_{2}^{7}$. Further, the map $\mathbb{Z}_{2} \cong H_{4}\left(V_{21} ; \mathbb{Z}_{2}\right) \rightarrow H_{3}\left(V_{1} ; \mathbb{Z}_{2}\right) \cong \mathbb{Z}_{2}$ is an isomorphism, since the generator for $H_{3}\left(V_{1} ; \mathbb{Z}_{2}\right)$ is given by $\partial \Delta_{5,2}$, while the generator for $H_{4}\left(V_{21} ; \mathbb{Z}_{2}\right)$ is given by, for example, $g_{12,34}$ whose boundary is $\partial \Delta_{5,2}$. Then the exact homology sequence implies that $H_{4}\left(V_{2}, \mathbb{Z}_{2}\right)=0$. The fact that $H_{i}\left(V_{2} ; \mathbb{Z}_{2}\right)=0$ for $i \neq 0,4,5,6$ follows also directly from the exact homology sequence.

Corollary 12.15. The space $V_{21}$ has no torsion in homology.

Proof. By the universal coefficient formula we have that $H_{i}\left(V_{21}\right)=0,1 \leq i \leq 3, H_{i}\left(V_{2}\right)=0$, $1 \leq i \leq 4, H_{5}\left(V_{21}\right) \cong \mathbb{Z}^{l} \oplus \mathbb{Z}_{2}^{q}$, where $l+q=6$ and $H_{6}\left(V_{21}\right)=\mathbb{Z}^{l} \oplus \mathbb{Z}_{2}^{q}$, where $l+q=10$. Then an exact homotopy sequence of the pair $\left(V_{2}, V_{1}\right)$ implies $H_{4}\left(V_{21}\right) \cong \mathbb{Z}$, while an exact homotopy sequence of the pair $\left(V_{21}, L_{2}\right)$ implies that $H_{5}\left(V_{21}\right) \cong \mathbb{Z}^{6}$ and $H_{6}\left(V_{21}\right) \cong \mathbb{Z}^{10}$.

Theorem 12.16. The non-trivial homology groups of the space $V_{2}$ are $H_{6}\left(V_{2}\right) \cong \mathbb{Z}^{5}, H_{5}\left(V_{2}\right) \cong$ $\mathbb{Z}^{6} \oplus \mathbb{Z}_{2}$ and $H_{0}\left(V_{2}\right) \cong \mathbb{Z}$. 
Proof. We follow the proof of Proposition 12.14. Note that the boundary $\partial_{5}^{V_{1}}\left(e_{i j}\right)$ with integral coefficients is the same as with $\mathbb{Z}_{2}$-coefficients. It follows from the observation that this boundary is given by the octahedra $O_{i}$ and $O_{j}$ of $K_{i j}$, while it is identity on the parameter space $S^{2} \backslash\{*\}$. Moreover, being a facet of $\Delta_{5,2}$, an orientation of the octahedra $O_{i}$ is given by an exterior normal for $\Delta_{5,2}$, so it coincides with the induced orientation from $K_{i j}$ for any $K_{i j}, i \neq j$, since $K_{i j} \subset \Delta_{5,2}$. Therefore, the 6 -cycles in $V_{2}$ are given by the solution of (45) with integer coefficients. The solution space of this system has dimension 5 and it is given by

$$
\begin{gathered}
\alpha_{15}=-\alpha_{12}-\alpha_{13}-\alpha_{14}, \quad \alpha_{25}=-\alpha_{12}-\alpha_{23}-\alpha_{24}, \\
\alpha_{35}=\alpha_{12}+\alpha_{14}+\alpha_{24}, \quad \alpha_{45}=\alpha_{12}+\alpha_{13}+\alpha_{23} . \\
\alpha_{34}=-\alpha_{12}-\alpha_{13}-\alpha_{14}-\alpha_{23}-\alpha_{24}
\end{gathered}
$$

where $\alpha_{12}, \alpha_{13}, \alpha_{14}, \alpha_{23}, \alpha_{24}$ are free integer variables. Therefore, $H_{6}\left(V_{2}\right) \cong \mathbb{Z}^{5}$ and $\operatorname{Im} \partial_{5}\left(H_{6}\left(V_{21}\right)\right.$ is a span of $S_{1}+S_{2}, S_{1}+S_{3}, S_{1}+S_{4}, S_{1}+S_{5}, S_{2}+S_{3}$, which coincides with a span of $2 S_{1}, S_{1}+$ $S_{2}, S_{1}+S_{3}, S_{1}+S_{4}, S_{1}+S_{5}$. It implies that $H_{5}\left(V_{1}\right) / \operatorname{Im} \partial_{5}\left(H_{6}\left(V_{21}\right) \cong \mathbb{Z}_{2}\right.$. Now an exact homology sequence $H_{6}\left(V_{21}\right) \rightarrow H_{5}\left(V_{1}\right) \rightarrow H_{5}\left(V_{2}\right) \rightarrow H_{5}\left(V_{21}\right) \cong \mathbb{Z}^{6} \rightarrow 0$ implies that $H_{5}\left(V_{2}\right)=\mathbb{Z}^{6} \oplus \mathbb{Z}_{2}$, which completes the proof.

12.3. The homology groups for $V_{3}=G_{5,2} / T^{5}$. We denoted by $V_{2}$ the union of the orbit spaces of the strata which are different from the main stratum. As we already pointed, $V_{3} / V_{2}$ is an one-point compactification of $W / T^{5}$, which implies

$$
\begin{gathered}
V_{3} / V_{2} \cong\left(\stackrel{\circ}{\Delta}_{5,2} \times F\right)^{\star} \cong\left(\Delta_{5,2} \times U\right) /\left(U_{1} \cup U_{2}\right), \\
U=\mathbb{C} P^{1} \times \mathbb{C} P^{1}, \quad U_{1}=\partial \Delta_{5,2} \times \mathbb{C} P^{1} \times \mathbb{C} P^{1}, \\
U_{2}=\left(\Delta_{5,2} \times A \times \mathbb{C} P^{1}\right) \cup\left(\Delta_{5,2} \times \mathbb{C} P^{1} \times A\right) \cup\left(\Delta_{5,2} \times \Delta\left(\mathbb{C} P^{1}\right)\right) .
\end{gathered}
$$

Note that $X_{1}=\left(\Delta_{5,2} \times U\right) / U_{1} \cong S^{3} * U$. We denote by $X_{2}$ the projection of $U_{2}$ on $X_{1}$, that is $X_{2}=U_{2} /\left(U_{1} \cap U_{2}\right) \cong S^{3} * U_{2}$. We obtain that

$$
X_{1} / X_{2} \cong\left(S^{3} * U\right) /\left(S^{3} * U_{2}\right) \cong\left(S^{3} *\left(U / U_{2}\right)\right) /\left(S^{3} *\{\mathrm{pt}\}\right) .
$$

It follows that

$$
V_{3} / V_{2} \cong S^{3} *\left(U / U_{2}\right)
$$

Proposition 12.17. The non-trivial homology groups for $V_{3} / V_{2}$ are as follows:

$$
H_{8}\left(V_{3} / V_{2}\right)=H_{0}\left(V_{3} / V_{2}\right)=\mathbb{Z}, \quad H_{7}\left(V_{3} / V_{2}\right)=\mathbb{Z}^{5}, \quad H_{6}\left(V_{3} / V_{2}\right)=\mathbb{Z}^{6} .
$$

Proof. Since $\bar{H}_{i}\left(S^{3} *\left(U / U_{2}\right)\right)=H_{i-1}\left(\left(S^{3} \times\left(U / U_{2}\right)\right) /\left(S^{3} \vee\left(U / U_{2}\right)\right)\right)$, it follows that

$$
\bar{H}_{i}\left(S^{3} *\left(U / U_{2}\right)\right)=H_{i-1}\left(S^{3} \times\left(U / U_{2}\right)\right) /\left(H_{i-1}\left(S^{3}\right) \oplus H_{i-1}\left(U / U_{2}\right)\right) .
$$

In order to compute the homology groups for $U / U_{2}$, we first compute the homology groups for $U_{2}$. Let us set $U_{2}=U_{3} \cup U_{4}$ for $U_{3}=\left(\Delta_{5,2} \times A \times \mathbb{C} P^{1}\right) \cup\left(\Delta_{5,2} \times \mathbb{C} P^{1} \times A\right)$ and $U_{4}=\Delta_{5,2} \times$ $\left.\Delta\left(\mathbb{C} P^{1}\right)\right)$. The homology groups for $U_{3}$ we compute by applying the Mayer-Vietoris sequence to the pair $\left(\mathbb{C} P^{1} \times A, A \times \mathbb{C} P^{1}\right)$. Since $\left(\mathbb{C} P^{1} \times A\right) \cap\left(A \times \mathbb{C} P^{1}\right)=A \times A$, it follows that $H_{i}\left(U_{3}\right)=H_{i}\left(\mathbb{C} P^{1} \times A\right) \oplus H_{i}\left(A \times \mathbb{C} P^{1}\right)$ for $i \geq 2$. The exact sequence $0 \rightarrow H_{1}\left(U_{3}\right) \rightarrow$ $H_{0}(A \times A) \rightarrow H_{0}\left(A \times \mathbb{C} P^{1}\right) \oplus H_{0}\left(\mathbb{C} P^{1} \times A\right) \rightarrow H_{0}\left(U_{3}\right)$ gives the exact sequence $0 \rightarrow H_{1}\left(U_{3}\right) \rightarrow$ 
$\mathbb{Z}^{9} \rightarrow \mathbb{Z}^{3} \oplus \mathbb{Z}^{3} \rightarrow Z$, which implies that $H_{1}\left(U_{3}\right) \cong \mathbb{Z}^{4}$. Thus, the nontrivial homology groups for $U_{3}$ are given by

$$
H_{i}\left(U_{3}\right)=\left\{\begin{array}{cc}
\mathbb{Z}^{3} \oplus \mathbb{Z}^{3}, & i=2 \\
\mathbb{Z}^{4}, & i=1 \\
\mathbb{Z}, & i=0 .
\end{array}\right.
$$

Now we apply the Mayer-Vietoris sequence to the pair $\left(U_{3}, U_{4}\right)$. Since $U_{3} \cap U_{4}=\Delta(A)$, it follows $H_{i}\left(U_{2}\right)=H_{i}\left(U_{3}\right) \oplus H_{i}\left(U_{4}\right)$ for $i \geq 2$. We further obtain the exact sequence $0 \rightarrow H_{1}\left(U_{3}\right) \oplus$ $H_{1}\left(U_{4}\right) \rightarrow H_{1}\left(U_{2}\right) \rightarrow H_{0}(\Delta(A)) \rightarrow H_{0}\left(U_{3}\right) \oplus H_{0}\left(U_{4}\right) \rightarrow H_{0}\left(U_{2}\right)$ which gives the exact sequence $0 \rightarrow \mathbb{Z}^{4} \rightarrow H_{1}\left(U_{2}\right) \rightarrow \mathbb{Z}^{3} \rightarrow \mathbb{Z} \oplus \mathbb{Z} \rightarrow \mathbb{Z}$. It follows that $H_{1}\left(U_{2}\right)=\mathbb{Z}^{6}$. We obtain the nontrivial homology groups for $U_{2}$ :

$$
H_{i}\left(U_{2}\right)=\left\{\begin{array}{cl}
\mathbb{Z}^{3} \oplus \mathbb{Z}^{3} \oplus \mathbb{Z}, & i=2, \\
\mathbb{Z}^{6}, & i=1, \\
\mathbb{Z}, & i=0 .
\end{array}\right.
$$

We consider now the exact homology sequence of the pair $\left(U, U_{2}\right)$. It immediately gives that $H_{4}\left(U / U_{2}\right)=\mathbb{Z}$. We further obtain the exact sequence $0 \rightarrow H_{3}\left(U / U_{2}\right) \rightarrow H_{2}\left(U_{2}\right) \rightarrow H_{2}(U) \rightarrow$ $H_{2}\left(U / U_{2}\right) \rightarrow H_{1}\left(U_{2}\right) \rightarrow 0$, which gives the exact sequence $0 \rightarrow H_{3}\left(U / U_{2}\right) \rightarrow \mathbb{Z}^{7} \rightarrow \mathbb{Z}^{2} \rightarrow$ $H_{2}\left(U / U_{2}\right) \rightarrow \mathbb{Z}^{6} \rightarrow 0$. Note that the map $H_{2}\left(U_{2}\right) \rightarrow H_{2}(U)$ is induced by the inclusion $U_{2} \rightarrow U$. Let us consider a cell decomposition for $\mathbb{C} P^{1}=S^{2}$ which consists of the 2 two-dimensional cells $D_{1}, D_{2}$, then the 3 one-dimensional cells $I_{1}, I_{2}, I_{3}$ and the 3 zero-dimensional cells given by the points from $A$. Then the seven generators for $H_{2}\left(U_{2}\right)$ are given by $\left(D_{1} \cup D_{2}\right) \times A, A \times\left(D_{1} \cup D_{2}\right)$ and $\Delta\left(D_{1} \cup D_{2}\right)$, while the generators for $H_{2}(U)$ are given by $\left(D_{1} \cup D_{2}\right) \times\{*\}$ and $\{*\} \times\left(D_{1} \cup D_{2}\right)$, where $\{*\}$ is a fixed point from $A$. It implies that the map $H_{2}\left(U_{2}\right) \rightarrow H_{2}(U)$ is an epimorphism which implies that $H_{3}\left(U / U_{2}\right)=\mathbb{Z}^{5}$. The exact sequence $0 \rightarrow H_{2}\left(U / U_{2}\right) \rightarrow \mathbb{Z}^{6} \rightarrow 0$ implies that $H_{2}\left(U / U_{2}\right)=\mathbb{Z}^{6}$. Since all three spaces $U, U_{2}, U / U_{2}$ are connected we obtain that $H_{1}\left(U / U_{2}\right)=0$ and $H_{0}\left(U / U_{2}\right)=\mathbb{Z}$. Altogether the nontrivial homology groups for $U / U_{2}$ are:

$$
H_{i}\left(U / U_{2}\right)=\left\{\begin{array}{cc}
\mathbb{Z}, & i=4, \\
\mathbb{Z}^{5}, & i=3, \\
\mathbb{Z}^{6}, & i=2, \\
\mathbb{Z}, & i=0
\end{array}\right.
$$

Together with (47) this proves the statement.

Proof. We provide also the proof of the previous statement which is more geometrical. Let

$$
X=\Delta_{5,2} \times \mathbb{C} P^{1} \times \mathbb{C} P^{1}
$$

and

$$
Y=\left(\partial \Delta_{5,2} \times \mathbb{C} P^{1} \times \mathbb{C} P^{1}\right) \cup\left(\Delta_{5,2} \times A \times \mathbb{C} P^{1}\right) \cup\left(\Delta_{5,2} \times \mathbb{C} P^{1} \times A\right) .
$$

Then

$$
X / Y \cong\left(\stackrel{\circ}{\Delta}_{5,2} \times\left(\left(S^{2} \vee S^{1} \vee S^{1}\right) \backslash\{*\}\right) \times\left(\left(S^{2} \vee S^{1} \vee S^{1}\right) \backslash\{*\}\right)\right) \cup\{*\}
$$

It follows that

$$
X / Y \cong S^{8} \vee\left(\vee_{4} S^{7}\right) \vee\left(\vee_{4} S^{6}\right)
$$

Therefore,

$$
H_{8}(X / Y)=\mathbb{Z}, H_{7}(X / Y)=\mathbb{Z}_{54}^{4}, H_{6}(X / Y)=\mathbb{Z}^{4}, H_{0}(X / Y)=\mathbb{Z}
$$


Let $Z=\Delta_{5,2} \times \Delta\left(\mathbb{C} P^{1} \times \mathbb{C} P^{1}\right)$. Then $Z \cap Y=\Delta_{5,2} \times \Delta(A)$. It follows that

$$
Z /(Z \cap Y)=\left(\stackrel{\circ}{\Delta}_{5,2} \times \Delta\left(\mathbb{C} P_{A}^{1}\right)\right) \cup\{*\},
$$

that is

$$
Z /(Z \cap Y) \cong\left(\stackrel{\circ}{\Delta}_{5,2} \times\left(\left(S^{2} \vee S^{1} \vee S^{1}\right) \backslash\{*\}\right)\right) \cup\{*\}
$$

Therefore,

$$
H_{6}(Z /(Z \cap Y))=\mathbb{Z}, H_{5}(Z /(Z \cap Y))=\mathbb{Z}^{2}, H_{0}(Z /(Z \cap Y))=\mathbb{Z} .
$$

Set $C=Z /(Z \cap Y)$. Then $V_{3} / V_{2} \cong(X / Y) / C$. Using this we compute the homology groups for $V_{3} / V_{2}$. The exact homology sequence of the pair $(X / Y, C)$ immediately gives that

$$
H_{8}(X / U)=\mathbb{Z}, H_{0}(X / U)=0, H_{i}(X / U)=0, i=1,2,3,4,5 .
$$

We need to compute $H_{7}((X / Y) / C)$ and $H_{6}((X / Y) / C)$. Let us consider the exact sequence:

$$
\begin{aligned}
0=H_{7}(C) \rightarrow & \mathbb{Z}^{4}=H_{7}(X / Y) \rightarrow H_{7}((X / Y) / C) \rightarrow \mathbb{Z}=H_{6}(C) \rightarrow \mathbb{Z}^{4}=H_{6}(X / Y) \\
& \rightarrow H_{6}((X / Y) / C) \rightarrow \mathbb{Z}^{2}=H_{5}(C) \rightarrow 0=H_{5}(X / Y) .
\end{aligned}
$$

The map $\mathbb{Z}^{4}=H_{7}(X / Y) \rightarrow H_{7}((X / Y) / C)$ is a monomorphism. The map $\mathbb{Z}=H_{6}(C) \rightarrow$ $\mathbb{Z}^{4}=H_{6}(X / Y)$ is induced by the diagonal embedding $S^{2} \rightarrow\left(\left(S^{2} \backslash\{*\}\right) \times\left(S^{2} \backslash\{*\}\right)\right) \cup\{*\} \cong S^{4}$, so it is trivial. It follows from (52) that

$$
H_{7}((X / Y) / C)=\mathbb{Z}^{5} .
$$

It further implies that the map $\mathbb{Z}^{4}=H_{6}(X / Y) \rightarrow H_{6}((X / Y) / C)$ is a monomorphism. Since the map $H_{6}((X / Y) / C) \rightarrow \mathbb{Z}^{2}=H_{5}(C)$ is an epimorphism it follows that

$$
H_{6}((X / Y) / C)=\mathbb{Z}^{6} \text {. }
$$

The universal coefficient theorem implies:

Corollary 12.18. The nontrivial homology groups for $V_{3} / V_{2}$ with $\mathbb{Z}_{2}$-coefficients are as follows:

$$
H_{8}\left(V_{3} / V_{2} ; \mathbb{Z}_{2}\right) \cong \mathbb{Z}_{2}, H_{7}\left(V_{3} / V_{2} ; \mathbb{Z}_{2}\right) \cong \mathbb{Z}_{2}^{5}, H_{6}\left(V_{3} / V_{2} ; \mathbb{Z}_{2}\right) \cong \mathbb{Z}_{2}^{6}, H_{0}\left(V_{3} / V_{2} ; \mathbb{Z}_{2}\right) \cong \mathbb{Z}_{2}
$$

Combining Proposition 12.14 and Lemma 12.17 we obtain the homology groups for $G_{5,2} / T^{5}$ with $\mathbb{Z}_{2}$-coefficients.

Theorem 12.19. The non-trivial homology groups for $V_{3}=G_{5,2} / T^{5}$ with $\mathbb{Z}_{2}$-coefficients are

$$
H_{0}\left(V_{3} ; \mathbb{Z}_{2}\right)=H_{5}\left(V_{3}, \mathbb{Z}_{2}\right)=H_{6}\left(V_{3} ; \mathbb{Z}_{2}\right)=H_{8}\left(V_{3} ; \mathbb{Z}_{2}\right) \cong \mathbb{Z}_{2} .
$$

Proof. The exact homology sequence of the pair $\left(V_{3}, V_{2}\right)$ directly implies that $H_{8}\left(V_{3} ; \mathbb{Z}_{2}\right) \cong$ $H_{0}\left(V_{3} ; \mathbb{Z}_{2}\right) \cong \mathbb{Z}_{2}$ and $H_{i}\left(V_{3} ; \mathbb{Z}_{2}\right) \cong 0$ for $i \neq 5,6,7$.

We analyze the map $\mathbb{Z}_{2}^{5} \cong H_{7}\left(V_{32} ; \mathbb{Z}_{2}\right) \rightarrow H_{6}\left(V_{2} ; \mathbb{Z}_{2}\right) \cong \mathbb{Z}_{2}^{6}$. The generator from $H_{7}\left(V_{32}\right)$ which maps in (52) to the generator in $H_{6}(C)$, maps to an element $c_{\Delta}$ in $H_{6}\left(V_{2}\right)$ that contains $e_{45}$. Recall that the other generators in $H_{7}\left(V_{32}\right)$ are by the proof of Proposition 12.17 given by $m_{l}^{1}=\stackrel{\circ}{\Delta}_{5,2} \times\left(S^{2} \backslash\{*\}\right) \times\left(S_{l}^{1} \backslash\{*\}\right), m_{l}^{2}=\stackrel{\circ}{\Delta}_{5,2} \times\left(S_{l}^{1} \backslash\{*\}\right) \times\left(S^{2} \backslash\{*\}\right), l=1,2$. These are cells of the orbit space of the main stratum and their six-dimensional boundaries consist of the 6-cells of the other strata whose set of parameters is in the boundary of the set of parameters of the 3-cells 
$\left(S^{2} \backslash\{*\}\right) \times\left(S_{l}^{1} \backslash\{*\}\right)$ and $\left(S_{l}^{1} \backslash\{*\}\right) \times\left(S^{2} \backslash\{*\}\right)$ in $\tilde{\mathcal{F}}, l=1,2$. More precisely, since we want to consider the six-boundaries of these cells in $V_{3}$ we write them in the form

$$
m_{l}^{1}=\stackrel{\circ}{\Delta}_{5,2} \times F_{l}^{1}, \quad m_{l}^{2}=\stackrel{\circ}{\Delta}_{5,2} \times F_{l}^{2}, l=1,2,
$$

where

$$
\begin{aligned}
& F_{l}^{1}=\left\{\left(\left(c_{1}: c_{1}^{\prime}\right),\left(\phi_{2 l}: \phi_{2 l}^{\prime}\right),\left(c_{3}: c_{3}^{\prime}\right)\right), c_{1} \phi_{2}^{\prime} c_{3}=c_{1}^{\prime} \phi_{2} c_{3}^{\prime}\right\}, \\
& F_{l}^{2}=\left\{\left(\left(\phi_{1 l}: \phi_{1 l}^{\prime}\right),\left(c_{2}: c_{2}^{\prime}\right),\left(c_{3}: c_{3}^{\prime}\right)\right), \phi_{1} c_{2}^{\prime} c_{3}=\phi_{1}^{\prime} c_{2} c_{3}^{\prime}\right\},
\end{aligned}
$$

and $\left(\phi_{i 1}: \phi_{i 1}^{\prime}\right)$ is a path in $\mathbb{C} P_{A}^{1}$ which connects $(0: 1)$ and $(1: 1)$, while $\left(\phi_{i 2}: \phi_{i 2}^{\prime}\right)$ is a path in $\mathbb{C} P_{A}^{1}$ which connects $(1: 1)$ and $(1: 0)$ for $i=1,2$.

We compute the six-dimensional boundaries of these cells in $V_{3}$. Since we work with $\mathbb{Z}_{2^{-}}$ coefficients we do not need to take care about an orientation issue. Recall that the cells in $V_{2}$ of the form $\stackrel{\circ}{K}_{i j}(9) \times\left(S^{2} \backslash\{*\}\right)$ we denoted by $e_{i j}$. Thus, as the six-boundaries of the cells $m_{l}^{k}$, we obtain:

$$
\begin{array}{ll}
m_{1}^{1} \rightarrow e_{15}+e_{35}+e_{23}+e_{12}, & m_{2}^{1} \rightarrow e_{35}+e_{12}+e_{25}+e_{13}, \\
m_{1}^{2} \rightarrow e_{14}+e_{34}+e_{23}+e_{12}, & m_{2}^{2} \rightarrow e_{12}+e_{13}+e_{24}+e_{34} .
\end{array}
$$

Following notation from the proof of Proposition 12.14 these boundaries can be written as

$$
\begin{aligned}
m_{1}^{1} \rightarrow c_{12}+c_{23}, \quad m_{2}^{1} & \rightarrow c_{12}+c_{13}, \\
m_{1}^{2} \rightarrow c_{12}+c_{14}+c_{23}+c_{24}, \quad m_{2}^{2} & \rightarrow c_{12}+c_{13}+c_{24}+c_{34} .
\end{aligned}
$$

Comparing this with (46) we see that these elements together with $c_{\Delta}$ give five of the six generators in $H_{6}\left(V_{2}\right)$. The exact homology sequence implies that $H_{7}\left(V_{3} ; \mathbb{Z}_{2}\right)=0$.

The six-dimensional cycles in $V_{3}$ comes either from $V_{2}$ either from $V_{3} / V_{2}$. We have just proved that five of the six non-cohomologous 6 -cycles in $V_{2}$ are eliminated by the 7-cells in $V_{3}$, which implies that one cycle for $V_{2}$ survives after embedding in $H_{6}\left(V_{3}\right)$. The four generators in $H_{6}\left(V_{3} / V_{2}\right)$ correspond to the six-cells in $V_{3} / V_{2}$ of the form $p_{k l}=\stackrel{\circ}{\Delta}_{5,2} \times S_{l}^{1} \times S_{k}^{1}, k, l=1,2$. Their boundaries in $V_{3}$ belong to $V_{2}$ and they map trivially to $H_{5}(C)$, after restriction to $C$. In order to describe the boundaries of $p_{k l}$ explicitly we consider them in the form $p_{k l}=\stackrel{\circ}{\Delta}_{5,2} \times p_{l}^{k}$, where $p_{l}^{k}=\left\{\left(\left(\phi_{1 l}: \phi_{1 l}^{\prime}\right),\left(\phi_{2 k}: \phi_{2 k}^{\prime}\right),\left(\phi: \phi^{\prime}\right)\right) \in\left(\mathbb{C} P_{A}^{1}\right)^{3}, \phi_{1 l} \phi_{2 k}^{\prime} \phi=\phi_{1 l}^{\prime} \phi_{2 k} \phi^{\prime}\right\}$ and $\left(\phi_{i j}: \phi_{i j}^{\prime}\right)$ are as above. Therefore, following notation from the proof of Proposition 12.13, we obtain that these generators map to the following five-cycles in $V_{2}$ :

$$
\begin{aligned}
\stackrel{\circ}{\Delta} 5,2_{5} \times S_{1}^{1} \times S_{1}^{1} \rightarrow f_{14}^{1}+f_{23}^{1}+f_{23}^{2}+f_{15}^{1}+f_{34}^{1}+f_{35}^{1}+f_{12}^{1}+f_{12}^{2}, \\
\stackrel{\circ}{\Delta}_{5,2} \times S_{1}^{1} \times S_{2}^{1} \rightarrow f_{14}^{2}+f_{25}^{1}+f_{34}^{2}+f_{35}^{1}+f_{12}^{1}+f_{12}^{2}, \\
\stackrel{\circ}{\Delta}_{5,2} \times S_{2}^{1} \times S_{2}^{1} \rightarrow f_{34}^{2}+f_{35}^{2}+f_{12}^{1}+f_{12}^{2}+f_{13}^{1}+f_{13}^{2}+f_{24}^{2}+f_{25}^{2}, \\
\stackrel{\circ}{\Delta}_{5,2} \times S_{2}^{1} \times S_{1}^{1} \rightarrow f_{15}^{2}+f_{35}^{2}+f_{34}^{1}+f_{12}^{1}+f_{12}^{2}+f_{24}^{1} .
\end{aligned}
$$

These cycles represent four generators in $H_{5}\left(V_{2} ; \mathbb{Z}_{2}\right)=\mathbb{Z}_{2}^{7}$. The other two generators in $H_{6}\left(V_{3} / V_{2}\right)$ are by (52) the generators whose boundaries give the generators for $H_{5}(C)$. Let $c_{1}$ and $c_{2}$ be cycles in $V_{2}$ obtained as the boundaries of these generators for $H_{6}\left(V_{3} / V_{2}\right)=H_{6}((X / Y) / C)$, which after restriction to $C$ give the generators for $H_{5}(C)$, according to (51) and (53). Since the generators for $H_{5}(C)$ are given by the cells $\stackrel{\circ}{\Delta}_{5,2} \times \Delta\left(S_{l}^{1} \times S_{l}^{1}\right), l=1,2$ the cycle $c_{1}$ must $f_{45}^{1}$ as a summand and the cycle $c_{2}$ must contain $f_{45}^{2}$ as a summand, while $f_{45}^{1}+f_{45}^{2}$ can not be contained as 
a summand in both cycles. Therefore, $c_{1}, c_{2}$ are linearly independent and they are independent with the cycles obtained from $p_{k l}$, which implies that they eliminate two more generators in $H_{5}\left(V_{2} ; \mathbb{Z}_{2}\right)$. We obtain the exact homology sequence

$$
0 \rightarrow \mathbb{Z}_{2} \rightarrow H_{6}\left(V_{3} ; \mathbb{Z}_{2}\right) \rightarrow H_{6}\left(V_{3} / V_{2} ; \mathbb{Z}_{2}\right)=\mathbb{Z}_{2}^{6} \rightarrow H_{5}\left(V_{2} ; \mathbb{Z}_{2}\right)=\mathbb{Z}_{2}^{7}
$$

where the map $\mathbb{Z}_{2}^{6} \rightarrow \mathbb{Z}_{2}^{7}$ is a monomorphism. It implies that $H_{6}\left(V_{3}\right) \cong \mathbb{Z}_{2}$. We further obtain the exact sequence

$$
0 \rightarrow \mathbb{Z}_{2} \rightarrow H_{5}\left(V_{3} ; \mathbb{Z}_{2}\right) \rightarrow H_{5}\left(V_{3} / V_{2} ; \mathbb{Z}_{2}\right) \cong 0,
$$

which implies $H_{5}\left(V_{3} ; \mathbb{Z}_{2}\right) \cong \mathbb{Z}_{2}$.

Theorem 12.20. The non-trivial integral homology of the orbit space $V_{3}=G_{5,2} / T^{5}$ are given by $H_{0}\left(V_{3}\right)=H_{8}\left(V_{3}\right) \cong \mathbb{Z}$ and $H_{5}\left(V_{3}\right) \cong \mathbb{Z}_{2}$.

Proof. The universal coefficient formula immediately implies that $H_{i}\left(V_{3}\right)=0, i \neq 0,5,6,8$ and that $H_{0}\left(V_{3}\right)=H_{8}\left(V_{3}\right) \cong \mathbb{Z}$. Moreover, it implies that for $H_{5}\left(V_{3}\right)$ and $H_{6}\left(V_{3}\right)$ two possibilities may occur, that is either $H_{5}\left(V_{3}\right)=H_{6}\left(V_{3}\right) \cong \mathbb{Z}$ either $H_{5}\left(V_{3}\right) \cong \mathbb{Z}_{2}, H_{6}\left(V_{3}\right)=0$. Now an exact homology sequence of the pair $\left(V_{3}, V_{2}\right)$ produces an exact sequence

$$
\mathbb{Z}^{6} \cong H_{6}\left(V_{3} / V_{2}\right) \rightarrow H_{5}\left(V_{2}\right) \cong \mathbb{Z}^{6} \oplus \mathbb{Z}_{2} \rightarrow H_{5}\left(V_{3}\right)
$$

Note that the torsion element in $H_{5}\left(V_{2}\right)$ is represented by a cell of the form $\stackrel{\circ}{O}_{i} \times\left(S_{+}^{2} \cup S_{-}^{2}\right)$. On the other hand it follows from the proof of Theorem 12.19 that the six-dimensional cells in $V_{3} / V_{2}$ are either of the form $\stackrel{\circ}{\Delta}_{5,2} \times S_{l}^{1} \times S_{k}^{1}$ either the restriction of their 5-boundaries to $C$ is of the form $\stackrel{\circ}{\Delta}_{5,2} \times \Delta\left(S^{1} \times S^{1}\right)$. Therefore, the cells of the form $\stackrel{\circ}{O}_{i} \times\left(S_{+}^{2} \cup S_{-}^{2}\right)$ do not contribute in 5boundaries of the six-dimensional cells from $V_{3} / V_{2}$. It implies that an image of the homomorphism $H_{6}\left(V_{3} / V_{2}\right) \rightarrow H_{5}\left(V_{2}\right)$ does not contain the group $\mathbb{Z}_{2}$. It further implies that the group $H_{5}\left(V_{3}\right)$ has a torsion, that is $H_{5}\left(V_{3}\right) \cong \mathbb{Z}_{2}$, which completes the proof.

Remark 12.21. In the meantime motivated by our work the same result on homology groups for the orbit space $G_{5,2} / T^{5}$ is obtained in [25] using geometric invariant theory and in particular the result that geometric invariant theory quotients for the standard linearisation of $\left(\mathbb{C}^{*}\right)^{5}$-action on $G_{5,2}$ are the del Pezzo surfaces of degree 5. We are very greatfull to Hendrik Suess for pointing out to us his paper which helped us to correct some mistakes in previous calculations.

Corollary 12.22. The orbit space $G_{5,2} / T^{5}$ is a rational homology sphere $S^{8}$.

Corollary 12.23. The orbit space $G_{5,2} / T^{5}$ is homotopy equivalent to the space obtained by attaching the disc $D^{8}$ to the space $\Sigma^{4} \mathbb{R} P^{2}$.

Proof. It follows from Theorem 12.20 that the six-skeleton for $G_{5,2} / T^{5}$ is homotopy equivalent to the space obtained by attaching the disc $D^{6}$ to $S^{5}$ by a characteristic map $S^{5} \rightarrow S^{5}$ of degree 2, so the resulting space is a 4-suspension of $\mathbb{R} P^{2}$. Since $H_{7}\left(G_{5,2} / T^{5}\right)$ is trivial, it follows that $G_{5,2} / T^{5}$ is obtained by attaching the disc $D^{8}$ to the space $\Sigma^{4} \mathbb{R} P^{2}$.

Let us consider now one of the five equivariant embeddings $G_{4,2} \rightarrow G_{5,2}$ and let $X=\left(G_{5,2} / T^{5}\right) /\left(G_{4,2} / T^{4}\right)$. Since by [7], the space $G_{4,2} / T^{4}$ is homeomorphic to $S^{5}$, it follows that the space $X$ is homeomorphic to $\left(G_{5,2} / T^{5}\right) / S^{5}$. From homology computations for $G_{5,2} / T^{5}$ and, in particular, the proof of Proposition 12.14 we immediately obtain: 
Theorem 12.24. The nontrivial homology groups for $X$ are

$$
H_{0}(X)=H_{6}(X)=H_{8}(X) \cong \mathbb{Z} .
$$

Moreover, it holds

Corollary 12.25. The space $X$ is homotopy equivalent either to $S^{3} * \mathbb{C} P^{2}$ either to $S^{6} \vee S^{8}$.

Proof. From our description of the cell structure of the orbit space $G_{5,2} / T^{5}$ it follows that the 2skeleton of a quotient of $G_{5,2} / T^{5}$ by the sphere $S^{3}$ consists of one point, so the same holds for the space $X$. The description of the homology groups for $X$ and the Hurewitz theorem imply that the space $X$ is 5-connected, so it is homotopy equivalent to a $C W$-complex $C$ whose 6 skeleton is the sphere $S^{6}$. Moreover, since $H_{7}(C)=0$ and $H_{8}(C)=\mathbb{Z}$, it follows that $C$ is homotopy equivalent to a $C W$-space which is obtained by attaching the disc $D^{8}$ to the sphere $S^{6}$ by a characteristic map $f: S^{7} \rightarrow S^{6}$. The map $f$ is either homotopy equivalent to the trivial map or it is homotopy equivalent to the 4-suspension of the Hopf map $S^{3} \rightarrow S^{2}$. Therefore, in the first case $X$ is homotopy equivalent to the wedge $S^{6} \vee S^{8}$, while in the second case $X$ is homotopy equivalent to the 4 -suspension of $\mathbb{C} P^{2}$. The two cell complexes $S^{6} \vee S^{8}$ and the 4 -suspension of $\mathbb{C} P^{2}$ can be differentiated by the fact that in the first case the action of the Steenrod operation $S_{q}^{2}$ is trivial, while in the second case it is non-trivial.

We determine an exact homotopy type for $X$.

Theorem 12.26. The space $X=\left(G_{5,2} / T^{5}\right) / S^{5}$ is homotopy equivalent to $S^{3} * \mathbb{C} P^{2}$.

Proof. The spaces $X$ and $\partial \Delta_{5,2} * \mathbb{C} P^{2}$ are simply-connected $\mathrm{CW}$-spaces and have the same integral homology groups. We will prove that there exists a continuous map $h_{5}: X \rightarrow \partial \Delta_{5,2} * \mathbb{C} P^{2}$ which induces an isomorphism in their homology groups and then, by the Whitehead theorem, it will follow that the map $h_{5}$ is homotopy equivalence.

Since the homology groups for $X$ are trivial in the dimensions less or equal then 5 , it follows that the 5 -skeleton $C$ of this space is contractible which implies that $X / C$ is homotopy equivalent to $X$. From the description of a cell decomposition of $G_{5,2} / T^{5}$, it follows that the orbit spaces of the strata, whose admissible polytopes are different from $\Delta_{5,2}$ and $K_{i j}(9)$, contain only the cells of the dimension less or equal then 5 . Thus, $X / C$ contains only cells from the orbit spaces of the strata whose admissible polytopes are $\Delta_{5,2}$ or $K_{i j}(9)$. Recall that $W / T^{5} \cong_{\Delta_{5,2}}^{\circ} \times F \cong \stackrel{\circ}{\Delta, 2}_{5} \times \tilde{F}_{12}$ and $W_{i j}(9) / T^{5} \cong \stackrel{\circ}{K}_{i j}(9) \times \mathbb{C} P_{A}^{1} \stackrel{\circ}{K_{K}} i j(9) \times \tilde{F}_{i j, 12}$, where by $\tilde{F}_{12}$ and $\tilde{F}_{i j, 12}$ we denote, as previously, the virtual spaces of parameters for these strata in the chart $M_{12}$. Note that virtual spaces of parameters are the subspaces in $\tilde{\mathcal{F}}$.

Let $Z=X \backslash C$. It is defined the map

$$
h_{5}^{1}: Z \rightarrow \Delta_{5,2} \times \tilde{\mathcal{F}},
$$

by the projection on $\Delta_{5,2}$ and by an embedding into $\tilde{\mathcal{F}}$. Note that the image of $Z$ by $h_{5}^{1}$ belongs to $\stackrel{\circ}{\Delta}_{5,2} \times \tilde{\mathcal{F}}$. Moreover, the virtual spaces of parameters $\tilde{F}_{12}$ and $\tilde{F}_{i j, 12}$ are open sets and their union is not the whole $\tilde{\mathcal{F}}$, which implies that the image of $Z$ is not the whole $\tilde{\mathcal{F}}$. Further, it follows from Corollary 7.11 that $\tilde{\mathcal{F}}$ is homeomorphic to a space $Y$ which is the blowup of $\mathbb{C} P^{2}$ at four points. We consider the map

$$
h_{5}^{2}: \Delta_{5,2} \times \tilde{\mathcal{F}} \rightarrow \Delta_{5,2} \times \mathbb{C} P^{2},
$$


defined by the blowdown of $\tilde{\mathcal{F}}$ at these four points. Next, we consider the projection

$$
h_{5}^{3}: \Delta_{5,2} \times \mathbb{C} P^{2} \rightarrow \partial \Delta_{5,2} * \mathbb{C} P^{2} .
$$

We obtain the map

$$
h_{5}^{3} \circ h_{5}^{2} \circ h_{5}^{1}: Z \rightarrow \partial \Delta_{5,2} * \mathbb{C} P^{2} .
$$

Denote by $D$ the 5 -skeleton of the space $\partial \Delta_{5,2} * \mathbb{C} P^{2} \cong\left(\Delta_{5,2} \times \mathbb{C} P^{2}\right) /\left(\partial \Delta_{5,2} \times \mathbb{C} P^{2}\right)$. Then $D$ is contractible and $\left(\partial \Delta_{5,2} * \mathbb{C} P^{2}\right) / D$ is homotopy equivalent to $\partial \Delta_{5,2} * \mathbb{C} P^{2}$. The space $D$ contains the cell $\stackrel{\circ}{\Delta}_{5,2} \times\{*\}$, but since $h_{5}^{1}(Z)$ is a strict subset of $\tilde{\mathcal{F}}$ we may always choose $\{*\} \in \mathbb{C} P^{2}$ such that $h_{5}^{3} \circ h_{5}^{2} \circ h_{5}^{1}(Y) \subset\left(\partial \Delta_{5,2} * \mathbb{C} P^{2}\right) \backslash D$.

In this way we obtain the map

$$
h_{5}: X \cong X / C \rightarrow\left(\partial \Delta_{5,2} * \mathbb{C} P^{2}\right) / D \cong \partial \Delta_{5,2} * \mathbb{C} P^{2},
$$

which is defined by (59) on $X \backslash C$ and $h_{5}(C)=D$.

We prove that the map $h_{5}$ induces an isomorphism in integral homology groups. Since the spaces $X$ and $S^{3} * \mathbb{C} P^{2}$ have the nontrivial integral homology groups in degrees $0,6,8$, it is enough to prove that $h_{5}$ induces an isomorphism of the homology groups in degree 6. From calculations of the homology groups for $G_{5,2} / T^{5}$ it follows that the generator for $H_{6}(X)$ comes from $V_{2}$. More precisely, it follows from (56), (57) and (46) that for a generator in $H_{6}(X)$ one can take the cycle $c_{12}=e_{12}+e_{15}+e_{25}$. Recall that $e_{i j}$ are, by (41) and Remark 12.11, the six-dimensional cycles in $K_{i j}^{*}(9)$ and they are of the form $\stackrel{\circ}{K}_{i j}(9) \times \tilde{F}_{12, i j}^{\prime}$, where $\tilde{F}_{i j, 12}^{\prime} \subset \tilde{F}_{i j, 12}$ and $\tilde{F}_{i j, 12}^{\prime} \cong S^{2} \backslash\{*\}$. It follows from Theorem 9.14 that the virtual spaces of parameters $\tilde{F}_{24,12}, \tilde{F}_{25,12}, \tilde{F}_{23,12}$ and $\tilde{F}_{12,12}$ after embedding them into the space $Y$ map to the blowups of $\mathbb{C} P^{2}$ at four points. It implies that after blowing down $Y$ at these four points these spaces map to the four points. The other spaces of parameters $\tilde{F}_{i j, 12}$ are not affected by this blowing down. Consequently, in the six-dimensional homology the image of the generator $c_{12}$ of $H_{6}\left(G_{5,2} / T^{5}\right)$ is given by the image of $e_{15}$. It holds that $\tilde{F}_{15,12}=\left(\left(c_{1}: c_{1}^{\prime}\right),(0: 1),(0: 1)\right) \subset \tilde{\mathcal{F}}$, which is equal to $\left(\left(c_{1}: c_{1}^{\prime}\right),(1: 0)\right) \in \mathbb{C} P^{1} \times \mathbb{C} P^{1}$ and this is further equal to $\left(c_{1}: 0: c_{1}^{\prime}\right) \in \mathbb{C} P^{2}$. It follows that the image of $e_{15}$ by the map $h_{5}$ is $\Delta_{5,2}^{\circ} \times\left(c_{1}: 0: c_{1}^{\prime}\right)$, which represents a generator for $H_{6}\left(\partial \Delta_{5,2} * \mathbb{C} P^{2}\right)$. This proves the statement.

From Theorem 3.17 and Example 3.19 it follows the result.

Corollary 12.27. The space $\Sigma\left(S_{5,2} / T^{5}\right)$ is homotopy equivalent to $S^{3} * \mathbb{C} P^{2}$.

These results enable us also to describe the module structure of $H^{*}\left(G_{5,2} / T^{5} ; \mathbb{Z}_{2}\right)$ over the $\bmod$ 2 Steenrod algebra as well.

Corollary 12.28. The Steenrod square $S q^{i}$ acts non-trivially on $H^{*}\left(G_{5,2} / T^{5} ; \mathbb{Z}_{2}\right)$ if and only if $i=1,2$.

Proof. It follows from the universal coefficient formula that the cohomology groups for $G_{5,2} / T^{5}$ with $\mathbb{Z}_{2}$-coefficients coincide with its $\mathbb{Z}_{2}$-homology groups. It immediately implies that $S q^{i}=0$ for $i \neq 1,2$. Moreover, being stable operation we have that $S q^{1}: H_{5}\left(V_{3} ; \mathbb{Z}_{2}\right) \rightarrow H_{6}\left(V_{3} ; \mathbb{Z}_{2}\right)$ coincides with $S q^{1}: H^{1}\left(\mathbb{R} P^{2} ; \mathbb{Z}_{2}\right) \rightarrow H^{2}\left(\mathbb{R} P^{2} ; \mathbb{Z}_{2}\right)$. The later one is defined by squaring, so it is nontrivial. In the same way $S q^{2}: H^{6}\left(V_{3} ; \mathbb{Z}_{2}\right) \rightarrow H^{8}\left(V_{3} ; \mathbb{Z}_{2}\right)$ coincides with $S q^{2}: H^{2}\left(\mathbb{C P}^{2} ; \mathbb{Z}_{2}\right) \rightarrow$ $H^{4}\left(\mathbb{C} P^{2} ; \mathbb{Z}_{2}\right)$ which is given by squaring, so it is non-trivial. 
Altogether this leads to the homotopy description of the orbit space $G_{5,2} / T^{5}$.

Theorem 12.29. The orbit space $G_{5,2} / T^{5}$ is homotopy equivalent to the space $X$ obtained by attaching the disc $D^{8}$ to the space $\Sigma^{4} \mathbb{R} P^{2}$ by the generator of the group $\pi_{7}\left(\Sigma^{4} \mathbb{R} P^{2}\right)$.

Proof. Since by [28] it holds $\pi_{7}\left(\Sigma^{4} \mathbb{R} P^{2}\right)=\mathbb{Z}_{4}$ we need to show that $G_{5,2} / T^{5}$ can not be obtained by attaching the disc $D^{8}$ to the space $\Sigma^{4} \mathbb{R} P^{2}$ by the map $f: S^{7} \rightarrow \Sigma^{4} \mathbb{R} P^{2}$ of order 2 . In order to verify this we first consider the exact homotopy sequence of the pair $\left(\Sigma^{4} \mathbb{R} P^{2}, S^{5}\right)$. Note that, as a consequence of the Blakers-Massey theorem, we have that $\pi_{i}\left(\Sigma^{4} \mathbb{R} P^{2}\right)=\pi_{i}\left(\Sigma^{4} \mathbb{R} P^{2} / S^{5}\right)$ for $i \leq 7$, since $\Sigma^{4} \mathbb{R} P^{2}$ is 4 -connected and the inclusion $S^{5} \rightarrow \Sigma^{4} \mathbb{R} P^{2}$ induces an isomorphism in homotopy groups up to degree 5 . Thus, since $\left(\Sigma^{4} \mathbb{R} P^{2}\right) / S^{5}$ is homotopy equivalent to $S^{6}$, the corresponding homotopy sequence writes as

$$
\mathbb{Z}_{2} \rightarrow \pi_{7}\left(\Sigma^{4} \mathbb{R} P^{2}\right) \cong \mathbb{Z}_{4} \rightarrow \mathbb{Z}_{2} \rightarrow \mathbb{Z}_{2} \rightarrow \pi_{6}\left(\Sigma^{4} \mathbb{R} P^{2}\right) \cong \mathbb{Z}_{2} \rightarrow \mathbb{Z}
$$

which gives an exact sequence

$$
\mathbb{Z}_{2} \rightarrow \mathbb{Z}_{4} \rightarrow \mathbb{Z}_{2} \rightarrow 0
$$

It follows that the element of order 2 in $\pi_{7}\left(\Sigma^{4} \mathbb{R} P^{2}\right)$ is coming from $\pi_{7}\left(S^{5}\right)$. Now, consider a map $f: S^{7} \rightarrow \Sigma^{4} \mathbb{R} P^{2}$. It induces the map $g: S^{7} \rightarrow S^{6}$ given by the composition $S^{7} \stackrel{f}{\rightarrow}$ $\Sigma^{4} \mathbb{R} P^{2} \rightarrow \Sigma^{4} \mathbb{R} P^{2} / S^{5} \cong S^{6}$. It follows from Theorem 12.26 and Corollary 12.25 that the map $g$ is a fourfold suspension of the Hopf map $S^{3} \rightarrow S^{2}$ and $G_{5,2} / T^{5}$ is homotopy equivalent to $S^{3} * \mathbb{C} P^{2}$. If the attaching map $f$ decomposes through a map $S^{7} \rightarrow S^{5} \stackrel{i}{\rightarrow} \Sigma^{4} \mathbb{R} P^{2}$, it would imply that $\left(G_{5,2} / T^{5}\right) / S^{5}$ is homotopy equivalent to $S^{8} \vee S^{6}$ which is not the case.

Let $\xi_{n, k}$ and $\eta_{n, n-k}$ be the canonical vector bundles over the Grassmannian $G_{n, k}$ and $X_{n, k}=$ $T\left(\xi_{n-1, k}\right), Y_{n, k}=T\left(\eta_{n-1, n-k}\right)$ - the corresponding Thom spaces equipped with the $T^{n}$-action. The problem of the description of the orbit space $G_{n, k} / T^{n}$ naturally brings the problem of the description of the orbit spaces for these Thom spaces. In the case $n=5$ and $k=2$, Corollary 2.30 and Corollary 2.31 imply:

Theorem 12.30. The orbit spaces $X_{5,2} / T^{5}$ is homotopy equivalent to $D^{8} \cup_{f} \Sigma^{4} \mathbb{R} P^{2}$ for the generator $f \in \pi_{7}\left(\Sigma^{4} \mathbb{R} P^{2}\right)$, while the orbit space $Y_{5,2} / T^{5}$ is homotopy equivalent to $S^{3} * \mathbb{C} P^{2}$.

\section{REFERENCES}

1. M. F. Atiyah, Convexity and commuting Hamiltonians, Bull. London Math. Soc. 14, no.1, 1982, 1-15.

2. Anton A. Ayzenberg, Torus actions of complexity 1 and their local properties, Proc. Steklov Inst. Math. 302, no.1, (2018), 16-32.

3. Anton Ayzenberg, Torus action on quaternionic projective plane and related spaces, arXiv:1903.03460.

4. Victor M. Buchstaber (joint with Svjetlana Terzić), (2n, k)-manifolds and applications, Mathematisches Forschung Institut Oberwolfach, Report No. 27/2014, p. 58, DOI: 10.4171/OWR/2014/27

5. Victor M. Buchstaber and Taras E. Panov, Torus Actions and Their Applications in Topology and Combinatorics, University Lectures Series, Vol. 24, American Mathematical Society, 2002.

6. Victor M. Buchstaber and Taras E. Panov, Toric topology, Mathematical Surveys and Monographs Vol. 204, American Mathematical Society, 2015.

7. Victor M. Buchstaber and Svjetlana Terzić, Topology and geometry of the canonical action of $T^{4}$ on the complex Grassmannian $G_{4,2}$ and the complex projective space $\mathbb{C} P^{5}$, Moscow Math. Jour. Vol. 16, Issue 2, (2016), 237273.

8. V. M. Buchstaber and S. Terzić, The foundations of $(2 n ; k)$-manifolds, Mat. Sbornik, Vol. 210, no.4, (2019), 41-86, (in Russian).

9. William Fulton, Introduction to Toric Varieties, Princeton University Press, 1993. 
10. V. Guillemin and V. Sternberg, Convexity properties of the moment mapping, Invent. Math. 67, $1982,491-513$.

11. I. M. Gelfand and R. MacPherson, Geometry in Grassmannians and a generalization of the dilogarithm, Adv. in Math. 44, no.3, (1982), 279-312.

12. I. M. Gelfand and V. V. Serganova, Combinatorial geometries and torus strata on homogeneous compact manifolds, Russ. Math. Surveys 42, no.2, (1987), 133-168.

13. I. M. Gelfand, R. M. Goresky, R. D. MacPherson and V. V. Serganova, Combinatorial Geometries, Convex Polyhedra, and Schubert Cells, Adv. in Math. 63, (1987), 301-316.

14. S. G. Hoggar, On KO theory of Grassmannians, Quart. J. Math. Oxford Ser. (2) 20, (1969), 447-463.

15. M. M. Kapranov, Chow quotients of Grassmannians I, I. M. Gel'fand Seminar, Adv. in Soviet Math., 16, part 2, Amer. Math. Soc. (1993), 29-110.

16. Yael Karshon and Susan Tolman, Classification of Hamiltonian torus actions with two-dimensional quotients, Geom. Topol. 18, no.2, (2014), 669-716.

17. Yael Karshon and Susan Tolman, Topology of complexity one quotients, arXiv:1810.01026.

18. S. Keel, Intersection theory of moduli space of stable n-pointed curves of genus zero, Trans. Amer. Math. Soc, 330, no. 2, (1992), 545-574.

19. Sean Keel and Jenia Tevelev, Geometry of Chow quotients of Grassmannians, Duke Math. J. 134, no. 2 (2006), 259-311.

20. Frances Clare Kirwan, Cohomology of Quotients in Symplectic and Algebraic Geometry, Mathematical Notes, 31, Princeton University Press, 1984.

21. Nikita Klemyatin, Universal spaces of parameters for complex Grassmann manifolds $G_{q+1,2}$, arXiv:1905.03047

22. Masashi Noji, Kazuaki Ogiwara, The smooth torus orbit closures in the Grassmannians, arXiv:1812.11472.

23. D. A. Timashev, Classification of G-manifolds of complexity 1, (Russian) Izv. Ross. Akad. Nauk Ser. Mat. 61, no.2 (1997), 127-162, translation in Izv. Math. 61, no.2 (1997), 363-397.

24. Hendrik Süss, Orbit spaces of maximal torus actions on oriented Grassmannians of planes, arXiv:1810.00981.

25. Hendrik Süss, Toric topology of the Grassmannian of planes in $\mathbb{C}^{5}$ and the del Pezzo surface of degree 5 , arXiv:1904.13301

26. Dmitri Timashev, Torus actions of complexity one, Toric topology, 349-364, Contemp. Math., 460, Amer. Math. Soc., Providence, RI, 2008.

27. G. Ziegler, Lectures on polytopes, Graduate Texts in Mathematics, 152. Springer-Verlag, New York, 1995.

28. Jie Wu, Homotopy Theory of the Suspensions of the Projective Plane, Memoirs of the AMS, 162, no. 769 (2003).

Steklov Mathematical Institute of the Russian Academy of Sciences, Moscow State UniverSity V. M. Lomonosov, Skolkovo Institute of Science And Technology, Moscow, Russia

E-mail address: buchstab@mi .ras.ru

Faculty of Science And Mathematics, University of Montenegro, Podgorica, Montenegro

E-mail address: sterzic@ucg.ac.me 